\title{
Comportamento assintótico de um problema parabólico não linear com termos concentrados na fronteira
}

\author{
Lucas Galhego Mendonça
}

\author{
DisSERTAÇÃO APRESENTADA \\ $\mathrm{AO}$ \\ Instituto DE MATEMÁticA E Estatística \\ DA \\ Universidade DE SÃo PAUlo \\ PARA \\ OBTENÇÃO DO TÍTULO \\ DE \\ Mestre EM CiÊnCIAS \\ Programa: Matemática Aplicada \\ Orientador: $\operatorname{Prof}^{\mathrm{a}}$. Dr ${ }^{\mathrm{a}}$. Gleiciane da Silva Aragão
}

Durante o desenvolvimento deste trabalho o autor recebeu auxílio financeiro da CAPES

São Paulo, março de 2018 


\section{Comportamento assintótico de um problema parabólico não linear com termos concentrados na fronteira}

Esta versão da dissertação contém as correções e alterações sugeridas pela Comissão Julgadora durante a defesa da versão original do trabalho, realizada em 01/03/2018. Uma cópia da versão original está disponível no Instituto de Matemática e Estatística da Universidade de São Paulo.

Comissão Julgadora:

- Prof ${ }^{\mathrm{a}}$. Dra ${ }^{\mathrm{a}}$. Gleiciane da Silva Aragão - IME - USP

- Prof. Dr. Flank David Morais Bezerra - UFPB

- Prof ${ }^{\mathrm{a}}$. Dra ${ }^{\mathrm{a}}$. Juliana Fernandes da Silva Pimentel - UFABC 


\section{Agradecimentos}

Primeiramente, agradeço a todos que passaram na minha vida durante essa etapa, pois contribuiram de alguma forma no meu amadurecimento para concluir o mestrado.

Em particular, gostaria de agradecer à minha família que compreendeu minha ausência e me auxiliou no percurso, em especial, à minha mãe, Vilma, por todas as suas contribuições a minha vida pessoal e acadêmica, por todo carinho e exemplo; à minha namorada, Laiana, por todo carinho, compreensão e companheirismo.

Agradeço à minha orientadora, professora Gleiciane, que me instigou e mostrou o caminho da Matemática e, ao longo desses anos, sempre me apoiou com paciência e dedicação.

Também agradeço aos meus amigos, em especial, à Gabriela e Danilo, que fazem da distância um detalhe.

Por fim, agradeço a todos os professores, os funcionários, o IME-USP, a UNIFESP-Diadema e a CAPES que me auxiliaram na caminhada. 


\section{Resumo}

\section{Mendonça, L.G. Comportamento assintótico de um problema parabólico não linear com}

termos concentrados na fronteira. Dissertação (Mestrado) - Instituto de Matemática e Estatística, Universidade de São Paulo, São Paulo, 2017.

Neste trabalho estudamos o comportamento assintótico das soluções de um problema parabólico não linear com condições de fronteira de Neumann homogêneas e com termos concentrados em uma vizinhança da fronteira, que contrai-se a fronteira quando um parâmetro tende à zero. Sob certas hipóteses de crescimento crítico das não linearidades, de sinal e dissipação, provamos que as soluções existem, são únicas e convergem, num determinado espaço de Sobolev, para a única solução de um problema parabólico não linear com condições de fronteira de Neumann não lineares. Provamos também a existência de atratores globais e que a família de atratores globais é semicontínua superiormente. Finalmente, concluímos a semicontinuidade superior da família de equilíbrios.

Palavras-chave: problema parabólico não linear, termos concentrados, comportamento assintótico, convergência das soluções, atratores, equilíbrios, semicontinuidade superior. 


\section{Abstract}

Mendonça, L.G. Asymptotic behavior of a nonlinear parabolic problem with terms concentrated in the boundary. Dissertação (Mestrado) - Instituto de Matemática e Estatística, Universidade de São Paulo, São Paulo, 2017.

In this work we study the asymptotic behavior of the solutions of a nonlinear parabolic problem with homogeneous Neumann boundary conditions and with terms concentrating in a neighborhood of the boundary, which shrinks to boundary as a parameter goes to zero. Under certain conditions of critical growth of the nonlinearities, of sign and dissipativeness, we prove that the solutions exists, are unique and converge, in a given Sobolev space, to the unique solution of a nonlinear parabolic problem with nonlinear Neumann boundary conditions. We also prove the existence of global attractors and that the family of global attractors is upper semicontinuous. Finally, we concluded the upper semicontinuity of the family of equilibria.

Keywords: nonlinear parabolic problems, concentrated terms, asymptotic behavior, convergence of solutions, attractors, equilibria, upper semicontinuity. 


\section{Sumário}

$\begin{array}{ll}\text { Lista de símbolos } & \text { ix }\end{array}$

1 Introdução $\quad \mathbf{1}$

1.1 Contextualização histórica e motivações . . . . . . . . . . . . . . . . 1

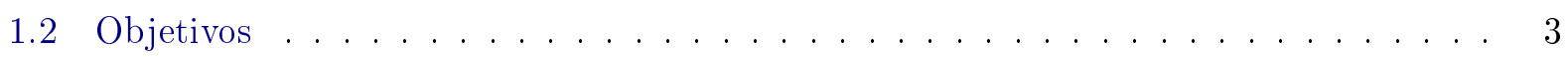

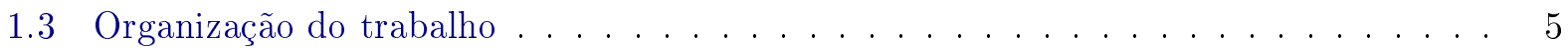

2 Notações e preliminares $\quad 7$

2.1 Operadores lineares . . . . . . . . . . . . . . . . . . . 7

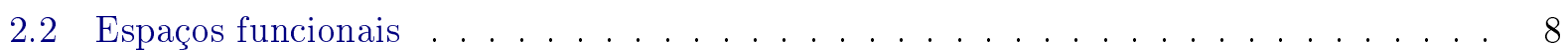

2.2 .1 O espaço das funções contínuas, $C^{0}(\Omega) \ldots \ldots \ldots \ldots \ldots$

2.2.2 O espaço das funções Hölder contínuas, $C^{0, l}(\Omega) \ldots \ldots \ldots \ldots$

2.2.3 O espaço das funções de classe $m$-diferenciáveis, $C^{m}(\Omega) \ldots \ldots \ldots$

2.2.4 O espaço das funções infinitamente diferenciáveis, $C^{\infty}(\Omega) \ldots \ldots \ldots \ldots$

2.2.5 O espaço das funções $p$-Lebesgue integráveis, $L^{p}(\Omega) \ldots \ldots \ldots$

2.2 .6 O espaço das distribuições, $\mathcal{D}^{\prime}(\Omega) \ldots \ldots \ldots \ldots \ldots \ldots$

2.2 .7 O espaço de Schwartz, $\mathcal{S}\left(\mathbb{R}^{n}\right) \ldots \ldots \ldots \ldots \ldots \ldots$

2.2 .8 Transformada de Fourier . . . . . . . . . . . . . . . . . . . . . 11

2.2 .9 Espaços de Sobolev em $\mathbb{R}^{n}$ e $\Omega \subset \mathbb{R}^{n}$ aberto . . . . . . . . . . . . . . 11

2.2.10 Espaços Potencial de Bessel em $\mathbb{R}^{n}$ e $\Omega \subset \mathbb{R}^{n}$ aberto . . . . . . . . . . . 13

2.2 .11 O operador traço em $H_{p}^{s}(\Omega) \ldots \ldots \ldots \ldots \ldots \ldots \ldots$

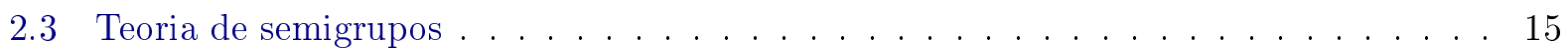

2.4 Escalas de interpolação e extrapolação . . . . . . . . . . . . . . . . . 18

2.5 Existência e unicidade de soluções de equações parabólicas . . . . . . . . . . . . . 20

2.6 Teoria de atratores . . . . . . . . . . . . . . . . . . . 22

2.7 Integrais concentradas . . . . . . . . . . . . . . . . . 24

2.8 Problemas elípticos com concentração . . . . . . . . . . . . . . 28

3 Existência, unicidade e limitação uniforme das soluções $\quad 31$

3.1 Formulação abstrata dos problemas parabólicos . . . . . . . . . . . . . . . . . . 32

3.2 Existência e unicidade de soluções locais . . . . . . . . . . . . . . . 36

3.3 Existência global e limitação uniforme de soluções . . . . . . . . . . . . . . . . . . . . 43 
4 Semicontinuidade superior dos atratores e dos equilíbrios $\quad 55$

4.1 Convergência das soluções . . . . . . . . . . . . . . . . . . . 55

4.2 Existência e semicontinuidade superior dos atratores globais . . . . . . . . . . . . 61

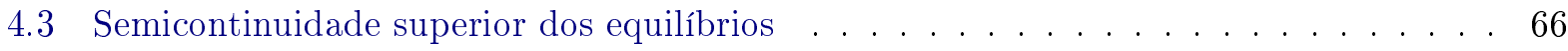

4.3.1 Formulação abstrata dos problemas elípticos . . . . . . . . . . . . . . 66

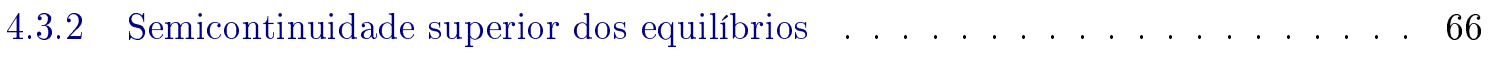

4.4 Considerações finais . . . . . . . . . . . . . . . . . . 67

$\begin{array}{ll}\text { A Outra abordagem para provar a condição de Lipschitz } & 69\end{array}$

$\begin{array}{ll}\text { Referências Bibliográficas } & 75\end{array}$ 


\section{Lista de símbolos}

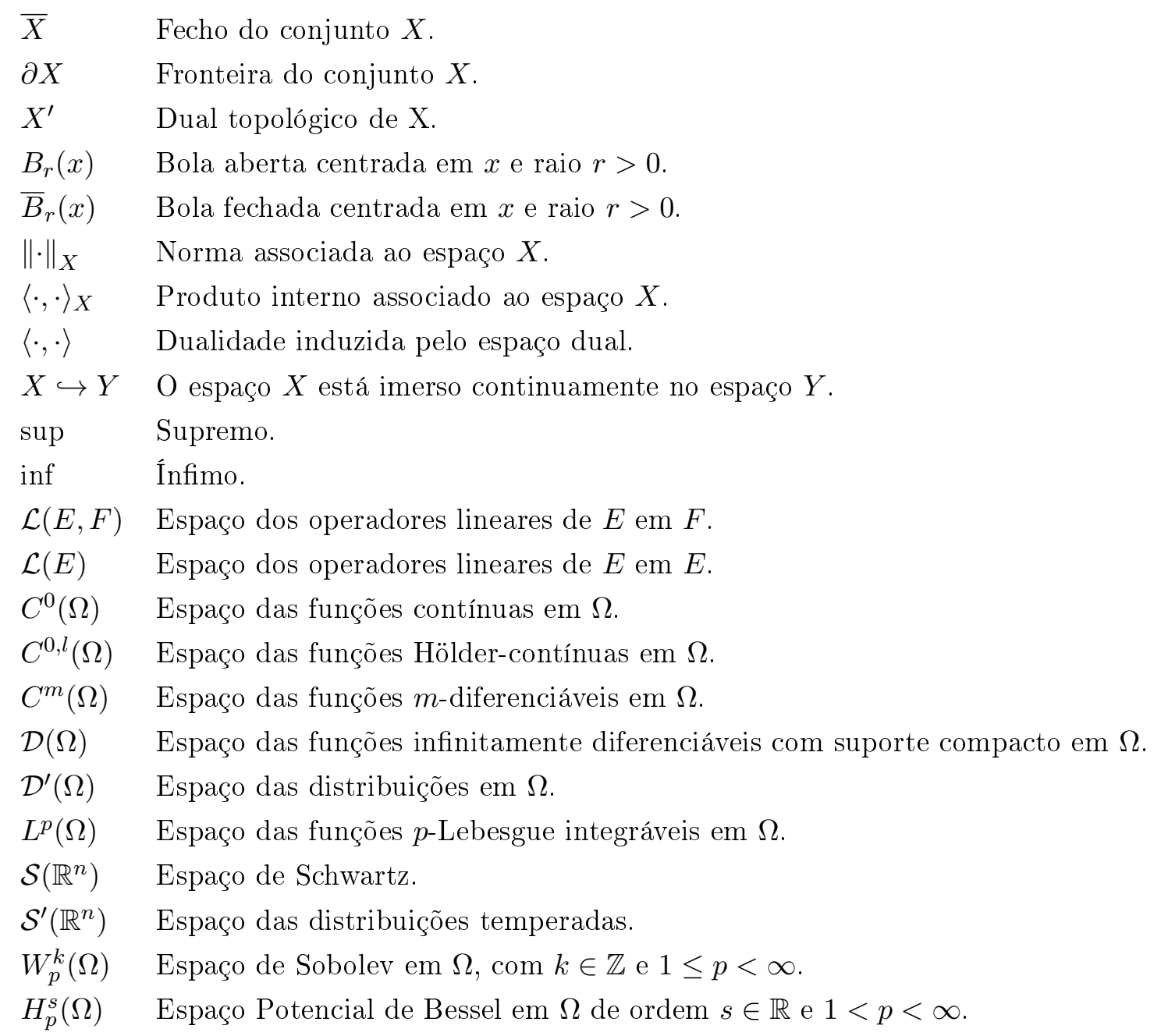




\section{Capítulo 1}

\section{Introdução}

\subsection{Contextualização histórica e motivações}

As Equações Diferenciais é uma área da Matemática que surgiu junto com o Cálculo e suas aplicações nas Ciências Naturais, principalmente, na Mecânica dos Corpos, enquanto, suas subáreas de pesquisa, como a Teoria Geométrica de Equações Diferenciais, Sistemas Dinâmicos, Equações Diferenciais Parciais e Funcionais (E.D.P. e E.D.F.) e outras, desenvolvem até hoje diferentes técnicas e abordagens para estudar seus diversos problemas.

As Equações Diferenciais são amplamente utilizadas como ferramenta para a modelagem matemática de fenômenos em diversas áreas de estudo, como Engenharia, Química, Biologia, Economia, entre outras. Entender o comportamento das equações e suas soluções pode ajudar a compreender melhor os modelos e, consequentemente, as situações modeladas.

Em geral, resolver um problema de Equações Diferenciais consiste em exibir uma solução analítica, como por exemplo uma função, em termo das funções elementares, que resolve as condições de fronteiras, as condições iniciais e a equação diferencial do problema. Contudo, nem sempre é possível exibir a fórmula fechada da solução, e por isso, uma outra abordagem é necessária: a teoria qualitativa das Equações Diferenciais. Como o próprio nome sugere, com a teoria qualitativa é possível fazer certas afirmações em relação às propriedades dessa solução, como o comportamento assintótico, isto é, saber qual o comportamento da solução em tempos grandes. Assim, apesar de não ser possível exibir a solução do problema, ainda é possível responder algumas perguntas e inferir previsões.

A teoria qualitativa obteve um grande avanço com os estudos de Henry Poincaré e outros matemáticos em meados do século XIX, aproximadamente em 1800, e no Século XX, a teoria das Equações Funcionais e Sistemas Dinâmicos obtiveram grandes avanços, principalmente com os trabalhos de Jack Hale.

Apesar de jovem, a teoria qualitativa das Equações Diferenciais Parciais está bem estruturada e subsidiada pelos resultados da Análise Funcional e Teoria de Operadores, das quais, dentro das diversas técnicas, utilizaremos a teoria de semigrupo (ver Carvalho (2012, 2001); Henry (1981) e Yosida (1980))para estudar o comportamento qualitativo das soluções de um tipo de Equação Diferencial Parcial parabólica não linear com termos concentrados numa vizinhança da fronteira do domínio e determinadas condições de contorno.

A teoria de Equações Diferenciais Parciais parabólicas vista do ponto da teoria de semigrupos, apesar de ser recente, possui diversos resultados quando olhamos para os problemas como uma equação de evolução em um espaço de dimensão infinita, em específico, de Banach, isto é, reescrevemos de alguma forma o problema parabólico original com uma equação que faz sentido em algum espaço de Banach, na qual, futuramente, denominaremos de formulação abstrata. Dentre os diversos resultados, vale ressaltar novamente as principais bibliografias sobre este assunto usadas neste trabalho Amann (1995); Carvalho (2012, 2001); Czaja (2002); Hale (1988); Haroske e Triebel (2008); Henry (1981); Temam (1988); Triebel (1978) e Yagi (2010).

As E.D.P's parabólicas não lineares podem também possuir condições de fronteira de Neumann 
ou Robin não lineares e, neste caso, precisamos assegurar sob quais condições nas não linearidades os problemas abstratos associados estão bem postos, isto é, existe uma única solução do problema que depende continuamente do dado inicial. Em Carvalho et al. (1997), Arrieta e Carvalho (1999) e Arrieta et al. (2000) os autores assumem condições de crescimento nas não linearidades para o problema estar bem posto no espaço de Sobolev $W_{p}^{1}(\Omega), 1<p<\infty$ e $\Omega \subset \mathbb{R}^{n}$, com $n \geq 1$. Por outro lado, pode-se também garantir que o problema está bem posto em um espaço de potência fracionária que possui imersão contínua no espaço das funções contínuas, como abordado em Oliva e Pereira (2002).

Uma vez que os problemas parabólicos estão bem postos, outras questões que aparecem estão relacionadas com a existência de atratores globais para esses problemas, bem como o comportamento assintótico desses atratores. Para tanto, existe um amplo estudo em torno dessas questões, que pode ser encontrado em Arrieta et al. (2000); Carvalho (2012); Carvalho et al. (1997) e Oliva e Pereira (2002).

Agora, uma questão mais recente, é a análise do comportamento assintótico de E.D.P's com termos concentrados na fronteira. A técnica de concentração foi inicialmente estudada por José M. Arrieta, Aníbal Rodríguez-Bernal e Ángela Jímenez-Casas em Arrieta et al. (2008), onde os autores analisaram o limite das soluções de um problema elíptico, posto em um domínio $\Omega \subset \mathbb{R}^{n}, n \geq 2$, quando alguns termos de reação e potencial estão concentrados em uma vizinhança $\omega_{\epsilon} \subset \bar{\Omega}, \epsilon>0$ suficientemente pequeno, de uma partição $\Gamma$ da fronteira e esta vizinhança contrai-se à $\Gamma$, quando o parâmetro $\epsilon \rightarrow 0$. Foi provado que essas soluções convergem em certos espaços de Bessel e no espaço das funções contínuas para a solução de um problema elíptico, onde o termo de reação e o potencial concentrado são transformados em uma condição de fluxo e um potencial em $\Gamma$.

As equações parabólicas não lineares com termos concentrados na fronteira foram estudadas em Jiménes-Casas e Rodríguez-Bernal (2009) e Jiménes-Casas e Rodríguez-Bernal (2011). Nesses artigos, os autores provaram que o problema limite de um problema parabólico não linear com termos concentrados numa vizinhança da fronteira e com condições de fronteira de Neumann homogêneas é dado por um problema parabólico não linear com condições de Robin (ou Neumann) não lineares, usando os resultados de Rodríguez-Bernal (2011), onde o caso linear foi estudado. Provaram também a existência e semicontinuidade superior da família de atratores e concluíram a semicontinuidade superior da família de equilíbrios. Em Jiménes-Casas e Rodríguez-Bernal (2011) e Rodríguez-Bernal (2011), é estudado o problema num espaço de Bessel $H_{p}^{s}(\Omega)$, com $s \in \mathbb{R}$ e $1<p<\infty$, já em Jiménes-Casas e Rodríguez-Bernal (2009), os autores estão interessados no espaço $H^{1}(\Omega)$. Cabe ressaltar que em Arrieta et al. (2008), Jiménes-Casas e Rodríguez-Bernal (2009, 2011) e Rodríguez-Bernal (2011) o domínio $\Omega$ é um conjunto aberto, limitado e suave em $\mathbb{R}^{n}, n \geq 2$, com uma fronteira $C^{2}$ e $\omega_{\epsilon} \subset \bar{\Omega}$. Além disso, em Jiménes-Casas e Rodríguez-Bernal $(2009,2011)$ foram assumidas condições de crescimento crítico nas não linearidades para garantir que os problemas estão bem postos.

A mesma técnica de Arrieta et al. (2008) foi utilizada em Aragao e Oliva (2011, 2012), onde os resultados obtidos em Jiménes-Casas e Rodríguez-Bernal (2009, 2011) foram estendidos para problemas de reação-difusão com retardo e, além disso, a continuidade da família de equilíbrios foi provada. Uma aplicação interessante desses problemas são os modelos de crescimento populacional como, por exemplo, o modelo dado pela equação logística.

Em Aragao et al. (2012) alguns resultados de Arrieta et al. (2008) foram adaptados para problemas elípticos não lineares, quando alguns termos estão concentrados em uma vizinhança $\omega_{\epsilon}$ da fronteira e esta vizinhança apresenta um comportamento altamente oscilatório, e ainda, a fronteira do domínio é somente Lipschitz-contínua, mais especificamente, o domínio é um quadrado aberto em $\mathbb{R}^{2}$. Depois, em Aragao et al. (2014) foi provado a continuidade dos atratores, no caso em que o domínio $\Omega \subset \mathbb{R}^{2}$ é um conjunto aberto, limitado com fronteira $C^{2}$, e $\omega_{\epsilon}$ apresenta um comportamento altamente oscilatório.

Em todos esses trabalhos citados com a técnica de concentração, o domínio $\Omega$ não está variando. Depois, em Aragao e Bruschi (2015), Aragao e Bruschi (2016) e Nogueira (2017), foram estudadas equações elípticas com termos concentrados na fronteira do domínio e este domínio por sua vez está 
variando.

Recentemente, em Aragao e Bezerra (2017) foi estudado o comportamento assintótico dos atratores pullback de uma equação da onda amortecida, não autônoma e não linear, com termos concentrados. Foi provado um resultado de regularidade dos atratores pullback e sua semicontinuidade superior, neste caso $\Omega \subset \mathbb{R}^{3}$ e $\omega_{\epsilon} \subset \bar{\Omega}$.

Apesar das diversas abordagens, os autores deixam claro a motivação do estudo desse tipo de equação: as aplicações, de forma que estudar Equações Diferenciais é de suma importância para as Ciências Naturais. Afinal, entender o comportamento ou encontrar a solução de um problema desse tipo é a mesma coisa que prever, sob determinadas situações, o comportamento de um fenômeno natural. Nesse caso, o problema limite do nosso problema concentrado é uma equação famosa conhecida como equação de reação-difusão, quando estamos trabalhando no âmbito da Química ou da dinâmica populacional, ou também, como a equação do calor, quando estamos trabalhando com a equação no âmbito da Física e termodinâmica. Além disso, os resultados de convergência envolvendo problemas concentrados, podem ser vistos como uma ferramenta de transferir informações do domínio $\Omega$ para a fronteira $\partial \Omega$.

Motivados pelos trabalhos citados, estamos interessados em estudar o comportamento assintótico de uma E.D.P parabólica não linear com condições de Neumann homogêneas e com termos concentrados em uma vizinhança $\omega_{\epsilon} \subset \bar{\Omega}$ da fronteira do domínio fixo $\Omega \subset \mathbb{R}^{n}, n \geq 2$, em $H^{1}(\Omega)$ e sob hipóteses de crescimento crítico das não linearidades. Dessa forma, nosso trabalho estará mais focado em Jiménes-Casas e Rodríguez-Bernal (2009, 2011).

\subsection{Objetivos}

A seguir, apresentaremos o problema que estamos interessados em estudar e comentaremos os principais resultados a serem comprovados.

Considere $\Omega \subset \mathbb{R}^{n}, n \geq 2$, um aberto limitado com fronteira $\partial \Omega$ de classe $C^{2}$. Definimos a faixa de largura $\epsilon$ e base $\partial \Omega$, como

$$
\omega_{\epsilon}=\{x-\sigma \vec{n}(x): x \in \partial \Omega \quad \text { e } \quad \sigma \in[0, \epsilon)\},
$$

para $\epsilon$ suficientemente pequeno, digamos $0<\epsilon \leq \epsilon_{0}$, onde $\vec{n}(x)$ é o vetor normal unitário exterior em $x \in \partial \Omega$. O conjunto $\omega_{\epsilon}$ tem medida de Lebesgue $\left|\omega_{\epsilon}\right|=O(\epsilon)$ com $\left|\omega_{\epsilon}\right| \leq k \epsilon|\partial \Omega|$, para algum $k>0$ independente de $\epsilon$. Além disso, para $\epsilon$ pequeno, $\omega_{\epsilon}$ é uma vizinhança da fronteira $\partial \Omega$ no $\bar{\Omega}$, que contrai-se à $\partial \Omega$ quando o parâmetro $\epsilon \rightarrow 0$. Ver Figura 1.1 .

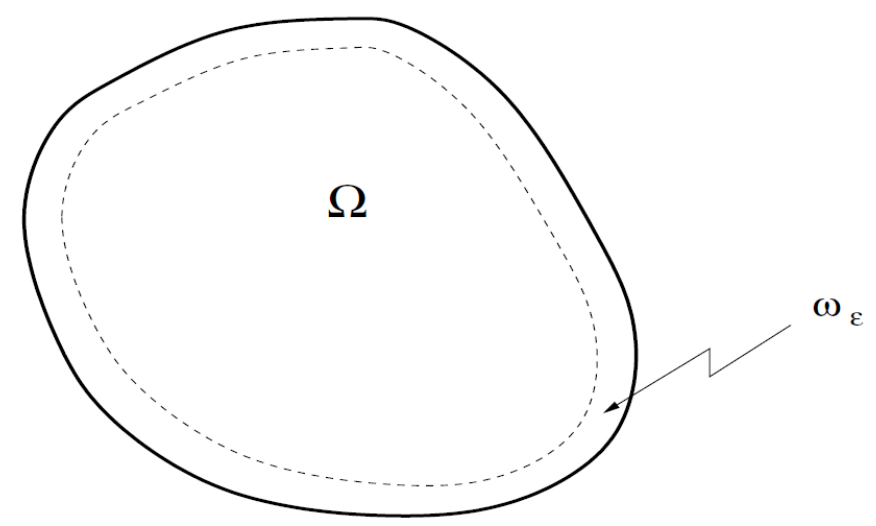

Figura 1.1: $O$ conjunto $\omega_{\epsilon}$, imagem retirada de Arrieta et al. (2008).

Estudaremos o comportamento, para $\epsilon$ pequeno, das soluções do problema parabólico não linear com condições de fronteira de Neumann homogêneas, dado por 


$$
\begin{cases}\frac{\partial u^{\epsilon}}{\partial t}(t, x)+L^{\epsilon} u^{\epsilon}(t, x)=f\left(x, u^{\epsilon}(t, x)\right)+\frac{1}{\epsilon} \chi_{\omega_{\epsilon}}(x) g\left(x, u^{\epsilon}(t, x)\right), & (t, x) \in(0, \infty) \times \Omega \\ \frac{\partial u^{\epsilon}}{\partial \vec{n}}(t, x)=0, & (t, x) \in(0, \infty) \times \partial \Omega \\ u^{\epsilon}(0, x)=u_{0}(x), & x \in \Omega\end{cases}
$$

onde $\chi_{\omega_{\epsilon}}$ é a função característica do conjunto $\omega_{\epsilon} \mathrm{e}$

$$
L^{\epsilon} u=-\Delta u+\mu u+\frac{1}{\epsilon} \chi_{\omega_{\epsilon}}(x) V_{\epsilon}(x) u
$$

com $u \in \mathbb{R}, \mu>0$ e $0<\epsilon \leq \epsilon_{0}$.

Nos refiremos aos termos $\frac{1}{\epsilon} \chi_{\omega_{\epsilon}}(x) g\left(x, u^{\epsilon}(t, x)\right)$ e $\frac{1}{\epsilon} \chi_{\omega_{\epsilon}}(x) V_{\epsilon}(x) u^{\epsilon}(t, x)$ como a reação e o potencial concentrados na faixa $\omega_{\epsilon}$.

Assumiremos a seguinte hipótese para o potencial:

(P) - Família de potenciais concentrados e convergentes.

Vamos assumir que existe $K_{1}>0$ independente de $\epsilon$ tal que

$$
\frac{1}{\epsilon} \int_{\omega_{\epsilon}}\left|V_{\epsilon}\right|^{\rho} \mathrm{d} x \leq K_{1}
$$

para algum $\rho>n-1$ e que existe uma função $V_{0} \in L^{\rho}(\partial \Omega)$ tal que

$$
\lim _{\epsilon \rightarrow 0} \frac{1}{\epsilon} \int_{\omega_{\epsilon}} V_{\epsilon} \phi \mathrm{d} x=\int_{\partial \Omega} V_{0} \phi \mathrm{d} S, \quad \forall \phi \in C^{\infty}(\bar{\Omega}) .
$$

Assumiremos também as seguintes hipóteses para as não linearidades:

(H1) - Condição de crescimento crítico.

Suponha que $f, g: \bar{\Omega} \times \mathbb{R} \rightarrow \mathbb{R}$ são funções uniformemente contínuas, $f(x, \cdot), g(x, \cdot): \mathbb{R} \rightarrow \mathbb{R}$ são localmente Lipschitzianas uniformemente em $x \in \Omega$ e $x \in \bar{\Omega}$, respectivamente, e satisfazem as seguintes condições de crescimento:

i. Se $n>2$, existe $c>0$ tal que

$$
|j(x, u)-j(x, v)| \leq c|u-v|\left(|u|^{\sigma_{j}}+|v|^{\sigma_{j}}+1\right),
$$

onde $j=f$ ou $j=g$ com expoentes $\sigma_{f}$ e $\sigma_{g}$, respectivamente, tais que

$$
\sigma_{f} \leq \frac{4}{n-2} \quad \text { e } \quad \sigma_{g} \leq \frac{2}{n-2} .
$$

ii. Se $n=2$, para qualquer $\eta>0$ existe $c_{\eta}>0$ tal que

$$
|j(x, u)-j(x, v)| \leq c_{\eta}|u-v|\left(e^{\eta|u|^{2}}+e^{\eta|v|^{2}}\right),
$$

onde $j=f$ ou $j=g$.

(H2) - Condição de sinal.

Suponha que existem funções $C \in L^{p}(\Omega), 0 \leq D \in L^{p}(\Omega)$, onde $p>\frac{n}{2}, E \in L^{q}(\Omega), 0 \leq F \in$ $L^{q}(\Omega)$, onde $q>n-1$, tais que

$$
\begin{array}{llll}
s f(x, s) \leq C(x) s^{2}+D(x)|s|, & x \in \Omega & \text { e } & s \in \mathbb{R}, \\
s g(x, s) \leq E(x) s^{2}+F(x)|s|, & x \in \bar{\Omega} & \text { e } & s \in \mathbb{R} .
\end{array}
$$


Além disso, para $\epsilon \in\left(0, \epsilon_{0}\right]$, existem constantes $K_{2}, K_{3}>0$ independentes de $\epsilon$ tais que

$$
\begin{gathered}
\frac{1}{\epsilon} \int_{\omega_{\epsilon}}|E|^{r_{1}} \mathrm{~d} x \leq K_{2}, \quad \text { onde } \quad r_{1}>n-1 ; \\
\frac{1}{\epsilon} \int_{\omega_{\epsilon}}|F|^{r_{2}} \mathrm{~d} x \leq K_{3}, \quad \text { onde } \quad r_{2}>\max \left\{1, \frac{2(n-1)}{n}\right\} .
\end{gathered}
$$

\section{(H3) - Condição de dissipação.}

Suponha que o primeiro autovalor, $\lambda_{1}$, do problema a seguir é positivo,

$$
\begin{cases}-\Delta \phi(x)-C(x) \phi(x)+\mu \phi(x)=\lambda_{1} \phi(x), & x \in \Omega \\ \frac{\partial \phi}{\partial \vec{n}}(x)+V_{0}(x) \phi(x)=E(x) \phi(x), & x \in \partial \Omega\end{cases}
$$

onde $C$ e $E$ são como em (H2).

Usando essas hipóteses, provaremos que o problema limite do problema concentrado (1.1) é dado pelo seguinte problema parabólico com condições de fronteira de Neumann não lineares

$$
\begin{cases}\frac{\partial u^{0}}{\partial t}(t, x)+L^{0} u^{0}(t, x)=f\left(x, u^{0}(t, x)\right), & (t, x) \in(0, \infty) \times \Omega \\ \frac{\partial u^{0}}{\partial \vec{n}}(t, x)+V_{0}(x) u^{0}(t, x)=g\left(x, u^{0}(t, x)\right), & (t, x) \in(0, \infty) \times \partial \Omega \\ u^{0}(0, x)=u_{0}(x), & x \in \Omega\end{cases}
$$

onde

$$
L^{0} u=-\Delta u+\mu u
$$

$\operatorname{com} u \in \mathbb{R}$ e $\mu>0$.

Usando a hipótese (H1) mostraremos a existência local e unicidade de soluções $u^{\epsilon}$ de (1.1), $0<$ $\epsilon \leq \epsilon_{0}$, e $u^{0}$ de $(1.4)$ em $H^{1}(\Omega)$ com a condição inicial $u_{0} \in H^{1}(\Omega)$, bem como a dependência contínua das soluções em relação aos dados iniciais. Depois, assumindo também a hipótese (H2) mostraremos que as soluções são globalmente definidas. Assumindo também a hipótese (H3) teremos uma família de atratores $\mathcal{A}_{\epsilon}$ em $H^{1}(\Omega), 0 \leq \epsilon \leq \epsilon_{0}$, para (1.1) e (1.4), e provaremos a semicontinuidade superior desta família em $\epsilon=0$. Por fim, concluiremos que a família de equilíbrios $\mathcal{E}_{\epsilon}$ em $H^{1}(\Omega), 0 \leq \epsilon \leq \epsilon_{0}$, dos problemas (1.1) e (1.4) é também semicontínua superiormente em $\epsilon=0$.

\subsection{Organização do trabalho}

O trabalho está organizado da seguinte forma:

- No Capítulo 2:

a) Nas Seções 2.1, 2.2 e 2.3, estaremos interessados em discutir os fundamentos da Análise Funcional (ver Brezis (2010); Yosida (1980)) e teoria de semigrupos (ver Brezis (2010); Carvalho (2012, 2001); Henry (1981); Yosida (1980)). Além disso, na Seção 2.4 vamos tentar introduzir alguns elementos e resultados da teoria de interpolação aplicados a teoria de semigrupo e operadores lineares (ver Amann (1995); Triebel (1978));

b) Na Seção 2.5, vamos introduzir resultados de existência local e unicidade de soluções de equações parabólicas, bem como resultados que garantem que essa solução é globalmente definida (ver Carvalho et al. (1997); Czaja (2002); Henry (1981); Yagi (2010));

c) Na Seção 2.6, vamos discutir a teoria de sistemas dinâmicos, em específico, a teoria de atratores (ver Carvalho (2012); Hale (1988); Henry (1981); Oliva e Pereira (2002); Temam (1988)); 
d) Nas Seções 2.7 e 2.8 discutiremos a noção de termos concentrados e seus principais resultados (ver Arrieta et al. (2008)) e aplicações à problemas elípticos.

- No Capítulo 3, estaremos interessados em aplicar a teoria do Capítulo 2 nos problemas (1.1) e (1.4):

a) Primeiro, na Seção 3.1, obtemos a formulação abstrata dos problemas (1.1) e (1.4);

b) Nas Seções 3.2 e 3.3, mostraremos que os problemas (1.1) e (1.4) estão bem postos assumindo a condição de crescimento (H1) e, utilizando (H2), mostraremos que as soluções de (1.1) e (1.4) estão globalmente definidas e, também assumindo (H3), as soluções são uniformemente limitadas em $H^{1}(\Omega)$ e $L^{\infty}(\Omega)$ para todo $t \geq 0$.

- No Capítulo 4, estaremos interessados em estudar o comportamento assintótico de (1.1) e (1.4):

a) Primeiro, na Seção 4.1, mostraremos a convergência das não linearidades e do semigrupo não linear associado às soluções de (1.1) e (1.4);

b) Já na Seção 4.2, estudaremos a família de atratores de (1.1) e (1.4) e a semicontinuidade superior dessa família;

c) Já na Seção 4.3, vamos estudar os equilíbrios de (1.1) e (1.4). Apresentaremos a formulação abstrata dos problemas elípticos associados às soluções de equilíbrios de (1.1) e (1.4), bem como mostraremos que a família de equilíbrios de (1.1) e (1.4) é semicontínua superiormente;

d) Na última Seção 4.4, vamos fazer um resumo dos resultados obtidos e as perspectivas para futuros trabalhos.

- No Apêndice A, vamos discutir outra abordagem para provar a condição de Lipschitz. 


\section{Capítulo 2}

\section{Notações e preliminares}

Ao estudar um problema de Equações Diferenciais procura-se estabelecer algumas conclusões quanto a existência, unicidade, regularidade e propriedades dinâmicas das soluções. Assim, surge, naturalmente, a necessidade de introduzir alguns espaços no qual faz sentido buscar soluções.

Classicamente, tenta-se exibir soluções com classes de diferenciabilidade pelo menos igual a ordem de diferenciabilidade do problema e que satisfazem todas as hipóteses exigidas (de contorno e/ou de valor inicial), essa é a noção de uma solução clássica.

Como exemplo, temos o problema de Laplace com condição de contorno de Dirichlet:

$$
\begin{cases}\Delta u(x)=0, & x \in B_{1}(0) \\ u(x)=g(x), & x \in \partial B_{1}(0)\end{cases}
$$

onde $g: \mathbb{R}^{n} \rightarrow \mathbb{R}$ é uma função contínua na bola unitária fechada $\overline{B_{1}(0)} \subset \mathbb{R}^{n}$.

Nesse caso, é possível exibir uma solução clássica para esse problema usando o núcleo de Poisson para a bola unitária e, com alguma formulação do príncipio do máximo, é possível obter a unicidade numa determinada classe de soluções.

Contudo, nem sempre é possível encontrar uma solução no sentido clássico em problemas elípticos, parabólicos ou hiperbólicos, então utiliza-se a Análise Funcional e a teoria de semigrupos para olhar o problema como uma equação de evolução em um espaço de Banach (isto é, espaço normado completo). Para tanto, o problema é reformulado de uma forma abstrata e a escolha de alguns espaços funcionais adequados auxilia a obter soluções num sentido mais fraco e resolver o problema.

Uma vez que o problema abstrato está bem posto, isto é, as soluções existem, são únicas e dependem continuamente do dado inicial, busca-se estudar as propriedades qualitativas do problema como, por exemplo, a existência de equilíbrios e atratores, bem como o comportamento assintótico dos atratores.

Este trabalho tem como objetivo investigar as questões discutidas acima. Dessa forma, este capítulo será destinado a introduzir, de uma forma resumida, alguns resultados preliminares e notações que usaremos ao longo deste trabalho. Mais detalhes e demonstrações dos resultados que serão expostos aqui podem ser encontrados nas bibliografias citadas.

\subsection{Operadores lineares}

As definições e resultados a seguir são discutidas por Brezis (2010) e Yosida (1980).

Como vamos trabalhar com a teoria de operadores, veremos algumas definições e notações. Sejam $E$ um espaço de Banach com norma $\|\cdot\|_{E}, A: D(A) \subset E \rightarrow E$ um operador linear, isto é,

- $A(u+v)=A(u)+A(v), \quad \forall u, v \in D(A)$,

- $A(\lambda u)=\lambda A(u), \quad \forall u \in D(A) \quad$ e $\quad \forall \lambda \in \mathbb{K}$, 
onde $\mathbb{K}=\mathbb{R}$ ou $\mathbb{K}=\mathbb{C}$, e denota-se por $D(A)$, o domínio de $\mathrm{A}, G(A)$, o gráfico de $\mathrm{A}, R(A)$, a imagem de A e, $N(A)$, o núcleo de A. Dizemos que:

1. A é limitado (ou contínuo) se $\exists c>0$ tal que $\|A(u)\|_{E} \leq c\|u\|_{E}, \forall u \in D(A)$, caso contrário, $A$ é ilimitado;

2. $A$ é fechado se $G(A) \subset E \times E$ é um subconjunto fechado;

3. $A$ é densamente definido se $D(A)$ é denso em $E$;

4. A é compacto se $A(K)$ possui fecho, $\overline{A(K)}$, compacto em $E$, para todo subconjunto $K \subset D(A)$ limitado.

Em espaços de dimensão finita, a noção de autovalor e autovetor de uma transformação $A$ está associada aos valores $\lambda \in \mathbb{K}$ tais que $A(u)=\lambda u$, onde $u \neq 0$ e $u \in D(A)$. Para discutir a ideia de autovalores em dimensão infinita será introduzido o conjunto resolvente e o conjunto espectral.

Considere $A: D(A) \subset E \rightarrow E$, um operador linear, onde $E$ é um espaço de Banach, $I$ o operador identidade, $\lambda \in \mathbb{K}$ e o operador

$$
A_{\lambda}=A-\lambda I .
$$

Sob as condições acima, o conjunto de todos os valores $\lambda \in \mathbb{K}$ tais que $R\left(A_{\lambda}\right)$ é denso em $E$ e $A_{\lambda}$ possui uma inversa $(A-\lambda I)^{-1}$ contínua, é definido como o conjunto resolvente de $A$ e denotado por $\rho(A)$.

O espectro de A é o complemento do resolvente, isto é,

$$
\sigma(A)=\mathbb{C} \backslash \rho(A) .
$$

O conjunto espectral $\sigma(A)$ pode ser decomposto em subconjuntos disjuntos

1. $P_{\sigma(A)}$, o espectro pontual. É o conjunto de $\lambda \in \mathbb{K}$ tais que $A_{\lambda}$ não é inversível;

2. $C_{\sigma(A)}$, o espectro contínuo. É o conjunto de $\lambda \in \mathbb{K}$ tais que $A_{\lambda}$ possui inversa descontínua e $R\left(T_{\lambda}\right)$ é denso em $E$;

3. $R_{\sigma(A)}$, o espectro residual. É o conjunto de $\lambda \in \mathbb{K}$ tais que $A_{\lambda}$ possui inversa e $R\left(A_{\lambda}\right)$ não é denso em $E$.

Neste trabalho, vamos denotar por $\mathcal{L}(E, F)$ o espaço dos operadores lineares e contínuos de $E$ em $F$, onde $E$ e $F$ são espaços de Banach. O espaço $\mathcal{L}(E, F)$ é um espaço de Banach com a norma usual

$$
\|A\|_{\mathcal{L}(E, F)}=\sup _{u \in E}\|A(u)\|_{F} .
$$

Se $E=F$ escreveremos $\mathcal{L}(E)$ para denotar $\mathcal{L}(E, E)$. Seja $E^{\prime}$ o dual topológico de $E$, isto é, $E^{\prime}=\mathcal{L}(E, \mathbb{K})$, onde $\mathbb{K}=\mathbb{R}$ ou $\mathbb{K}=\mathbb{C}$, com a topologia dada pela norma acima. Denotaremos o valor de $\phi \in E^{\prime}$ em $u \in E$ por $\langle\phi, u\rangle$.

\subsection{Espaços funcionais}

As definições e resultados a seguir são discutidas por Haroske e Triebel (2008), Adams (1975), Brezis (2010), Triebel (1978) e Yagi (2010).

Considere $\Omega \subset \mathbb{R}^{n}$ um aberto ou $\Omega=\mathbb{R}^{n}$ e quando necessário faremos a distinção. Alguns resultados enunciados aqui podem ser obtidos para domínios $\Omega \subset \mathbb{R}^{n}$ com fronteira menos regulares, como por exemplo, Lipschitz. Como vamos trabalhar com fronteira suave, não abordaremos essa questão aqui. Para mais detalhes ver, por exemplo, Yagi (2010). 


\subsubsection{O espaço das funções contínuas, $C^{0}(\Omega)$}

Esse espaço é formado por todas as funções $u: \Omega \rightarrow \mathbb{R}$ que são contínuas. Escolhendo a norma do supremo, isto é,

$$
\|u\|_{C^{0}(\Omega)}=\sup _{x \in \Omega}|u(x)|,
$$

segue que $C^{0}(\Omega)$ é um espaço de Banach.

\subsubsection{O espaço das funções Hölder contínuas, $C^{0, l}(\Omega)$}

O espaço das funções Hölder contínuas $C^{0, l}(\Omega), 0<l \leq 1$, é constituído de todas as funções que satisfazem

$$
|u(x)-u(y)| \leq K|x-y|^{l}, \quad \forall x, y \in \Omega,
$$

para algum $K>0$. Se $l=1$ dizemos que a função é Lipschitziana. Escolhendo a norma

$$
\|u\|_{C^{0, l}(\Omega)}=\sup _{\substack{x, y \in \Omega \\ x \neq y}}|u(x)|+\sup _{\substack{x, y \in \Omega \\ x \neq y}} \frac{|u(x)-u(y)|}{|x-y|^{l}}
$$

segue que $C^{0, l}(\Omega)$ é um espaço de Banach.

\subsubsection{O espaço das funções de classe $m$-diferenciáveis, $C^{m}(\Omega)$}

Seja $m \in \mathbb{N}$ e considere o multi-índice $\alpha=\left(\alpha_{1}, \ldots, \alpha_{n}\right)$, isto é, uma $n$-upla de inteiros não negativos. Definimos:

- $|\alpha|=\alpha_{1}+\ldots+\alpha_{n} ;$

- $\alpha !=\left(\alpha_{1} !\right) \cdot\left(\alpha_{2} !\right) \ldots .\left(\alpha_{n} !\right)$;

- $x^{\alpha}=x_{1}^{\alpha_{1}} x_{2}^{\alpha_{2}} \ldots x_{n}^{\alpha_{n}}$, onde $x=\left(x_{1}, \ldots, x_{n}\right) \in \mathbb{R}^{n}$;

- $D^{\alpha}=\partial_{1}^{\alpha_{1}} \ldots \partial_{n}^{\alpha_{n}}$, onde $\partial_{i}^{\alpha_{i}}=\frac{\partial^{\alpha_{i}}}{\partial x_{i}^{\alpha_{i}}}, \quad \forall i=1, . ., n$.

O espaço das funções de classe $m$-diferenciáveis consiste de todas as funções $u: \Omega \rightarrow \mathbb{R}$ que são $m$-diferenciáveis e as $m$-derivadas são contínuas. Tomando a norma

$$
\|u\|_{C^{m}(\Omega)}=\sum_{|\alpha|=0}^{m} \sup _{x \in \Omega}\left|D^{\alpha} u(x)\right|,
$$

segue que $C^{m}(\Omega)$ é um espaço de Banach.

\subsubsection{O espaço das funções infinitamente diferenciáveis, $C^{\infty}(\Omega)$}

Quando $u$ é infinitamente diferenciável dizemos que $u$ é uma função suave. Vamos definir formalmente como

$$
C^{\infty}(\Omega)=\bigcap_{m=0}^{\infty} C^{m}(\Omega) .
$$

Sejam $u$ uma função suave e $\operatorname{supp}(u)$ o suporte de $u$, isto é, $\operatorname{supp}(u)=\overline{\{x \in \Omega: u(x) \neq 0\}}$. Vamos denotar por $\mathcal{D}(\Omega)$, o espaço das funções suaves com suporte compacto em $\Omega$, isto é, $\operatorname{supp}(u)$ é um subconjunto compacto de $\Omega$. Vamos chamar $\mathcal{D}(\Omega)$ por espaço das funções teste. 


\subsubsection{O espaço das funções $p$-Lebesgue integráveis, $L^{p}(\Omega)$}

Além desses espaços relacionados a diferenciabilidade, também define-se, sobre as classes de equivalências das funções Lebesgue mensuráveis, o espaço de Lebesgue das funções $p$-integráveis. Considere o corpo $\mathbb{K}=\mathbb{R}$ ou $\mathbb{K}=\mathbb{C}$.

Caso $1 \leq p<\infty$ :

$$
L^{p}(\Omega)=\left\{u: \Omega \rightarrow \mathbb{K} \quad \text { Lebesgue mensurável : } \int_{\Omega}|u(x)|^{p} \mathrm{~d} x<\infty\right\} .
$$

Escolhendo a norma

$$
\|u\|_{L^{p}(\Omega)}=\left(\int_{\Omega}|u(x)|^{p} \mathrm{~d} x\right)^{\frac{1}{p}},
$$

segue que $L^{p}(\Omega)$ é um espaço de Banach.

Caso $p=\infty$, obtemos o espaço das funções Lebesgue mensuráveis essencialmente limitadas,

$$
L^{\infty}(\Omega)=\left\{u: \Omega \rightarrow \mathbb{K} \quad \text { Lebesgue mensurável : } \sup _{x \in \Omega}|u(x)|<\infty\right\} .
$$

Escolhendo a norma do supremo

$$
\|u\|_{L^{\infty}(\Omega)}=\sup _{x \in \Omega}|u(x)|,
$$

segue que $L^{\infty}(\Omega)$ também é um espaço de Banach.

Em particular, o espaço $L^{2}(\Omega)$ é um espaço de Hilbert, isto é, é possível munir o espaço com uma aplicação $\langle,\rangle_{L^{2}(\Omega)}: L^{2}(\Omega) \times L^{2}(\Omega) \rightarrow \mathbb{K}$ chamada de produto interno que é dada por

$$
<u, v>_{L^{2}(\Omega)}=\int_{\Omega} u(x) \overline{v(x)} \mathrm{d} x, \quad u, v \in L^{2}(\Omega) .
$$

Também vamos definir $L_{l o c}^{p}(\Omega)$ como o espaço das funções Lebesgue mensuráveis que são localmente $p$-integráveis em $\Omega$, isto é, em subconjuntos $K \subset \Omega$ obtemos que

$$
\int_{K}|u(x)|^{p} \mathrm{~d} x<\infty
$$

Teorema 2.2.1 (Desigualdade de Hölder) Se $u \in L^{p}(\Omega)$ e $v \in L^{q}(\Omega)$, onde $1<p, q,<\infty e$ $\frac{1}{p}+\frac{1}{q}=1$, ou $p=1$ e $q=\infty$, então $u v \in L^{1}(\Omega) e$

$$
\|u v\|_{L^{1}(\Omega)} \leq\|u\|_{L^{p}(\Omega)}\|v\|_{L^{q}(\Omega)} .
$$

Se $p=q=2$ então a desigualdade acima é chamada de desigualdade de Cauchy-Schwartz.

Teorema 2.2.2 (Desigualdade de Minkowski) Se $u, v \in L^{p}(\Omega)$, onde $1 \leq p, q, \leq \infty$, então $u+v \in L^{p}(\Omega) e$

$$
\|u+v\|_{L^{p}(\Omega)} \leq\|u\|_{L^{p}(\Omega)}+\|v\|_{L^{p}(\Omega)} .
$$

\subsubsection{O espaço das distribuições, $\mathcal{D}^{\prime}(\Omega)$}

Considere o corpo $\mathbb{K}=\mathbb{R}$ ou $\mathbb{K}=\mathbb{C}, \mathcal{D}(\Omega)$ e seu dual $\mathcal{D}^{\prime}(\Omega)$, o espaço de todos os funcionais lineares e contínuos

$$
T: \mathcal{D}(\Omega) \rightarrow \mathbb{K},
$$

isto é, $T \in \mathcal{D}^{\prime}(\Omega)$, é chamado de espaço das distribuições.

A distribuição $T$ é dita regular se existe $f \in L_{l o c}^{1}(\Omega)$ tal que $T=T_{f}$, onde

$$
T_{f} \phi=\int_{\Omega} f(x) \phi(x) \mathrm{d} x, \quad \phi \in \mathcal{D}(\Omega),
$$


caso contrário, $T$ é dita singular.

Por fim, dado $\alpha$, um multi-índice, define-se a derivada de $T \in \mathcal{D}^{\prime}(\Omega)$ no sentido das distribuições por

$$
\left(D^{\alpha} T\right) \phi=(-1)^{|\alpha|} T\left(D^{\alpha} \phi\right), \quad \phi \in \mathcal{D}(\Omega) .
$$

\subsubsection{O espaço de Schwartz, $\mathcal{S}\left(\mathbb{R}^{n}\right)$}

O espaço de Schwartz, $\mathcal{S}\left(\mathbb{R}^{n}\right)$, contém todas as funções $u \in C^{\infty}\left(\mathbb{R}^{n}\right)$ que decaem mais rapidamente no infinito do que qualquer polinômio. O espaço $\mathcal{S}\left(\mathbb{R}^{n}\right)$ é de Banach com a norma

$$
\|u\|_{k, l}=\sup _{x \in \mathbb{R}^{n}}\left\{\left(1+|x|^{2}\right)^{\frac{k}{2}} \sum_{|\alpha| \leq l}\left|D^{\alpha} u(x)\right|\right\}, \quad \forall k, l \in \mathbb{N} .
$$

As distribuições assumem um papel importante no espaço de Schwartz, pois seu dual topológico, denotado por $\mathcal{S}^{\prime}\left(\mathbb{R}^{n}\right)$, é chamado de espaço das distribuições temperadas.

O espaço de Schwartz possui algumas propriedades importantes relacionadas a transformada de Fourier, que veremos a seguir.

\subsubsection{Transformada de Fourier}

Considere $u \in \mathcal{S}\left(\mathbb{R}^{n}\right)$ e $\xi \in \mathbb{R}^{n}$, a transformada de Fourier e a transformada inversa de $u$ são definidas, respectivamente, por

$$
\begin{aligned}
& (\mathcal{F} u)(\xi)=\hat{u}(\xi)=(2 \pi)^{-\frac{n}{2}} \int_{\mathbb{R}^{n}} e^{-i x \cdot \xi} u(x) \mathrm{d} x, \\
& \left(\mathcal{F}^{-1} u\right)(\xi)=\check{u}(\xi)=(2 \pi)^{-\frac{n}{2}} \int_{\mathbb{R}^{n}} e^{i x \cdot \xi} u(x) \mathrm{d} x,
\end{aligned}
$$

onde $x \cdot \xi=x_{1} \xi_{1}+\ldots+x_{n} \xi_{n}$, isto é, o produto interno usual do espaço euclidiano.

Se $T \in \mathcal{S}^{\prime}\left(\mathbb{R}^{n}\right)$, podemos definir a transformada de Fourier como

$$
\mathcal{F} T(u)=T(\mathcal{F}(u)), \quad u \in \mathcal{S}\left(\mathbb{R}^{n}\right) .
$$

De forma análoga para a transformada inversa.

Teorema 2.2.3 (Haroske e Triebel (2008), Teorema 2.42.) Seja $u \in \mathcal{S}\left(\mathbb{R}^{n}\right)$, entâo

$$
u(x)=\left(\mathcal{F F}^{-1} u\right)(x)=\left(\mathcal{F}^{-1} \mathcal{F} u\right)(x), \quad \forall x \in \mathbb{R}^{n} .
$$

Além disso, a transformada de Fourier é um automorfismo de $\mathcal{S}\left(\mathbb{R}^{n}\right)$.

Teorema 2.2.4 (Haroske e Triebel (2008), Teorema 2.60) Seja $T \in \mathcal{S}^{\prime}\left(\mathbb{R}^{n}\right)$, então

$$
T=\mathcal{F F}^{-1} T=\mathcal{F}^{-1} \mathcal{F} T \text {. }
$$

Além disso, a transformada de Fourier é um automorfismo de $\mathcal{S}^{\prime}\left(\mathbb{R}^{n}\right)$.

Dependendo da dificuldade do problema é necessário introduzir outros espaços com menos regularidade, e esses serão utilizados para nosso problema: os espaços de Sobolev e os espaços Potenciais de Bessel.

\subsubsection{Espaços de Sobolev em $\mathbb{R}^{n}$ e $\Omega \subset \mathbb{R}^{n}$ aberto}

Nas definições dadas a seguir $\Omega \subset \mathbb{R}^{n}$ é um aberto ou $\Omega=\mathbb{R}^{n}$, quando necessário faremos a distinção. 
Definição 2.2.5 (Espaço de Sobolev Clássico) Seja $k \in \mathbb{N}, \alpha$ um multi-índice e $1 \leq p<\infty$, definimos

$$
W_{p}^{k}(\Omega)=\left\{u \in L^{p}(\Omega): D^{\alpha} u \in L^{p}(\Omega), \quad \forall|\alpha| \leq k\right\}
$$

como o espaço clássico de Sobolev.

Teorema 2.2.6 (Adams (1975), Teorema 3.2) Sob a definiçẫo acima, o espaço $W_{p}^{k}(\Omega)$ com a norma

$$
\|u\|_{W_{p}^{k}(\Omega)}=\left(\sum_{|\alpha| \leq k}\left\|D^{\alpha} u\right\|_{L^{p}(\Omega)}\right)^{\frac{1}{p}}
$$

é um espaço de Banach.

Se $p=2, W_{2}^{k}(\Omega)$ é um espaço de Hilbert, munido do produto interno

$$
\langle u, v\rangle_{W_{2}^{k}(\Omega)}=\sum_{|\alpha| \leq k}\left\langle D^{\alpha} u, D^{\alpha} v\right\rangle_{L_{2}(\Omega)}, \quad u, v \in W_{2}^{k}(\Omega)
$$

Vamos estender o espaço de Sobolev clássico para $s \in \mathbb{R}^{+}$.

Definição 2.2.7 (Extensão do espaço de Sobolev Clássico) Seja $s=k+\sigma$, onde $k \in \mathbb{N}$, $\sigma \in(0,1)$ e $1 \leq p<\infty$. Então, define-se

$W_{p}^{s}(\Omega)=\left\{u \in \mathcal{D}^{\prime}(\Omega): u \in W_{p}^{k}(\Omega) \quad e \quad \iint_{\Omega \times \Omega} \frac{\left|D^{\alpha} u(x)-D^{\alpha} u(y)\right|^{p}}{|x-y|^{n+p \sigma}} d x d y<\infty \quad\right.$ para $\left.\quad|\alpha|=k\right\}$.

Definimos a norma em $W_{p}^{s}(\Omega)$ que o torna Banach, como:

$$
\|u\|_{W_{p}^{s}(\Omega)}=\left(\|u\|_{W_{p}^{k}(\Omega)}^{p}+\sum_{|\alpha|=k} \iint_{\Omega \times \Omega} \frac{\left|D^{\alpha} u(x)-D^{\alpha} u(y)\right|^{p}}{|x-y|^{n+p \sigma}} d x d y\right)^{\frac{1}{p}} .
$$

Definiremos para $s \in \mathbb{R}^{-}$, nesta situação, vamos dividir em casos, pois há diferença entre $\mathbb{R}^{n}$ e $\Omega \subset \mathbb{R}^{n}$ aberto. Para os resultados a seguir, considere $\frac{1}{p}+\frac{1}{q}=1$, com $1<p, q<\infty$, também vamos denotar o espaço dual $\left(L^{p}(\Omega)\right)^{\prime}=L^{q}(\Omega)$ (Ver Adams (1975), Lema 3.7).

Para $\mathbb{R}^{n}$ segue do espaço dual, isto é,

Definição 2.2.8 Para $s<0$ denotamos por $W_{p}^{s}\left(\mathbb{R}^{n}\right)$ o espaço dual de $W_{q}^{-s}\left(\mathbb{R}^{n}\right)$.

Já para o $W_{p}^{s}(\Omega), \Omega \subset \mathbb{R}^{n}$ aberto, primeiro definimos

Definição 2.2.9 Para $s>0$ denotamos por $\tilde{W}_{p}^{s}(\Omega)$ o fecho de $\mathcal{D}(\Omega)$ em $W_{p}^{s}(\Omega)$.

Por fim,

Definição 2.2.10 Para $s<0$ denotamos por $W_{p}^{s}(\Omega)$ o espaço dual de $\tilde{W}_{q}^{-s}(\Omega)$.

Uma caracterização mais detalhada dos espaços de Sobolev com potências negativas pode ser vistas em Yagi (2010) e Adams (1975). 


\subsubsection{Espaços Potencial de Bessel em $\mathbb{R}^{n}$ e $\Omega \subset \mathbb{R}^{n}$ aberto}

Para definir o espaço de Bessel, considere $w_{s}=\left(1+|x|^{2}\right)^{\frac{s}{2}}$, onde $x \in \mathbb{R}^{n}$ e $s \in \mathbb{R}$, e defina sobre o espaço das distribuições temperadas a aplicação:

$$
J^{s} u=\mathcal{F}^{-1}\left(w_{s} \mathcal{F} u\right), \quad u \in \mathcal{S}^{\prime}\left(\mathbb{R}^{n}\right),
$$

onde $J^{s}$ é chamado de potencial de Bessel de $u$ de ordem $s$.

Se $s>0$ e $1 \leq p \leq \infty$ ou, se $s \geq 0$ e $1<p<\infty$, o potencial de Bessel, $J^{s}$, transforma continuamente $L^{p}(\Omega)$ em $L^{p}(\Omega)$, e o mesmo acontece para $D^{\alpha} J^{s+|\alpha|}$, onde $\alpha$ é um multi-índice (ver Adams (1975), pág 220-221).

Definição 2.2.11 (Potencial de Bessel em $\mathbb{R}^{n}$ ) Sejam $s \in \mathbb{R} e 1<p<\infty$. Definimos

$$
H_{p}^{s}\left(\mathbb{R}^{n}\right)=\left\{u \in \mathcal{S}^{\prime}\left(\mathbb{R}^{n}\right): J^{s} u \in L^{p}\left(\mathbb{R}^{n}\right)\right\}
$$

como o espaço Potencial de Bessel.

Assim como todos os espaços citados anteriormente, o Potencial de Bessel é um espaço de Banach munido da norma

$$
\|u\|_{H_{p}^{s}\left(\mathbb{R}^{n}\right)}=\left\|J^{s} u\right\|_{L^{p}\left(\mathbb{R}^{n}\right)} .
$$

No caso $p=2$ usaremos a notação $H^{s}\left(\mathbb{R}^{n}\right)$ ao invés de $H_{2}^{s}\left(\mathbb{R}^{n}\right)$. Neste caso, os espaços são Hilbert.

O resultado a seguir relaciona os espaços de Bessel e Sobolev, os expoentes dos espaços de Bessel com seu dual e as respectivas imersões.

Teorema 2.2.12 (Adams (1975), Teorema 7.63) Valem os seguintes resultados:

1. Se $s \in \mathbb{R} e 1<p<\infty$, então $\mathcal{D}\left(\mathbb{R}^{n}\right)$ é denso em $H_{p}^{s}\left(\mathbb{R}^{n}\right)$;

2. Se $s \in \mathbb{R}, 1<p<\infty e \frac{1}{p}+\frac{1}{q}=1$, ent $\tilde{a} o\left(H_{p}^{s}\left(\mathbb{R}^{n}\right)\right)^{\prime}=H_{q}^{-s}\left(\mathbb{R}^{n}\right)$;

3. Se $t<s$ e $1<p<\infty$, então $H_{p}^{s}\left(\mathbb{R}^{n}\right) \hookrightarrow H_{p}^{t}\left(\mathbb{R}^{n}\right)$;

4. Se $t \leq s$ e também $1<p \leq q \leq \frac{n p}{n-(s-t) p}<\infty$ ou $p=1$ e $1 \leq q<\frac{n}{n-s+t}$, entâa $H_{p}^{s}\left(\mathbb{R}^{n}\right) \hookrightarrow H_{q}^{t}\left(\mathbb{R}^{n}\right) ;$

5. $H_{p}^{s}\left(\mathbb{R}^{n}\right)=W_{p}^{s}\left(\mathbb{R}^{n}\right)$ se, e somente se, $s \in \mathbb{R}$ e $p=2$ ou, $s \in \mathbb{Z}$ e $1<p<\infty$;

6. Se $0 \leq l \leq s-\frac{n}{p}<1$, então $H_{p}^{s}\left(\mathbb{R}^{n}\right) \hookrightarrow C^{0, l}\left(\mathbb{R}^{n}\right)$;

7. Dado $1<p<\infty$ e $\delta>0$, então para todo $s \in \mathbb{R}, H_{p}^{s+\delta}\left(\mathbb{R}^{n}\right) \hookrightarrow W_{p}^{s}\left(\mathbb{R}^{n}\right) \hookrightarrow H_{p}^{s-\delta}\left(\mathbb{R}^{n}\right)$.

Agora, iremos definir os espaços de Bessel para um $\Omega \subset \mathbb{R}^{n}$ arbitrário por restrição. Depois, definiremos por interpolação.

Considere o operador restrição em relação à $\Omega, r_{\Omega} \in \mathcal{L}\left(\mathcal{S}^{\prime}\left(\mathbb{R}^{n}\right), \mathcal{D}^{\prime}(\Omega)\right)$, dado por

$$
\begin{aligned}
r_{\Omega}: \mathcal{S}^{\prime}\left(\mathbb{R}^{n}\right) & \rightarrow \mathcal{D}^{\prime}(\Omega) \\
v & \mapsto r_{\Omega} v
\end{aligned}
$$

tal que

$$
\left\langle r_{\Omega} v, \phi\right\rangle=\langle v, \phi\rangle, \quad v \in \mathcal{S}^{\prime}\left(\mathbb{R}^{n}\right) \quad \text { e } \quad \phi \in D(\Omega) .
$$

Definição 2.2.13 (Potencial de Bessel em domínios) Sejam $\Omega \subset \mathbb{R}^{n}, 1<p<\infty$ es $\in \mathbb{R}$. Então, define-se o espaço Potencial de Bessel como

$$
H_{p}^{s}(\Omega)=r_{\Omega} H_{p}^{s}\left(\mathbb{R}^{n}\right)
$$


O Potencial de Bessel, $H_{p}^{s}(\Omega)$, munido da norma

$$
\|u\|_{H_{p}^{s}(\Omega)}=\inf _{\substack{v \\ u \\ u \\ u \\=H_{\Omega}^{s}\left(\mathbb{R}^{n}\right)}}\|v\|_{H_{p}^{s}\left(\mathbb{R}^{n}\right)}
$$

é um espaço de Banach para todo $s \in \mathbb{R}$. Caso $p=2, H_{2}^{s}(\Omega)$ também é um espaço de Hilbert e será denotado por $H^{s}(\Omega)$.

Uma forma equivalente de definir o espaço $H_{p}^{s}(\Omega)$ (ver Adams (1975)) é utilizando a teoria de interpolação (ver Triebel (1978), pág.56, ver também Yagi (2010)). Considere que $E_{0}$ e $E_{1}$ são espaços de Banach satisfazendo $E_{1} \hookrightarrow E_{0}$ densamente, com essas condições $\left(E_{0}, E_{1}\right)$ é chamado de par de interpolação. Também definimos a faixa no plano complexo

$$
S=\{z \in \mathbb{C}: 0<\operatorname{Re}(z)<1\} .
$$

Vamos denotar por $\mathcal{H}\left(E_{0}, E_{1}\right)$ o espaço das funções analíticas com as seguintes propriedades:

1. Se $F \in \mathcal{H}\left(E_{0}, E_{1}\right)$ então $F(z)$ é analítica para $z \in S$ com valores em $E_{0}$ e é contínua e limitada para $z=1+i y$ com valores em $E_{1}$.

2. Definindo a norma

$$
\|F\|_{\mathcal{H}\left(E_{0}, E_{1}\right)}=\max _{j=0,1}\left\{\sup _{y \in \mathbb{R}}\left\{\|F(j+i y)\|_{E_{j}}\right\}\right\}, \quad F \in \mathcal{H}\left(E_{0}, E_{1}\right)
$$

segue que $\mathcal{H}\left(E_{0}, E_{1}\right)$ é um espaço de Banach.

Dado $\left(E_{0}, E_{1}\right)$ um par de interpolação, para cada $\theta \in[0,1]$ definimos o espaço

$$
E_{\theta}=\left[E_{0}, E_{1}\right]_{\theta}=\left\{U \in E_{0}: \exists F \in \mathcal{H}\left(E_{0}, E_{1}\right) \text { tal que } \quad U=F(\theta)\right\} .
$$

A norma a seguir torna $E_{\theta}$ um espaço de Banach

$$
\|U\|_{E_{\theta}}=\inf _{\substack{F \in \mathcal{H}\left(E_{0}, E_{1}\right) \\ U=F(\theta)}}\|F\|_{\mathcal{H}\left(E_{0}, E_{1}\right)}, \quad U \in E .
$$

Segue algumas propriedades:

Teorema 2.2.14 (Propriedades de interpolação, Triebel (1978), pág 58-59) Sob as notações acima, são válidas:

1. $\left[E_{0}, E_{1}\right]_{0}=E_{0}$ e $\left[E_{0}, E_{1}\right]_{1}=E_{1}$ isometricamente;

2. Se $0<\theta<1, E_{1} \hookrightarrow E_{\theta} \hookrightarrow E_{0}$ densamente;

3. Se $0<\theta<1$. $\|U\|_{E_{\theta}} \leq\|U\|_{E_{0}}^{1-\theta}\|U\|_{E_{1}}^{\theta}, \quad \forall U \in E_{1}$;

4. Sejam $\left(Y_{0}, Y_{1}\right)$ um par de interpolação e $B \in \mathcal{L}\left(E_{j}, Y_{j}\right), j=0,1$, então $B \in \mathcal{L}\left(E_{\theta}, Y_{\theta}\right)$. E ainda, se $\|B x\|_{Y_{0}} \leq c_{0}\|x\|_{E_{0}} e\|B x\|_{Y_{1}} \leq c_{1}\|x\|_{E_{1}}$, então $\|B x\|_{Y_{\theta}} \leq c_{\theta}\|x\|_{E_{\theta}}$, onde $c_{\theta}=$ $c_{0}^{1-\theta} c_{1}^{\theta}$.

Como mencionado, vamos definir o espaço de Bessel por interpolação

Teorema 2.2.15 (Triebel (1978), Teorema 1, pág. 317) Sejam $\Omega \subset \mathbb{R}^{n}$ um domínio limitado suave, $1<p<\infty$ e $0 \leq s_{0} \leq s_{1}<\infty$. Então,

$$
H_{p}^{s}(\Omega)=\left[H_{p}^{s_{0}}(\Omega), H_{p}^{s_{1}}(\Omega)\right]_{\theta},
$$

onde $0 \leq \theta \leq 1$ e $s=s_{0}(1-\theta)+\theta s_{1}$. 
Por fim, assim como foi feito para $\mathbb{R}^{n}$, seguem os resultados de imersões e equivalências dos espaços, relacionando os respectivos expoentes:

Teorema 2.2.16 (Triebel (1978), pág.310) Sejam $\Omega \subset \mathbb{R}^{n}$ um domínio arbitrário, $s \in \mathbb{R} e$ $1<p<\infty$. Então:

$$
H_{p}^{s}(\Omega)=W_{p}^{s}(\Omega)
$$

se, e somente se, $s \in \mathbb{Z}$.

Teorema 2.2.17 (Triebel (1978), pág 330-332) Sejam $\Omega \subset \mathbb{R}^{n}$ um domínio limitado suave $e$ $1<p<\infty$. Então:

1. Se $s \in\left(-\infty, \frac{1}{p}\right]$, então $\mathcal{D}(\Omega)$ é denso em $H_{p}^{s}(\Omega)$.

2. Se $\frac{1}{p}-1<s<\frac{1}{p} e \frac{1}{p}+\frac{1}{q}=1$, então $\left(H_{p}^{s}(\Omega)\right)^{\prime}=H_{q}^{-s}(\Omega)$.

Teorema 2.2.18 (Triebel (1978), pág 328) Sejam $\Omega \subset \mathbb{R}^{n}$ um domínio arbitário, $1<p, q<$

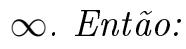

- Se $l \geq 0$ e $s>l+\frac{n}{p}$, então $H_{p}^{s}(\Omega) \hookrightarrow C^{l}(\Omega)$.

Se além disso, $\Omega \subset \mathbb{R}^{n}$ é limitado, temos

- Se $s-\frac{n}{p} \geq t-\frac{n}{q}$ e $s \geq t$, então $H_{p}^{s}(\Omega) \hookrightarrow H_{q}^{t}(\Omega)$;

- Se $s>t, p \geq q$ e $s>\frac{1}{p}$, então $H_{p}^{s}(\Omega) \hookrightarrow H_{q}^{t}(\Omega)$ é compacta.

\subsubsection{O operador traço em $H_{p}^{s}(\Omega)$}

Seja $u: \Omega \rightarrow \mathbb{K}, \mathbb{K}=\mathbb{R}$ ou $\mathbb{K}=\mathbb{C}$, denotaremos o operador traço $\gamma(u)=\left.u\right|_{\partial \Omega}$, isto é, a restrição de $u$ em $x \in \partial \Omega$. Em particular, vamos estudar o operador traço definido em

$$
\gamma: H_{p}^{s}(\Omega) \rightarrow L^{r}(\partial \Omega)
$$

Lema 2.2.19 (Triebel (1978), pág. 330) Seja $\Omega \subset \mathbb{R}^{n}$ limitado e suave. Se $1<p<\infty, s>\frac{1}{p}$ e $s-\frac{n}{p} \geq-\frac{(n-1)}{r}$, então o operador traço

$$
\gamma: H_{p}^{s}(\Omega) \rightarrow L^{r}(\partial \Omega)
$$

está bem definido e é um operador linear contínuo e sobrejetor.

\subsection{Teoria de semigrupos}

Quando tenta-se estabelecer propriedades das soluções de problemas parabólicos, em geral, é necessário fazer um estudo das propriedades espectrais dos operadores lineares associados aos problemas parabólicos. Além disso, é necessário a teoria de semigrupos. Os resultados desta seção podem ser encontrados em Brezis (2010); Carvalho (2001); Henry (1981) e Yosida (1980).

Segundo o autor Carvalho (2001), um semigrupo é um caso particular de um sistema dinâmico que consiste numa família de operadores lineares $\{T(t): t \geq 0\}$ definidos num espaço de Banach $E$, de dimensão infinita ou finita, que satisfazem algumas propriedades. Em toda esta seção, $E$ denota um espaço de Banach e $I$ denota o operador identidade em $E$.

Definição 2.3.1 (Semigrupos) Um semigrupo de operadores lineares em E é uma família $\{T(t): t \geq 0\} \subset \mathcal{L}(E)$ tal que 
1. $T(0)=I$, onde $I$ é a identidade em $E$;

2. $T(t+s)=T(t) T(s)$ para todo $t, s \geq 0$;

Adicionalmente, se

- $\|T(t)-I\|_{\mathcal{L}(E)} \rightarrow 0$, quando $t \rightarrow 0^{+}$, o semigrupo é dito uniformemente contínuo;

- $\|T(t) x-x\|_{E} \rightarrow 0$, quando $t \rightarrow 0^{+}, \forall x \in E$, o semigrupo é dito fortemente contínuo.

Todo semigrupo fortemente contínuo possui uma limitação exponencial, que é dada a seguir.

Teorema 2.3.2 (Carvalho (2001), Teorema 1.1.1.) Seja $\{T(t): t \geq 0\} \subset \mathcal{L}(E)$ um semigrupo fortemente contínuo. Então, existem constantes $\beta \in \mathbb{R}$ e $M \geq 1$ tais que

$$
\|T(t)\|_{\mathcal{L}(E)} \leq M e^{\beta t}, \quad \forall t \geq 0 .
$$

Para qualquer $l>0$ é possível escolher $\beta \geq \frac{1}{l} \log \|T(l)\|_{\mathcal{L}(E)}$ e então escolher $M$.

Definição 2.3.3 (Gerador infinitesimal) $S e\{T(t): t \geq 0\} \subset \mathcal{L}(E)$ é um semigrupo fortemente contínuo de operadores lineares, seu gerador infinitesimal é um operador definido por $A: D(A) \subset$ $E \rightarrow E$, onde

$$
D(A)=\left\{x \in E: \lim _{t \rightarrow 0^{+}} \frac{T(t) x-x}{t} \quad \text { existe }\right\} \quad \text { e } \quad A x=\lim _{t \rightarrow 0^{+}} \frac{T(t) x-x}{t} .
$$

O teorema a seguir estabelece alguns resultados importantes de semigrupos fortemente contínuos.

Teorema 2.3.4 (Carvalho (2001), Teoremas 1.1.2. e 1.1.3.) Sejam $\{T(t): t \geq 0\} \subset \mathcal{L}(E)$ um semigrupo fortemente contínuo e A o seu gerador infinitesimal. Então seguem as afirmativas:

1. Para qualquer $x \in E, t \mapsto T(t) x$ é contínuo para $t \geq 0$;

2. A aplicação $t \mapsto\|T(t)\|_{\mathcal{L}(E)}$ é semicontínua inferiormente e, portanto, mensurável;

3. $O$ operador $A$ é densamente definido e fechado. E ainda, para todo $x \in D(A)$, a aplicação $t \mapsto T(t) x$ é continuamente diferenciável $e$

$$
\frac{d}{d t} T(t) x=A T(t) x=T(t) A x, \quad t>0 ;
$$

4. $\bigcap_{m \geq 1} D\left(A^{m}\right)$ é denso em $E$;

5. Seja $\beta$ dado pela limitação exponencial no Teorema 2.3.2. Seja $\lambda \in \mathbb{C}$ tal que $\operatorname{Re}(\lambda)>\beta$, então $\lambda \in \rho(A) e$

$$
(\lambda I-A)^{-1} x=\int_{0}^{\infty} e^{-\lambda t} T(t) x d t, \quad \forall x \in E ;
$$

6. Sejam $\{S(t): t \geq 0\} \subset \mathcal{L}(E)$ um semigrupo fortemente continuo e $B$ o seu gerador infinitesimal. Se $A=B$ então $T(t)=S(t), \quad \forall t \geq 0$.

Enunciaremos o teorema de Hille-Yosida:

Teorema 2.3.5 (Carvalho (2001), Teorema 1.2.2.) Seja $A: D(A) \subset E \rightarrow E$ um operador linear. As seguintes afirmativas são equivalentes: 
1. A é o gerador infinitesimal de um semigrupo fortemente contínuo $\{T(t): t \geq 0\} \subset \mathcal{L}(E)$ tal que

$$
\|T(t)\|_{\mathcal{L}(E)} \leq M e^{\beta t}, \quad \forall t \geq 0
$$

2. A é fechado, densamente definido, $(\beta, \infty) \subset \rho(A) e$

$$
\left\|(\lambda I-A)^{-n}\right\|_{\mathcal{L}(E)} \leq \frac{M}{(\lambda-\beta)^{n}}, \quad \forall \operatorname{Re}(\lambda)>\beta \quad \text { e } n=1,2, \ldots
$$

Já foi definido que o semigrupo é uma família de operadores lineares com determinadas propriedades e pelo Teorema 2.3.4 é observado que, se $A$ é o gerador infinitesimal do semigrupo fortemente contínuo $\{T(t): t \geq 0\}$ então vale a relação

$$
\frac{\mathrm{d}}{\mathrm{d} t} T(t) x=A T(t) x=T(t) A x, \quad t>0 .
$$

Da mesma forma que as soluções de Equações Diferenciais Ordinárias (E.D.O.) do tipo,

$$
\frac{d x(t)}{d t}=A x(t)
$$

onde $A$ era uma matriz em dimensão finita, eram dadas por uma fórmula exponencial $e^{A t}$, os semigrupos motivam a ideia de procurar semigrupos fortemente contínuos como soluções de problemas. O resultado a seguir relaciona semigrupos fortemente contínuos, seu gerador infinitesimal e uma fórmula exponencial.

Teorema 2.3.6 (Carvalho (2001), Teorema 1.4.1) Seja $\{T(t): t \geq 0\} \subset \mathcal{L}(E)$ um semigrupo fortemente continuo. Se

$$
A(h) x=\frac{T(h) x-x}{h},
$$

entẫo para todo $x \in E$ temos

$$
T(t) x=\lim _{h \rightarrow 0^{+}} e^{t A(h)} x,
$$

e o limite é uniforme para $t$ em intervalos limitado de $\mathbb{R}$.

Em geral, esse limite será denotado por $T(t) x=e^{A t} x$.

Definição 2.3.7 (Operador setorial) Um operador linear $A \in \mathcal{L}(E)$ é dito setorial, se é fechado, densamente definido e existem $M \geq 1, a \in \mathbb{R}$ e $\phi \in\left(0, \frac{\pi}{2}\right)$ tais que o setor

$$
\Sigma_{a, \phi}=\{\lambda \in \mathbb{C}: \phi \leq|\arg (\lambda-a)| \leq \pi, \quad \lambda \neq a\} \subset \rho(A),
$$

e, além disso,

$$
\left\|(\lambda I-A)^{-1}\right\|_{\mathcal{L}(E)} \leq \frac{M}{|\lambda-a|}, \quad \forall \lambda \in \Sigma_{a, \phi}
$$

Definição 2.3.8 (Semigrupo analítico) Um semigrupo analítico é uma família de operadores lineares contínuos em $E,\{T(t): t \geq 0\} \subset \mathcal{L}(E)$, satisfazendo

1. $T(0)=I, T(t) T(s)=T(t+s)$ para $t, s \geq 0 ;$

2. $T(t) x \rightarrow x$ quando $t \rightarrow 0^{+}$, para cada $x \in E$;

3. $t \rightarrow T(t) x$ é analítica real em $0<t<\infty$, para cada $x \in E$.

Vamos denotar $T(t)=e^{A t}$, onde A é o gerador infinitesimal desse semigrupo. 
Teorema 2.3.9 (Henry (1981), Teorema 1.3.4) Seja $A: D(A) \subset E \rightarrow E$ um operador setorial. Então, $-A$ é o gerador infinitesimal de um semigrupo analítico $\left\{e^{-A t}: t \geq 0\right\}$, onde

$$
e^{-A t}=\frac{1}{2 \pi i} \int_{\Gamma} e^{\lambda t}(\lambda I+A)^{-1} d \lambda,
$$

onde $\Gamma$ é a fronteira em $\rho(-A)$ com arg $(\lambda) \rightarrow \pm \theta$ quando $|\lambda| \rightarrow \infty$ para algum $\theta \in\left(\frac{\pi}{2}, \pi\right)$.

Além disso, $e^{-A t}$ se estende analiticamente em um setor $\{t \neq 0:|\arg (t)|<c\}, c>0$, contendo o eixo real positivo, e se $\operatorname{Re}(\sigma(A))>a$, isto é, $\operatorname{Re}(\lambda)>$ a onde $\lambda \in \sigma(A)$, entẫo para todo $t>0$

$$
\left\|e^{-A t}\right\|_{\mathcal{L}(E)} \leq K e^{-a t} \quad e \quad\left\|A e^{-A t}\right\|_{\mathcal{L}(E)} \leq K t^{-1} e^{-a t}
$$

para alguma constante $K>0$. Por fim,

$$
\frac{d}{d t} e^{-A t}=-A e^{-A t}, \quad t>0
$$

Definição 2.3.10 (Operador positivo) Um operador linear $A \in \mathcal{L}(E)$ é dito tipo positivo com constante $M \geq 1$, se é fechado, densamente definido, $\mathbb{R}^{+} \subset \rho(-A) e$

$$
\left\|(\lambda I+A)^{-1}\right\|_{\mathcal{L}(E)} \leq \frac{M}{(\lambda+1)}, \quad \lambda \in \mathbb{R}^{+} .
$$

Observação 2.3.11 Se A é um operador positivo é possivel construir um setor $\Sigma_{M} \subset \rho(-A), e$ portanto, $-A$ é setorial e define um semigrupo fortemente contínuo, ver Carvalho (2001), pág. 27.

\subsection{Escalas de interpolação e extrapolação}

Os resultados a seguir podem ser vistos em Amann (1995); Arrieta e Carvalho (1999); Brezis (2010) e Triebel (1978) e é uma continuação da teoria de interpolação mencionada na Subseção 2.2.10.

Seja $A_{0}: D\left(A_{0}\right) \subset E_{0} \rightarrow E_{0}$ o gerador infinitesimal de um semigrupo analítico, com $\left(E_{0}, D\left(A_{0}\right)\right)$ um par de interpolação (sob essas hipóteses, $\exists \omega \geq 0$ tal que $\operatorname{Re}\left(\sigma\left(\omega I+A_{0}\right)\right)>0$ ).

Denotemos o espaço $E_{1}=D\left(A_{0}\right)$ induzido pela norma do gráfico, isto é, $\|u\|_{E_{1}}=\left\|\left(\omega I+A_{0}\right) u\right\|_{E_{0}}$, $u \in E_{1}$, e o operador

$$
A_{1}: D\left(A_{1}\right) \subset E_{1} \rightarrow E_{1} .
$$

Note que $E_{1}=D\left(A_{0}\right)$ é um espaço de Banach, pois $A_{0}$ é fechado.

O operador $A_{1}$ é chamado de $E_{1}$-realização do operador $A_{0}$, ou a restrição do operador $A_{0} \mathrm{em}$ $E_{1}$. Indutivamente para $k \in \mathbb{N}$, se os pares $\left(E_{k}, E_{k+1}\right)$ ainda são pares de interpolação é possível definir uma escala de espaços de Banach discreta

- $E_{k+1}=D\left(A_{k}\right)$ induzido pela norma $\left\|\left(\omega I+A_{k}\right) u\right\|_{E_{k}}, u \in E_{k+1}$;

- $A_{k+1}$ é a $E_{k+1}$-realização do operador $A_{0}$;

- $A_{k+1} x=A_{0} x$, para todo $x$ em $E_{k+1}$.

Por fim, utilizando a teoria de interpolação complexa com $0 \leq \theta \leq 1$ e $k \in \mathbb{N}$, define-se

$$
E_{k+\theta}=\left[E_{k}, E_{k+1}\right]_{\theta}
$$

e, consequentemente, uma escala de espaços de Banach contínua e positiva:

$$
\left\{E_{\alpha}: \alpha \in \mathbb{R}^{+}\right\} .
$$

E ainda, $A_{\alpha}: E_{\alpha+1} \subset E_{\alpha} \rightarrow E_{\alpha}$ denota a realização de $A_{0}$ em $E_{\alpha}$. 
Em geral, a realização do operador é necessária para que as aplicações do problema estejam bem definidas abstratamente, contudo, uma escala positiva de espaço de Banach nem sempre satisfaz a necessidade de determinados problemas, pois as aplicações precisam estar em um ambiente maior e menos regular, mesmo que algumas propriedades de regularidade da solução sejam perdida. Dessa forma, sob as hipóteses anteriores, claramente, $\omega \in \rho\left(A_{0}\right)$, e portanto, o operador resolvente tem inversa bem definida, contínua (limitada) e densamenta definida. Logo, essas propriedades motivam definir

$$
X=E_{1} \quad \text { induzido pela norma } \quad\left\|\left(\omega I+A_{1}\right)^{-1}(\cdot)\right\|_{E_{1}} .
$$

Como $X$ é um subespaço linear denso de $E_{0}$ com normas equivalentes, pode-se resgatar as informações de $A_{0}, E_{0}$, então, de fato, essa ideia não apenas motiva, $E_{0}$ é o completamento de $X$ e $A_{0}$ uma extensão contínua de $A_{1}$ em $E_{0}$.

Assim, intuitivamente, essa construção sugere que, sob certas hipóteses, $A_{0}$ admite uma extensão contínua $A_{-1}$ em algum espaço maior $E_{-1}$, isto é, $A_{-1}=A_{0}$ em $E_{0}$, e assim define-se $E_{-1}=E_{0}$ com a norma do gráfico $\left\|\left(\omega I+A_{0}\right)^{-1}(\cdot)\right\|_{E_{0}}$. Desde que os espaços continuem satisfazendo a definição de par de interpolação, também de forma indutiva e usando o interpolador, é possível estender a escala contínua de espaços de Banach para valores negativos de $\alpha$

$$
\left\{E_{\alpha}: \alpha \in \mathbb{R}\right\}
$$

Para uma melhor descrição desses espaços de potências negativas utilizaremos a escala dual.

Considere que os espaços de Banach utilizados a partir de agora são reflexivos, isto é, existe uma bijeção canônica bem definida entre o espaço e o seu bidual. Seja $A_{0}: D\left(A_{0}\right) \subset E_{0} \rightarrow E_{0}$ um operador com as mesmas hipóteses anteriores e $A_{0}^{\prime}: D\left(A_{0}^{\prime}\right) \subset\left(E_{0}\right)^{\prime} \rightarrow\left(E_{0}\right)^{\prime}$ o operador dual de $A_{0}$.

De forma análoga, é possível construir uma escala de espaços de Banach relacionados ao operador $A_{0}^{\prime},\left\{\left(E_{\alpha}\right)^{\prime}: \alpha \in[-k, \infty)\right\}$. Para construir a parte negativa da escala contínua utilizaremos o espaço dual dos espaços interpolados.

Teorema 2.4.1 (Triebel (1978), pág.72) Seja $\left(E_{k}, E_{k+1}\right), k \in \mathbb{N}$, um par de interpolação, onde $E_{k} \cap E_{k+1}$ é denso tanto em $E_{k}$ quanto em $E_{k+1}$. Se $E_{k}$ ou $E_{k+1}$ é reflexivo, então

$$
\left[E_{k}, E_{k+1}\right]_{\theta}^{\prime}=\left[E_{k+1}^{\prime}, E_{k}^{\prime}\right]_{\theta}, \quad 0 \leq \theta \leq 1 .
$$

Assim, conseguimos uma melhor identificação dessa escala contínua definindo

$$
E_{-(k+\theta)}=\left[E_{k}, E_{k+1}\right]_{\theta}^{\prime}=\left[E_{k+1}^{\prime}, E_{k}^{\prime}\right]_{\theta}, \quad 0 \leq \theta \leq 1 .
$$

Os resultados abaixo condensam essa discussão:

Teorema 2.4.2 (Amann (1995), Teorema 1.4.12) Sejam $E_{0}$ um espaço de Banach reflexivo $e$ $A_{0}$ um operador tipo positivo em $E_{0}$. Então, a escala de espaços de Banach gerados por $\left(E_{0}, A_{0}\right)$ :

$$
\left\{\left(E_{\alpha}, A_{\alpha}\right): \alpha \in \mathbb{R}\right\}
$$

e sua escala dual

$$
\left\{\left(E_{\alpha}^{\prime}, A_{\alpha}^{\prime}\right): \alpha \in \mathbb{R}\right\}
$$

são escalas continuamente e densamente definidas satisfazendo as desigualdades de interpolação. Mais ainda,

$$
E_{-\alpha}=\left(E_{\alpha}\right)^{\prime} \quad \text { e } \quad A_{-\alpha}=\left(A_{\alpha}\right)^{\prime}, \quad \forall \alpha \in \mathbb{R}
$$

com respeito a dualidade induzida por $\langle\cdot, \cdot\rangle$.

Uma escala de ordem $m>0$ de $\left(E_{0}, A_{0}\right)$ será denotada por:

$$
\left\{\left(E_{\alpha}, A_{\alpha}\right): \alpha \in[-m, \infty)\right\} .
$$


Teorema 2.4.3 (Amann (1995), Teorema 2.1.3) Assuma as hipóteses do Teorema 2.4.2 e seja $\left(E_{\alpha}, A_{\alpha}\right)$ a escala de espaços de Banach de ordem $m$ gerada por $\left(E_{0}, A_{0}\right)$.

Suponha que $A_{0}$ é o gerador infinitesimal de um semigrupo analítico e existe um $n \in \mathbb{N}$ tal que

- $D\left(A_{0}^{k}\right)=E_{k}, \quad 0 \leq k \leq n$;

- $D\left(\left(A_{0}^{\prime}\right)^{k}\right)=E_{k}^{\prime}, \quad 0 \leq k \leq m$.

Então, $A_{\alpha}$ é um gerador infinitesimal de um semigrupo analítico com $-m \leq \alpha \leq n-1, n \in \mathbb{N}$, e $\rho\left(A_{\alpha}\right)=\rho\left(A_{0}\right)$.

\subsection{Existência e unicidade de soluções de equações parabólicas}

Nesta seção veremos alguns resultados abstratos sobre existência e unicidade de soluções para problemas parabólicos. Os resultados desta seção podem ser encontrados em Carvalho et al. (1997); Czaja (2002) e Henry (1981).

Considere $\Omega \subset \mathbb{R}^{n}$ um domínio limitado e suave. Seja $L$ um operador linear diferencial de segunda ordem:

$$
L u(x)=\sum_{|\alpha| \leq 2} a_{\alpha}(x) D^{\alpha} u(x), \quad x \in \Omega,
$$

onde $\alpha$ é um multi-índice e $a_{\alpha}(x), u(x)$ são regulares o suficiente. Dizemos que $L$ é um operador elíptico se existe uma constante $d>0$ tal que

$$
\sum_{|\alpha|=2} a_{\alpha}(x) x^{\alpha} \geq d\|x\|_{\mathbb{R}^{n}}^{2}, \quad x \in \Omega
$$

Introduzimos o problema parabólico não linear associado ao operador elíptico $L$ :

$$
\begin{cases}\frac{\partial u}{\partial t}(t, x)+L u(t, x)=f(x, u(t, x)), & (t, x) \in(0, \infty) \times \Omega \\ B u(t, x)=g(x, u(t, x)), & (t, x) \in(0, \infty) \times \partial \Omega \\ u(0, x)=u_{0}(x), & x \in \bar{\Omega}\end{cases}
$$

onde definimos o operador de condição de contorno $B$ da seguinte forma: se $B=\frac{\partial}{\partial \vec{n}}$ dizemos que a condição de contorno é de Von Neumann, se $B=I$ dizemos que a condição de contorno é de Dirichlet e se $B=\frac{\partial}{\partial \vec{n}}+V_{0}(x) I$ dizemos que a condição de contorno é de Robin, com a função $V_{0}$ satisfazendo certas condições, ou então, podemos ver a condição de Robin como uma condição de Von Neumann com uma pertubação $V_{0}(x)$.

Em específico, neste trabalho, em (1.4) temos

$$
L=L^{0}=-\Delta+\mu I \quad \text { e } \quad B=\frac{\partial}{\partial \vec{n}}+V_{0}(x) I .
$$

E ainda, no caso de (1.1) temos uma família de operadores perturbados

$$
L^{\epsilon}=-\Delta+\mu I+\frac{1}{\epsilon} \chi_{\omega_{\epsilon}}(x) V_{\epsilon}(x)=L^{0}+\frac{1}{\epsilon} \chi_{\omega_{\epsilon}}(x) V_{\epsilon}(x) \quad \text { e } \quad B=\frac{\partial}{\partial \vec{n}},
$$

com $0<\epsilon \leq \epsilon_{0}$.

Com o objetivo de estudar o problema parabólico (2.1), olhamos o mesmo como uma equação de evolução em um espaço de Banach. Veremos alguns resultados a seguir relacionados a forma abstrata do problema (2.1).

Assuma que $A$ é um operador setorial em um espaço de Hilbert $E \operatorname{com} \operatorname{Re}(\sigma(A))>0$. Nós podemos definir as potências fracionárias $A^{\alpha}$ de $A$, e os espaços de potências fracionárias $E^{\alpha}=$ $D\left(A^{\alpha}\right)$ induzido com a norma do gráfico $\|x\|_{E^{\alpha}}=\left\|A^{\alpha} x\right\|_{E}, \alpha \geq 0$. Agora, definimos $E^{-\alpha}=\left(E^{\alpha}\right)^{\prime}$ 
para $\alpha>0$. E ainda, as realizações de $A$ nesta escala, isto é, $A^{\alpha}: E^{\alpha+1} \subset E^{\alpha} \rightarrow E^{\alpha}, \alpha \in \mathbb{R}$, são setoriais. Com abuso de notação, usaremos $A$ para todas as realizações do operador $A$. Como $E$ é um espaço de Hilbert, a escala de espaços $\left\{\left(E_{\alpha}, A_{\alpha}\right): \alpha \in \mathbb{R}\right\}$ construída por interpolação na Seção 2.4 coincide com os espaços de potências fracionárias. Para mais detalhes ver Amann (1995); Carvalho et al. (1997) e Henry (1981).

O teorema a seguir garante existência local, unicidade e dependência contínua das soluções

Teorema 2.5.1 Sejam A um operador setorial, $\operatorname{Re}(\sigma(A))>0$, com e $h: E^{\alpha} \rightarrow E^{\beta}$ localmente Lipschitziana, isto é, para todo limitado $B \subset E^{\alpha}$, existe uma constante $k_{B}>0$ tal que

$$
\|h(u)-h(v)\|_{E^{\beta}} \leq k_{B}\|u-v\|_{E^{\alpha}}, \quad \forall u, v \in E^{\alpha},
$$

onde $0 \leq \alpha-\beta \leq 1$. Então, dado $u_{0} \in E^{\alpha}$, existe $T=T\left(u_{0}\right)>0$ tal que o problema parabólico abstrato

$$
\left\{\begin{array}{l}
\frac{d u}{d t}+A u=h(u), \quad t>0 \\
u(0)=u_{0}
\end{array}\right.
$$

tem um única solução $u:[0, T) \rightarrow E^{\alpha}$ contínua dada pela fórmula da variação das constantes

$$
u(t)=e^{-A t} u_{0}+\int_{0}^{t} e^{-A(t-s)} h(u(s)) d s, \quad t \in[0, T),
$$

onde $e^{-A t}$ denota o semigrupo analítico gerado por $-A$. E ainda, essa solução depende continuamente do dado inicial $u_{0}$.

Demonstração: Ver Teorema 6.2.1 Carvalho (2001), Teorema 3.1 em Carvalho et al. (1997) e Teorema 3.3.3 em Henry (1981).

E ainda, temos o seguinte resultado

Teorema 2.5.2 Sejam A, h e $u_{0}$ como no Teorema 2.5.1 e $u:[0, T) \rightarrow E^{\alpha}$ a solução maximal de (2.2). Se $T<\infty$ então

$$
\lim _{t \rightarrow T^{-}} \sup \frac{\|h(u(t))\|_{E^{\beta}}}{1+\|u(t)\|_{E^{\alpha}}}=\infty .
$$

Demonstração: Ver Teorema 6.2.2 Carvalho (2001), Teorema 3.1 em Carvalho et al. (1997) ou Teorema 3.3.4 em Henry (1981).

Como consequência temos a existência global de soluções.

Corolário 2.5.3 Sejam $A, h$ e $u_{0}$ como no Teorema 2.5.1 e $u:[0, T) \rightarrow E^{\alpha}$ a solução maximal de (2.2). Suponha que $h(B)$ é um subconjunto limitado em $E^{\beta}$, para todo conjunto limitado $B \subset E^{\alpha}$. Se $T<\infty$ então

$$
\lim _{t \rightarrow T^{-}}\|u(t)\|_{E^{\alpha}}=\infty
$$

Consequentemente, se $\|u(t)\|_{E^{\alpha}}<\infty$, para t em intervalos finitos, então a solução $u(t)$ de (2.2) existe para todo $t \geq 0$.

Demonstração: Consequência do Corolário 3.3.5 em Henry (1981). 
Observação 2.5.4 No caso específico dos nossos problemas parabólicos (1.1) e (1.4), no Capítulo 3, usaremos as condiçôes de crescimento (H1) e de sinal (H2), para verificar as condiçôes dos teoremas abstratos e garantir que os problemas parabólicos (1.1) e (1.4) estâa bem postos para todo $t \geq 0$.

Por fim, é importante destacar que ao trabalhar com equações parabólicas é necessário fazer algumas estimativas sobre a fórmula da variação das constantes, fato que torna indispensável o uso da desigualdade de Gronwall. Essa versão da desigualdade de Gronwall pode ser encontrada com mais detalhes no Lema 6.2.1 de Carvalho (2001).

Lema 2.5.5 Assuma que $a, b \geq 0,0 \leq \alpha, \beta<1$ e $u:[0, T] \rightarrow \mathbb{R}$ é integrável com

$$
0 \leq u(t) \leq a t^{-\alpha}+b \int_{0}^{t}(t-s)^{-\beta} u(s) d s,
$$

quase sempre em $(0, T)$. Então, existe uma constante $K=K(b, \beta, T)>0$ tal que

$$
u(t) \leq \frac{K}{1-\alpha} a t^{-\alpha}
$$

quase sempre em $(0, T)$.

\subsection{Teoria de atratores}

Os resultados desta seção podem ser encontrados em Carvalho (2012); Hale (1988) e Temam (1988).

Sejam $E$ um espaço de Banach com norma $\|\cdot\|_{E},\{T(t): t \geq 0\} \subset \mathcal{C}(E)$ um semigrupo e $B \subset E$ um subconjunto, definimos:

- Para cada $t \geq 0$, a imagem de $B$ sob $T(t)$,

$$
T(t) B=\{T(t) x: x \in B\} ;
$$

- A órbita positiva de $B$,

$$
\gamma^{+}(B)=\bigcup_{t \geq 0} T(t) B
$$

- A órbita parcial de $B$ entre dois número $0 \leq t_{0}<t_{1}<\infty$,

$$
\gamma_{\left[t_{0}, t_{1}\right]}^{+}(B)=\bigcup_{t_{0} \leq t \leq t_{1}} T(t) B
$$

- A órbita positiva de $T(t) B$,

$$
\gamma_{t}^{+}(B)=\bigcup_{s \geq 0} T(t+s) B=\bigcup_{s \geq t} T(s) B
$$

- O conjunto $\omega$-limite de $B$,

$$
\omega(B)=\bigcap_{t \geq 0} \overline{\gamma_{t}^{+}(B)}
$$

Com base nas notações anteriores, temos

Definição 2.6.1 Um semigrupo $\{T(t): t \geq 0\} \subset \mathcal{C}(E)$ é dito eventualmente limitado se para cada $B \subset E$ limitado, existe $t_{B} \geq 0$ tal que $\gamma_{t_{B}}^{+}(B)$ é limitada. Se $\gamma^{+}(B)$ for limitada sempre que $B$ for limitado iremos dizer que o semigrupo é limitado. 
Além disso, dados dois subconjuntos $A, B \subset E$, denotaremos por $d_{E}(A, B)$ a semi distância de Hausdorff entre $A$ e $B$

$$
d_{E}(A, B)=\sup _{x \in A} \mathrm{~d}(x, B)=\sup _{x \in A} \inf _{y \in B}\|x-y\|_{E} .
$$

A seguir definimos as noções de atração, absorção e invariância sob a ação do semigrupo $\{T(t)$ : $t \geq 0\}$

Definição 2.6.2 Sejam $A, B \subset E$. Diremos que $A$ atrai $B$ sob a ação de um semigrupo $\{T(t): t \geq$ $0\} \subset \mathcal{C}(E)$, se

$$
\lim _{t \rightarrow \infty} d_{E}(T(t) B, A)=0 .
$$

Se existir um $t_{0}=t_{0}(B)>0$ tal que $T(t) B \subset A$ para todo $t \geq t_{0}$, diremos que $A$ absorve $B$. Também iremos dizer que um subconjunto $A \subset E$ é invariante por $T(t)$, se $T(t) A=A$ para todo $t \geq 0$.

Observação 2.6.3 Se A absorve $B$, então $A$ atrai B. Contudo, a recíproca não é válida.

Observação 2.6.4 Um conjunto unitário formado por um ponto de equilíbrio $u^{*}$ de $\{T(t): t \geq 0\}$, isto é, um ponto $u^{*} \in E$ tal que $T(t) u^{*}=u^{*}$, para todo $t \geq 0$, é invariante por $\{T(t): t \geq 0\}$.

Assim, podemos definir os atratores globais

Definição 2.6.5 (Atratores globais) Um conjunto $\mathcal{A} \subset E$ é chamado de atrator global para $\{T(t): t \geq 0\} \subset \mathcal{C}(E)$ se é compacto, invariante, maximal e atrai subconjuntos limitados de $E$ sob a ação de $T(t)$.

Observação 2.6.6 Note que pela definição de atração de conjuntos, se um atrator existe, ele é único. De fato, se $\mathcal{A}$ e $\mathcal{A}^{*}$ são atratores globais para $\{T(t): t \geq 0\}$, então

$$
d_{E}\left(\mathcal{A}, \mathcal{A}^{*}\right)=d_{E}\left(T(t) \mathcal{A}, \mathcal{A}^{*}\right) \rightarrow 0, \quad \text { quando } \quad t \rightarrow \infty,
$$

e assim, $\mathcal{A} \subset \mathcal{A}^{*}$. Analogamente, $\mathcal{A}^{*} \subset \mathcal{A}$.

Definição 2.6.7 Um semigrupo $\{T(t): t \geq 0\} \subset \mathcal{C}(E)$ é dito:

1. assintoticamente compacto se para qualquer subconjunto $B \subset E$ fechado, limitado e não vazio, para o qual $T(t) B \subset B$, para todo $t \geq 0$, existe um conjunto compacto $J \subset B$ que atrai $B$;

2. condicionalmente eventualmente compacto se dado $B \subset E$ limitado e invariante, existe $t_{B}>0$ tal que $\overline{T\left(t_{B}\right) B}$ é compacto;

3. eventualmente compacto se dado $B \subset$ E limitado, existe $t_{B}>0$ tal que $\overline{T\left(t_{B}\right) B}$ é compacto;

4. ponto (limitado ou compacto) dissipativo se existir um subconjunto limitado $B \subset E$ que atrai pontos (subconjuntos limitado ou subconjuntos compactos) de E.

Teorema 2.6.8 (Carvalho (2012), Teorema 1.0.22) $S e\{T(t): t \geq 0\} \subset \mathcal{C}(E)$ é um semigrupo condicionalmente eventualmente compacto, então é assintoticamente compacto.

Com as definições postas é possível enunciar o teorema que garante a existência de um atrator global para um semigrupo.

Teorema 2.6.9 (Hale (1988), Teorema 3.4.6.) $S e\{T(t): t \geq 0\} \subset \mathcal{C}(E)$ é um semigrupo eventualmente limitado, ponto dissipativo e assintoticamente compacto, então $T(t)$ possui um atrator global $\mathcal{A}$. 
Além de estudar a existência de atratores, vamos tentar entender a relação entre os atratores de cada problema e a convergência da família de atratores, isto é, estamos também interessados em saber se os atratores se comportam continuamente com relação a algum parâmetro. Para tanto, precisamos da seguinte definição

Definição 2.6.10 (Continuidade da família de atratores) Sejam $\Lambda$ um espaço topológico e $\left\{\mathcal{A}_{\lambda}\right\}_{\lambda \in \Lambda}$ uma familia de subconjuntos de $E$.

1. Diremos que $\left\{\mathcal{A}_{\lambda}\right\}_{\lambda \in \Lambda}$ é semicontínua superiormente em $\lambda_{0} \in \Lambda$ se

$$
d_{E}\left(\mathcal{A}_{\lambda}, \mathcal{A}_{\lambda_{0}}\right) \rightarrow 0 \quad \text { quando } \quad \lambda \rightarrow \lambda_{0}
$$

2. Diremos que $\left\{\mathcal{A}_{\lambda}\right\}_{\lambda \in \Lambda}$ é semicontinua inferiormente em $\lambda_{0} \in \Lambda$ se

$$
d_{E}\left(\mathcal{A}_{\lambda_{0}}, \mathcal{A}_{\lambda}\right) \rightarrow 0 \quad \text { quando } \quad \lambda \rightarrow \lambda_{0}
$$

3. Diremos que $\left\{\mathcal{A}_{\lambda}\right\}_{\lambda \in \Lambda}$ é contínua em $\lambda_{0} \in \Lambda$ se é semicontinua superiormente e inferiormente em $\lambda_{0}$.

Para provar as semicontinuidades superior e inferior, podemos usar o seguinte resultado

Lema 2.6.11 (Carvalho (2012), Lema 1.2.2.) Sejam $\Lambda$ um espaço topológico e $\left\{\mathcal{A}_{\lambda}\right\}_{\lambda \in \Lambda}$ uma familia de subconjuntos de E.

1. Se qualquer sequência $\left\{x_{\lambda_{n}}\right\}_{n \in \mathbb{N}}$ com $x_{\lambda_{n}} \in \mathcal{A}_{\lambda_{n}}, \lambda_{n} \rightarrow \lambda_{0}$ quando $n \rightarrow \infty$, tem uma subsequência convergente com limite pertencendo a $\mathcal{A}_{\lambda_{0}}$, entâo $\left\{\mathcal{A}_{\lambda}\right\}_{\lambda \in \Lambda}$ é semicontínua superiormente em $\lambda_{0} \in \Lambda$;

2. Se $\mathcal{A}_{\lambda_{0}}$ é compacto e para qualquer $x \in \mathcal{A}_{\lambda_{0}}$, existe uma sequência $\left\{x_{\lambda_{n}}\right\}_{n \in \mathbb{N}}$ com $x_{\lambda_{n}} \in \mathcal{A}_{\lambda_{n}}$, $\lambda_{n} \rightarrow \lambda_{0}$ quando $n \rightarrow \infty$, que converge para $x$, então $\left\{\mathcal{A}_{\lambda}\right\}_{\lambda \in \Lambda}$ é semicontínua inferiormente em $\lambda_{0} \in \Lambda$.

\subsection{Integrais concentradas}

Uma vez que estamos interessados no comportamento das soluções do problema parabólico com termos concentrados, nesta seção, faremos um resumo com os principais resultados sobre integrais concentradas que serão utilizados posteriormente. Os resultados expostos aqui podem ser encontrados com mais detalhes em Arrieta et al. (2008) e Jiménes-Casas e Rodríguez-Bernal (2011).

Para $\delta \geq 0$ definimos a fronteira interior paralela $\Gamma_{\delta}=\{x-\delta \vec{n}(x): x \in \partial \Omega\}$, onde $\vec{n}(x)$ é vetor normal unitário exterior em $x \in \partial \Omega$. Em particular, $\Gamma_{0}=\partial \Omega$. Assim é possível reescrever a faixa de largura $\epsilon$ e base $\partial \Omega$ como:

$$
\omega_{\epsilon}=\{x-\delta \vec{n}(x): x \in \partial \Omega \quad \text { e } \quad \delta \in[0, \epsilon)\}=\bigcup_{0 \leq \delta<\epsilon} \Gamma_{\delta},
$$

para $\epsilon$ suficientemente pequeno, digamos $0<\epsilon \leq \epsilon_{0}$.

Portanto, para qualquer função integrável, $f$, definida em $\omega_{\epsilon}, 0<\epsilon \leq \epsilon_{0}$, segue que

$$
\int_{\omega_{\epsilon}} f \mathrm{~d} x=\int_{0}^{\epsilon} \int_{\Gamma_{\delta}} f \mathrm{~d} S_{\delta} \mathrm{d} \delta
$$

onde $\mathrm{d} S_{\delta}$ é a medida associada a superfície $\Gamma_{\delta}$.

Para $\epsilon_{0}$ suficientemente pequeno, se $0<\delta<\epsilon_{0}$ e $\Omega_{\delta}=\Omega \backslash \overline{\omega_{\delta}}$, então podemos construir um difeomorfismo $C^{2}, \tau_{\delta}: \bar{\Omega} \rightarrow \overline{\Omega_{\delta}}$, da seguinte forma

$$
\tau_{\delta}(x)=\left\{\begin{array}{lll}
x, & \text { se } & \mathrm{d}(x, \partial \Omega) \geq \epsilon_{0} \\
z-\psi_{\delta}(\sigma) \vec{n}(z), & \text { se } \quad x=z-\sigma \vec{n}(z), \quad \sigma \in\left[0, \epsilon_{0}\right)
\end{array}\right.
$$


onde $\psi_{\delta}:\left[0, \epsilon_{0}\right] \rightarrow\left[\delta, \epsilon_{0}\right]$ é uma função crescente $C^{2}$ satisfazendo $\psi_{\delta}(0)=\delta$ e $\psi_{\delta}\left(\epsilon_{0}\right)=\epsilon_{0}$ e

$$
d(x, \partial \Omega)=\inf _{y \in \partial \Omega}\|x-y\|_{\mathbb{R}^{n}}, \quad x \in \bar{\Omega} .
$$

Com essa construção, temos que a aplicação $\delta \mapsto \tau_{\delta} \in C^{2}(\bar{\Omega})$ é contínua para $\delta \in\left[0, \epsilon_{0}\right]$ e

$$
\left\|\tau_{\delta}-I\right\|_{C^{2}(\bar{\Omega})} \rightarrow 0 \quad \text { quando } \quad \delta \rightarrow 0 .
$$

E ainda, $\tau_{\delta}$ é um difeormorfismo de classe $C^{2}$ entre $\partial \Omega$ e $\Gamma_{\delta}$, e como $\psi_{\delta}(0)=\delta$, então, $\tau_{\delta}(x)=$ $x-\delta \vec{n}(x)$, para $x \in \partial \Omega$.

Realizando a mudança de variável na integral (2.3), com o difeormorfismo $\tau_{\delta}$, para $f$ definida em $\omega_{\epsilon}$ segue que

$$
\int_{\omega_{\epsilon}} f \mathrm{~d} x=\int_{0}^{\epsilon} \int_{\partial \Omega} \gamma\left(f\left(\tau_{\delta}(x)\right)\right) J\left(\tau_{\delta}(x)\right) \mathrm{d} S \mathrm{~d} \delta,
$$

onde denotaremos por $\mathrm{d} S$ a medida associada a superfície $\Gamma_{0}=\partial \Omega, J\left(\tau_{\delta}(x)\right)$ o jacobiano da transformação $\tau_{\delta}$ e $\gamma$ o operador traço.

Por fim, temos que existem constantes $0<J_{1} \leq J_{2}$ tais que

$J_{1} \leq J\left(\tau_{\delta}(x)\right) \leq J_{2} \quad$ e $\quad\left\|J\left(\tau_{\delta}\right)-1\right\|_{L^{\infty}(\partial \Omega)} \rightarrow 0 \quad$ quando $\quad \delta \rightarrow 0, \quad \forall x \in \partial \Omega \quad$ e $\quad \delta \in\left[0, \epsilon_{0}\right]$.

Com base nessa construção, vamos enunciar alguns resultados e dar uma ideia de suas respectivas demonstrações.

Lema 2.7.1 (Arrieta et al. (2008), Lema 2.1.) Seja $v \in H_{p}^{s}(\Omega) \operatorname{com} \frac{1}{p}<s \leq 2$ e $s-\frac{n}{p} \geq-\frac{n-1}{q}$. Então, para $\epsilon_{0}$ suficientemente pequeno temos

1. A aplicação

$$
\delta \mapsto \int_{\Gamma_{\delta}}|v(x)|^{q} d S_{\delta}, \quad \delta \in\left[0, \epsilon_{0}\right]
$$

é contínua;

2. Existe uma constante $C>0$ independente de $\epsilon$ e $v$ tal que, para todo $\epsilon \leq \epsilon_{0}$,

$$
\sup _{\delta \in[0, \epsilon)}\|v\|_{L^{q}\left(\Gamma_{\delta}\right)} \leq C\|v\|_{H_{p}^{s}(\Omega)} .
$$

3. $\int_{\omega_{\epsilon}}|v(x)|^{q} d x=\int_{0}^{\epsilon} \int_{\Gamma_{\delta}}|v(x)|^{q} d S_{\delta} d \delta$.

Em particular,

$$
\frac{1}{\epsilon} \int_{\omega_{\epsilon}}|v(x)|^{q} d x \leq C\|v\|_{H_{p}^{s}(\Omega)}^{q} \quad e \quad \lim _{\epsilon \rightarrow 0} \frac{1}{\epsilon} \int_{\omega_{\epsilon}}|v(x)|^{q} d x=\int_{\partial \Omega}|\gamma(v(x))|^{q} d S .
$$

\section{Demonstração:}

Para essa prova precisaremos da construção do difeormorfismo $\tau_{\delta}$, portanto, considere as notações acima, principalmente a mudança de variável

$$
\int_{\Gamma_{\delta}}|v(x)|^{q} \mathrm{~d} S_{\delta}=\int_{\partial \Omega}\left|\gamma\left(v\left(\tau_{\delta}(x)\right)\right)\right|^{q} J\left(\tau_{\delta}(x)\right) \mathrm{d} S .
$$

1. Para concluir essa etapa da demonstração é suficiente que para qualquer $\delta_{0} \in\left[0, \epsilon_{0}\right], \epsilon_{0}$ fixado, temos

$$
\lim _{\delta \rightarrow \delta_{0}} \int_{\Gamma_{\delta}}|v(x)|^{q} \mathrm{~d} S_{\delta}-\int_{\Gamma_{\delta_{0}}}|v(x)|^{q} \mathrm{~d} S_{\delta_{0}}=0
$$


ou ainda,

$$
\left.\left|\int_{\partial \Omega}\right| \gamma\left(v\left(\tau_{\delta}(x)\right)\right)\right|^{q} J\left(\tau_{\delta}(x)\right) \mathrm{d} S-\int_{\partial \Omega}\left|\gamma\left(v\left(\tau_{\delta_{0}}(x)\right)\right)\right|^{q} J\left(\tau_{\delta_{0}}(x)\right) \mathrm{d} S \mid \rightarrow 0, \quad \text { quando } \quad \delta \rightarrow \delta_{0} .
$$

Primeiro, vamos observar que a restrição $\tau_{\delta}: \partial \Omega \rightarrow \Gamma_{\delta}, \delta \in\left[0, \epsilon_{0}\right]$ ainda é um difeomorfismo de classe $C^{2}$ e, portanto, seu Jacobiano, $J\left(\tau_{\delta}(x)\right)$, computado utilizando as primeiras derivadas de $\tau_{\delta}$, ainda é pelo menos $C^{1}$ em $x$. E ainda, a aplicação $\delta \mapsto J\left(\tau_{\delta}(\cdot)\right) \in C^{1}$ é uma função contínua.

Segundo, pela definição de $\Gamma_{\delta}$ e como $\partial \Omega$ é $C^{2}$, sabemos que existem constantes $0<J_{1} \leq J_{2}$, tais que

$$
J_{1} \leq J\left(\tau_{\delta}(x)\right) \leq J_{2}, \quad \forall \delta \in\left[0, \epsilon_{0}\right] \quad \text { e } \quad \forall x \in \partial \Omega .
$$

Além disso, se $v \in H_{p}^{s}(\Omega)$ pela continuidade de $\tau_{\delta}$ obtemos que $v\left(\tau_{\delta}\right) \in H_{p}^{s}(\Omega)$ e $\delta \mapsto v\left(\tau_{\delta}\right)$ ainda é uma aplicação contínua. Por último, dado $f \in L^{q}(\partial \Omega)$ definimos a norma com peso $J\left(\tau_{\delta_{0}}\right)$ e $\delta_{0} \in\left[0, \epsilon_{0}\right]$,

$$
\|f\|_{L_{\delta_{0}}^{q}(\partial \Omega)}=\int_{\partial \Omega}|f(x)|^{q} J\left(\tau_{\delta_{0}}(x)\right) \mathrm{d} S .
$$

Note que essa norma é equivalente a norma usual de $L^{q}(\partial \Omega)$, pois

$$
J_{1} \int_{\partial \Omega}|f(x)|^{q} \mathrm{~d} S \leq \int_{\partial \Omega}|f(x)|^{q} J\left(\tau_{\delta_{0}}(x)\right) \mathrm{d} S \leq J_{2} \int_{\partial \Omega}|f(x)|^{q} \mathrm{~d} S .
$$

Pela equivalência das normas e pelo Lema 2.2.19), obtemos que o operador traço

$$
\gamma: H_{p}^{s}(\Omega) \rightarrow L_{\delta_{0}}^{q}(\partial \Omega)
$$

é continuo, linear e sobrejetor para cada $\delta_{0} \in\left[0, \epsilon_{0}\right]$ fixo.

Em particular, para cada $\delta_{0} \in\left[0, \epsilon_{0}\right]$ fixo, a aplicação

$$
\delta \mapsto \int_{\partial \Omega}\left|\gamma\left(v\left(\tau_{\delta}(x)\right)\right)\right|^{q} J\left(\tau_{\delta_{0}}(x)\right) \mathrm{d} S
$$

é contínua.

Somando e subtraindo $\int_{\partial \Omega}\left|\gamma\left(v\left(\tau_{\delta}\right)\right)\right|^{q} J\left(\tau_{\delta_{0}}(x)\right) \mathrm{d} S$, obtemos que

$$
\begin{aligned}
& \left.\left|\int_{\partial \Omega}\right| \gamma\left(v\left(\tau_{\delta}(x)\right)\right)\right|^{q} J\left(\tau_{\delta}(x)\right) \mathrm{d} S-\int_{\partial \Omega}\left|\gamma\left(v\left(\tau_{\delta_{0}}(x)\right)\right)\right|{ }^{q} J\left(\tau_{\delta_{0}}(x)\right) \mathrm{d} S \mid \\
& \leq\left.\left|\int_{\partial \Omega}\right| \gamma\left(v\left(\tau_{\delta}(x)\right)\right)\right|^{q} J\left(\tau_{\delta_{0}}(x)\right) \mathrm{d} S-\int_{\partial \Omega}\left|\gamma\left(v\left(\tau_{\delta_{0}}(x)\right)\right)\right|^{q} J\left(\tau_{\delta_{0}}(x)\right) \mathrm{d} S \mid \\
& +\int_{\partial \Omega}\left|\gamma\left(v\left(\tau_{\delta}(x)\right)\right)\right|^{q}\left|J\left(\tau_{\delta}(x)\right)-J\left(\tau_{\delta_{0}}(x)\right)\right| \mathrm{d} S \rightarrow 0,
\end{aligned}
$$

quando $\delta \rightarrow \delta_{0}$, onde usamos a continuidade em (2.5) e a continuidade da aplicação $\delta \mapsto J\left(\tau_{\delta}\right)$.

2. A demonstração desse resultado foge do escopo do nosso trabalho, portanto, omitiremos maiores detalhes, os quais podem ser encontrados em Arrieta et al. (2008), Lema 2.1.

3. A primeira parte do item 3. segue direto do Teorema de Fubbini.

Em particular, da continuidade no item 1 e do item 2, obtemos

$$
\frac{1}{\epsilon} \int_{\omega_{\epsilon}}|v(x)|^{q} \mathrm{~d} x=\frac{1}{\epsilon} \int_{0}^{\epsilon} \int_{\Gamma_{\delta}}|v(x)|^{q} \mathrm{~d} S_{\delta} \mathrm{d} \delta \leq \sup _{\delta \in[0, \epsilon)}\|v\|_{L^{q}\left(\Gamma_{\delta}\right)} \leq C\|v\|_{H_{p}^{s}(\Omega)} .
$$

Por fim, da primeira parte do item 3. sabemos que

$$
\left.\left|\frac{1}{\epsilon} \int_{\omega_{\epsilon}}\right| v(x)\right|^{q} \mathrm{~d} x-\int_{\partial \Omega}|\gamma(v(x))|^{q} \mathrm{~d} S|=| \frac{1}{\epsilon} \int_{0}^{\epsilon}\left(\int_{\Gamma_{\delta}}|v(x)|{ }^{q} \mathrm{~d} S_{\delta}-\int_{\partial \Omega}|\gamma(v(x))|^{q} \mathrm{~d} S\right) \mathrm{d} \delta \mid
$$




$$
\leq\left.\sup _{\delta \in[0, \epsilon)}\left|\int_{\Gamma_{\delta}}\right| v(x)\right|^{q} \mathrm{~d} S_{\delta}-\int_{\partial \Omega}|\gamma(v(x))|^{q} \mathrm{~d} S \mid .
$$

Agora, como $\Gamma_{0}=\partial \Omega$, pela continuidade do item 1,

$$
\left.\lim _{\delta \rightarrow 0}\left|\int_{\Gamma_{\delta}}\right| v(x)\right|^{q} \mathrm{~d} x-\int_{\partial \Omega}|v(x)|^{q} \mathrm{~d} S \mid=0
$$

quando $\delta \rightarrow 0$.

O lema a seguir é enunciado e demonstrado em Jiménes-Casas e Rodríguez-Bernal (2011).

Lema 2.7.2 (Jiménes-Casas e Rodríguez-Bernal (2011), Lema 5.1) Sob as notações acima, se $\epsilon_{0}>0$ é suficientemente pequeno e $0 \leq \delta<\epsilon_{0}$, então para todo $1<q<\infty$, existe uma constante $M>0$ independente de $\delta$ tal que para toda $v \in H_{q}^{1}(\Omega)$, temos

$$
\left\|\gamma\left(v\left(\tau_{\delta}\right)-v\right)\right\|_{L^{q}(\partial \Omega)} \leq M \delta^{1-\frac{1}{q}}\|\nabla v\|_{L^{q}\left(\omega_{\delta}\right)} .
$$

\section{Demonstração:}

Primeiramente, seja $v \in C^{1}(\bar{\Omega})$ e considere a função $\phi(t)=v(x-t \delta \vec{n}(x)) \operatorname{com} t \in[0,1]$ e $x \in \partial \Omega$. Então, para todo $x \in \partial \Omega$ e $1<q<\infty$, pelo Teorema Fundamental do Cálculo e a integração sob sinal, obtemos

$$
\left|v\left(\tau_{\delta}(x)\right)-v(x)\right|^{q}=|\phi(1)-\phi(0)|^{q}=\left|\int_{0}^{1} \phi^{\prime}(t) \mathrm{d} t\right|^{q}
$$

e

$$
\left|\int_{0}^{1} \phi^{\prime}(t) \mathrm{d} t\right|^{q} \leq \int_{0}^{1}|\nabla v(x-t \delta \vec{n}(x))|{ }^{q}|\delta \vec{n}(x)|^{q} \mathrm{~d} t \leq \delta^{q} \int_{0}^{1}|\nabla v(x-t \delta \vec{n}(x))|^{q} \mathrm{~d} t .
$$

Logo,

$$
\int_{\partial \Omega}\left|v\left(\tau_{\delta}(x)\right)-v(x)\right|^{q} \mathrm{~d} S \leq \delta^{q} \int_{0}^{1} \int_{\partial \Omega}|\nabla v(x-t \delta \vec{n}(x))|^{q} \mathrm{~d} S \mathrm{~d} t .
$$

Multiplicando o argumento da integral por $\left|\frac{J\left(\tau_{t \delta}(x)\right)}{J\left(\tau_{t \delta}(x)\right)}\right|$ e utilizando que $0<J_{1} \leq J\left(\tau_{t \delta}(x)\right) \leq J_{2}$, $t \in[0,1]$ e $\delta \in\left[0, \epsilon_{0}\right]$, obtemos

$$
\int_{\partial \Omega}\left|v\left(\tau_{\delta}(x)\right)-v(x)\right|^{q} \mathrm{~d} S \leq \frac{\delta^{q}}{J_{1}} \int_{0}^{1} \int_{\partial \Omega}|\nabla v(x-t \delta \vec{n}(x))|^{q}\left|J\left(\tau_{t \delta}(x)\right)\right| \mathrm{d} S \mathrm{~d} t .
$$

Utilizando a mudança de variável com $\tau_{t \delta}$ e a outra mudança $s=t \delta$

$$
\int_{\partial \Omega}\left|v\left(\tau_{\delta}(x)\right)-v(x)\right|^{q} \mathrm{~d} S \leq \frac{\delta^{q}}{J_{1}} \int_{0}^{1} \int_{\Gamma_{t \delta}}|\nabla v(x)|^{q} \mathrm{~d} S_{t \delta} \mathrm{d} t=\frac{\delta^{q-1}}{J_{1}} \int_{0}^{\delta} \int_{\Gamma_{s}}|\nabla v(x)|{ }^{q} \mathrm{~d} S_{s} \mathrm{~d} s .
$$

Portanto, do item 3 do Lema 2.7.1,

$$
\left\|v\left(\tau_{\delta}\right)-v\right\|_{L^{q}(\partial \Omega)}^{q} \leq \frac{\delta^{q-1}}{J_{1}}\|\nabla \phi\|_{L^{q}\left(\omega_{\delta}\right)}^{q} .
$$

Para $v \in H_{q}^{1}(\Omega)$ a prova segue por argumentos de densidade e da continuidade do operador traço $\gamma: H_{q}^{1}(\Omega) \rightarrow L^{q}(\partial \Omega)$. 


\subsection{Problemas elípticos com concentração}

Um problema elíptico em $\Omega$ é uma equação diferencial parcial associada a um operador diferencial elíptico com determinadas condições sobre a fronteira de $\Omega$. Como estamos interessados em estudar o comportamento das soluções, quando $\epsilon \rightarrow 0$, do problema parabólico com termos concentrados (1.1), então precisaremos entender o que ocorre com as soluções de problemas elípticos com termos concentrados, e isto foi analisado em Arrieta et al. (2008).

Mais especificadamente, nesta seção trataremos de um problema elíptico importante na conclusão da limitação uniforme das soluções e da existência dos atratores globais dos problemas parabólicos que estamos estudando. Utilizando a mesma notação do Capítulo 1 e considerando as funções $C, D, E$ e $F$ como em (H2), vamos analisar o limite das soluções do seguinte problema elíptico

$$
\begin{cases}L^{\epsilon} \Phi^{\epsilon}(x)=C(x) \Phi^{\epsilon}(x)+D(x)+\frac{1}{\epsilon} \chi_{\omega_{\epsilon}}(x)\left[E(x) \Phi^{\epsilon}(x)+F(x)\right], & x \in \Omega \\ \frac{\partial \Phi^{\epsilon}}{\partial \vec{n}}(x)=0, & x \in \partial \Omega\end{cases}
$$

onde

$$
L^{\epsilon} \Phi^{\epsilon}(x)=-\Delta \Phi^{\epsilon}(x)+\mu \Phi^{\epsilon}(x)+\frac{1}{\epsilon} \chi_{\omega_{\epsilon}}(x) V_{\epsilon}(x) \Phi^{\epsilon}(x),
$$

com $\mu>0$ e $0<\epsilon \leq \epsilon_{0}$. Veremos que o problema limite de (2.6) é dado por

$$
\begin{cases}L^{0} \Phi^{0}(x)=C(x) \Phi^{0}(x)+D(x), & x \in \Omega \\ \frac{\partial \Phi^{0}}{\partial \vec{n}}(x)+V_{0}(x) \Phi^{0}(x)=E(x) \Phi^{0}(x)+F(x), & x \in \partial \Omega\end{cases}
$$

onde

$$
L^{0} \Phi^{0}(x)=-\Delta \Phi^{0}(x)+\mu \Phi^{0}(x) .
$$

Teorema 2.8.1 Suponha que as hipóteses (H2) e (P) valem e que $\mu>0$ é suficientemente grande. Então, para cada $\epsilon \in\left(0, \epsilon_{0}\right]$, existe uma única solução fraca $\Phi^{\epsilon} \in H^{1}(\Omega)$ do problema concentrado (2.6) e uma única solução fraca $\Phi^{0} \in H^{1}(\Omega)$ do problema limite (2.7) que satisfazem:

1. $\Phi^{\epsilon} \rightarrow \Phi^{0}$ em $H^{1}(\Omega)$, quando $\epsilon \rightarrow 0$;

2. Para cada $0 \leq \epsilon \leq \epsilon_{0}, \Phi^{\epsilon} \in L^{\infty}(\Omega)$ e existe $K>0$ independente de $\epsilon$ tal que

$$
\left\|\Phi^{\epsilon}\right\|_{L^{\infty}(\Omega)} \leq K
$$

3. $\Phi^{\epsilon} \rightarrow \Phi^{0}$ em $L^{\infty}(\Omega)$, quando $\epsilon \rightarrow 0$.

\section{Demonstração:}

1. Teorema 4.1. em Arrieta et al. (2008);

2. Teorema 4.4. em Arrieta et al. (2008);

3. Teorema 4.6. em Arrieta et al. (2008).

É importante também estudar os autovalores do operador elíptico para obter limitações uniformes das soluções dos problemas parabólicos e entender o comportamente da família de atratores. Dessa forma, considere os seguintes problemas de autovalores 


$$
\begin{cases}-\Delta \phi_{i}^{\epsilon}(x)+[\mu-C(x)] \phi_{i}^{\epsilon}(x)+\frac{1}{\epsilon} \chi_{\omega_{\epsilon}}(x)\left[V_{\epsilon}(x)-E(x)\right] \phi_{i}^{\epsilon}(x)=\lambda_{i}^{\epsilon} \phi_{i}^{\epsilon}(x), & x \in \Omega \\ \frac{\partial \phi_{i}^{\epsilon}}{\partial \vec{n}}(x)=0, & x \in \partial \Omega\end{cases}
$$

e

$$
\begin{cases}-\Delta \phi_{i}^{0}(x)+[\mu-C(x)] \phi_{i}^{0}(x)=\lambda_{i}^{0} \phi_{i}^{0}(x), & x \in \Omega \\ \frac{\partial \phi_{i}^{0}}{\partial \vec{n}}(x)+V_{0}(x) \phi_{i}^{0}(x)=E(x) \phi_{i}^{0}(x), & x \in \partial \Omega\end{cases}
$$

com $i=1,2, \ldots$. Como consequência do Teorema 2.8.1 temos o seguinte resultado

Corolário 2.8.2 Suponha que as hipóteses (H2) e (P) valem e que $\mu>0$ é suficientemente grande. Então, para cada $i=1,2,3, \ldots$,

1. $\lambda_{i}^{\epsilon} \rightarrow \lambda_{i}^{0}$, quando $\epsilon \rightarrow 0$;

2. $\phi_{i}^{\epsilon} \rightarrow \phi_{i}^{0}$ em $H^{1}(\Omega)$ e $L^{\infty}(\Omega)$, quando $\epsilon \rightarrow 0$.

Demonstração: Ver Corolário 4.2. e Observação 4.3 em Arrieta et al. (2008).

Observação 2.8.3 Denotemos por $\lambda_{1}$ o primeiro autovalor do problema elíptico (1.3), isto é, do seguinte problema

$$
\begin{cases}-\Delta \phi_{1}^{0}(x)+[\mu-C(x)] \phi_{1}^{0}(x)=\lambda_{1} \phi_{1}^{0}(x), & x \in \Omega \\ \frac{\partial \phi_{1}^{0}}{\partial \vec{n}}(x)+V_{0}(x) \phi_{1}^{0}(x)=E(x) \phi_{1}^{0}(x), & x \in \partial \Omega\end{cases}
$$

e, para cada $0<\epsilon \leq \epsilon_{0}$, denotemos por $\lambda_{1}^{\epsilon}$ o primeiro autovalor do problema

$$
\left\{\begin{array}{ll}
-\Delta \phi_{1}^{\epsilon}(x)+[\mu-C(x)] \phi_{1}^{\epsilon}(x)+\frac{1}{\epsilon} \chi_{\omega_{\epsilon}}(x)\left[V_{\epsilon}(x)-E(x)\right] \phi_{1}^{\epsilon}(x)=\lambda_{1}^{\epsilon} \phi_{1}^{\epsilon}(x), & x \in \Omega \\
\frac{\partial \phi_{1}^{\epsilon}}{\partial \vec{n}}(x)=0, & x \in \partial \Omega
\end{array} .\right.
$$

Em particular, do Corolário 2.8.2, temos que $\lambda_{1}^{\epsilon} \rightarrow \lambda_{1}$, quando $\epsilon \rightarrow 0$. Logo, se vale a condição de dissipatividade (H3), então $\lambda_{1}>0$ e, para $\epsilon_{0}$ suficientemente pequeno, obtemos $\lambda_{1}^{\epsilon}>0,0<\epsilon \leq \epsilon_{0}$. 


\section{Capítulo 3}

\section{Existência, unicidade e limitação uniforme das soluções}

Antes de realizarmos qualquer um dos nossos objetivos neste trabalho, primeiramente, precisamos verificar se existem soluções para os problemas (1.1) e (1.4) e se essas soluções são únicas. Para tanto, o método que iremos utilizar é a teoria de semigrupos, vamos escrever (1.1) e (1.4) de uma forma abstrata. Uma vez que temos uma fórmula abstrata para os problemas (1.1) e (1.4), usaremos os resultados abstratos da Seção 2.5 para mostrar que os problemas estão bem postos, isto é, as soluções fracas existem, são únicas e dependem continuamente do dado inicial. A seguir, daremos uma ideia de algumas maneiras de definirmos a formulação abstrata de problemas parabólicos.

Para definirmos os problemas abstratos associados a (1.1) e (1.4) e mostrarmos que são bem postos, uma maneira é usarmos o espaço $L^{2}(\Omega)$ e as propriedades dos operadores elípticos com condições de fronteira (Neumann ou Robin) homogêneas associados a (1.1) e (1.4) neste espaço. Uma vez que isso é feito, precisamos escolher o espaço de fase, se escolhermos o $L^{2}(\Omega)$ então as aplicações abstratas associadas as não linearidades que aparecem nos problemas (1.1) e (1.4) podem não estar bem definidas de $L^{2}(\Omega)$ em $L^{2}(\Omega)$ e, consequentemente, os problemas abstratos podem não estar bem definidos no espaço ambiente $L^{2}(\Omega)$. Logo, precisamos diminuir o espaço de fase para abordar um maior número de não linearidades, e assim o uso do espaço $H^{1}(\Omega)$ como o espaço de fase, torna-se natural. Como estamos usando os operadores elípticos com condições de fronteira homogêneas, para definirmos somente a equação abstrata associada a (1.1), podemos escolher o $L^{2}(\Omega)$ como espaço ambiente. Entretanto, uma vez que (1.4) possui termos não lineares sobre a fronteira, então para definirmos a equação abstrata associada a (1.4), precisamos escolher um espaço de Bessel de ordem negativa, isto é, $H^{-\alpha}(\Omega), \frac{1}{2}<\alpha \leq 1$, para introduzir esses termos não lineares na equação abstrata. Neste caso, precisamos ainda impor algumas restrições no crescimento e sinal, dadas por (H1) e (H2), das não linearidades nos problemas (1.1) e (1.4), para garantirmos que os problemas abstratos estão bem definidos no espaço ambiente $H^{-\alpha}(\Omega)$ e que temos existência e unicidade de soluções para esses problemas, bem como impor condições de dissipação, (H3), para que o semigrupo não linear associado às soluções seja limitado e dissipativo. Essas restrições ficam mais rigorosas quando a dimensão $n$ do espaço aumenta. Neste trabalho, vamos utilizar essa abordagem do problema e a mesma pode ser encontrada em Arrieta e Carvalho (1999); Carvalho et al. (1997); Jiménes-Casas e Rodríguez-Bernal (2009, 2011).

Uma outra maneira é definirmos o operador elíptico em $L^{p}(\Omega), 1<p<\infty$, e usarmos como espaços de fase, os espaços das potências fracionárias $E^{\alpha}, \alpha \geq 0$, e esses espaços possuem imersão no espaço das funções contínuas $C^{0}(\Omega)$, para alguns valores de $\alpha$, o que é uma grande vantagem neste caso, pois não precisamos impor restrições sobre o crescimento das não linearidades. Note que a exigência de imersão contínua do $H^{1}(\Omega)$ em $C^{0}(\Omega)$ restringe o estudo para o caso unidimensional $n=1$. Além disso, os espaços de potências fracionárias estão relacionados com os espaços de Bessel, e essa relação é dada usando a teoria de inteporlação. Mais detalhes podem ser encontrados em Henry (1981); Oliva e Pereira (2002). 
A seguir veremos a formulação abstrata dos problemas (1.1) e (1.4), bem como o estudo da existência, unicidade e limitação uniforme das soluções desses problemas.

\subsection{Formulação abstrata dos problemas parabólicos}

Nesta seção vamos introduzir uma formulação abstrata para o problema parabólico concentrado (1.1) e o seu candidato a problema limite (1.4). Vamos introduzir uma fórmula variacional e uma solução num sentido mais fraco para esses problemas.

Inicialmente, suponhamos que os problemas (1.1) e (1.4) possuam soluções clássicas $u^{\epsilon}$ e $u^{0}$, respectivamente, e seja $\phi \in \mathcal{C}^{\infty}(\bar{\Omega})$. Multiplicando os problemas (1.1) e (1.4) por $\phi$, integrando em $\Omega$ e usando a Fórmula de Green, obtemos

$$
\begin{array}{r}
\int_{\Omega}\left[u_{t}^{\epsilon}(t, x) \phi(x)+\mu u^{\epsilon}(t, x) \phi(x)+\nabla u^{\epsilon}(t, x) \nabla \phi(x)\right] \mathrm{d} x+\frac{1}{\epsilon} \int_{\omega_{\epsilon}} V_{\epsilon}(x) u^{\epsilon}(t, x) \phi(x) \mathrm{d} x= \\
\int_{\Omega} f\left(x, u^{\epsilon}(t, x)\right) \phi(x) \mathrm{d} x+\frac{1}{\epsilon} \int_{\omega_{\epsilon}} g\left(x, u^{\epsilon}(t, x)\right) \phi(x) \mathrm{d} x,
\end{array}
$$

para $0<\epsilon \leq \epsilon_{0}$, e

$$
\begin{array}{r}
\int_{\Omega}\left[u_{t}^{0}(t, x)+\mu u^{0}(t, x) \phi(x)+\nabla u^{0}(t, x) \nabla \phi(x)\right] \mathrm{d} x+\int_{\partial \Omega} V_{0}(x) u^{0}(t, x) \phi(x) \mathrm{d} S= \\
\int_{\Omega} f\left(x, u^{0}(t, x)\right) \phi(x) \mathrm{d} x+\int_{\partial \Omega} g\left(x, u^{0}(t, x)\right) \phi(x) \mathrm{d} S .
\end{array}
$$

Estamos interessados em encontrar soluções fracas de (1.1) e (1.4), isto é, funções $u^{\epsilon}$ e $u^{0}$ que satisfazem os problemas variacionais (3.1) e (3.2), respectivamente, em espaços adequados menos regulares. Além disso, em (3.2) temos integrais na fronteira $\partial \Omega$ do domínio $\Omega$, logo as funções envolvidas precisam ter traço. Dessa forma, como nosso espaço de fase será o $H^{1}(\Omega)$ temos a seguinte definição (Ver Definição 3.1 em Arrieta e Carvalho (1999)).

Definição 3.1.1 (Solução fraca) Para $u_{0} \in H^{1}(\Omega)$ as soluçôes fracas de (1.1) e (1.4) são funcôes $u^{\epsilon} \in C\left(\left[0, T^{\epsilon}\right), H^{1}(\Omega)\right), 0<\epsilon \leq \epsilon_{0}$, e $u^{0} \in C\left(\left[0, T^{0}\right), H^{1}(\Omega)\right)$, respectivamente, para algum $T^{\epsilon}, T^{0}>$ 0 , tais que as fórmulas variacionais (3.1) e (3.2) são satisfeitas para toda $\phi \in H^{1}(\Omega)$.

Observação 3.1.2 Usamos as seguintes notações para as soluções

$$
\begin{aligned}
& u^{\epsilon}:\left[0, T^{\epsilon}\right) \rightarrow H^{1}(\Omega) \\
& t \quad \mapsto u^{\epsilon}(t): \quad \Omega \rightarrow \mathbb{R} \\
& x \mapsto u^{\epsilon}(t)(x)=u^{\epsilon}(t, x)
\end{aligned}
$$

com $0 \leq \epsilon \leq \epsilon_{0}$.

Quando necessário, explicitaremos a dependência da condição inicial, isto é, $u^{\epsilon}\left(t, x, u_{0}\right)$.

Agora, vamos escrever as formulações abstratas para (1.1) e (1.4), ou ainda, para (3.1) e (3.2). Seja $E_{0}=L^{2}(\Omega)$ e considere o operador linear elíptico $A: D(A) \subset L^{2}(\Omega) \rightarrow L^{2}(\Omega)$ dado por

$$
A u=-\Delta u+\mu u, \quad \forall u \in D(A),
$$

onde

$$
D(A)=\left\{u \in H^{2}(\Omega): \frac{\partial u}{\partial \vec{n}}=0 \quad \text { sobre } \quad \partial \Omega\right\} .
$$

Considere também o operador linear elíptico $A^{0}: D\left(A^{0}\right) \subset L^{2}(\Omega) \rightarrow L^{2}(\Omega)$ dado por

$$
A^{0} u=-\Delta u+\mu u, \quad \forall u \in D\left(A^{0}\right),
$$


onde

$$
D\left(A^{0}\right)=\left\{u \in H^{2}(\Omega): \frac{\partial u}{\partial \vec{n}}+V_{0}(x) u=0 \quad \text { sobre } \quad \partial \Omega\right\} .
$$

Temos o seguinte resultado, cuja prova pode ser encontrada nos Exemplos 5.1.5, 5.1.6 e 5.1.7 de Czaja (2002) ou Proposição 2.1 em Carvalho et al. (1997).

Proposição 3.1.3 (Resultados sobre o Laplaciano) Para algum $\mu>0$ e $V_{0} \in L^{\rho}(\partial \Omega), \rho>$ $n-1$, os operadores $A$ e $A^{0}$ definidos em (3.3) e (3.4), respectivamente, são fechados, densamente definidos, setoriais, positivos e com resolvente compacto em $L^{2}(\Omega)$.

Como $E_{0}=L^{2}(\Omega)$ é reflexivo e tomando $E_{1}=D(A)$ ou $E_{1}=D\left(A^{0}\right)$, podemos usar as técnicas de interpolação-extrapolação vistas na Seção 2.4 para os pares $\left(E_{0}, D(A)\right)$ e $\left(E_{0}, D\left(A^{0}\right)\right)$, ou ainda as técnicas da Seção 2.5. Pelo Teorema 2.4.2 construímos uma escala de espaços de Banach $\left\{\left(E_{\alpha}, A_{\alpha}\right): \alpha \in \mathbb{R}\right\}$ ou $\left\{\left(E_{\alpha}, A_{\alpha}^{0}\right): \alpha \in \mathbb{R}\right\}$. Em particular, para $\alpha \geq 0$,

$$
E_{\alpha} \hookrightarrow H^{2 \alpha}(\Omega)
$$

E ainda,

$$
E_{\frac{1}{2}}=H^{1}(\Omega), \quad E_{-\frac{1}{2}}=H^{-1}(\Omega) \quad \text { e } \quad E_{0}=L^{2}(\Omega),
$$

onde estamos definindo $H^{-1}(\Omega):=\left(H^{1}(\Omega)\right)^{\prime}$. Pelo Teorema 2.4.3, os operadores $A$ e $A^{0}$ podem ser estendidos nesta escala de tal maneira que suas realizações $A_{\alpha}, A_{\alpha}^{0}: E_{\alpha+1} \subset E_{\alpha} \rightarrow E_{\alpha}$ são também operadores fechados, densamente definidos, setoriais, positivos e com resolventes compactos, e em particular, $-A_{\alpha}$ e $-A_{\alpha}^{0}$ geram semigrupos analíticos $\left\{e^{-A_{\alpha} t}: t \geq 0\right\}$ e $\left\{e^{-A_{\alpha}^{0} t}: t \geq 0\right\}$ em $E_{\alpha}$. E ainda, para $\alpha=-\frac{1}{2}$, temos que os operadores $A_{-\frac{1}{2}}, A_{-\frac{1}{2}}^{0}: E_{\frac{1}{2}} \subset E_{-\frac{1}{2}} \rightarrow E_{-\frac{1}{2}}$, são dados por

$$
\left\langle A_{-\frac{1}{2}} u, \phi\right\rangle=\int_{\Omega} \nabla u(x) \nabla \phi(x) \mathrm{d} x+\int_{\Omega} \mu u(x) \phi(x) \mathrm{d} x, \quad \forall u, \phi \in H^{1}(\Omega),
$$

e

$$
\left\langle A_{-\frac{1}{2}}^{0} u, \phi\right\rangle=\int_{\Omega} \nabla u(x) \nabla \phi(x) \mathrm{d} x+\int_{\Omega} \mu u(x) \phi(x) \mathrm{d} x+\int_{\partial \Omega} V_{0}(x) u(x) \phi(x) \mathrm{d} S, \quad \forall u, \phi \in H^{1}(\Omega) .
$$

Mais detalhes podem ser encontrados em Arrieta e Carvalho (1999) e na Proposição 2.1. de Carvalho et al. (1997) .

Para cada $0<\epsilon \leq \epsilon_{0}$, definimos o operador linear $A^{\epsilon}: D\left(A^{\epsilon}\right) \subset L^{2}(\Omega) \rightarrow L^{2}(\Omega)$ da seguinte forma

$$
A^{\epsilon} u=A u+\frac{1}{\epsilon} \chi_{\omega_{\epsilon}}(x) V_{\epsilon}(x) u, \quad \forall u \in D\left(A^{\epsilon}\right),
$$

onde $D\left(A^{\epsilon}\right)=D(A)$.

Note que, para cada $0<\epsilon \leq \epsilon_{0}, A^{\epsilon}$ é uma perturbação do operador $A$, e usando resultados de perturbação (Teorema 1.4.8 em Henry (1981)), temos o seguinte resultado, cuja prova pode ser encontrada na Proposição 2.2 em Carvalho et al. (1997), no Teorema 2.2 em Jiménes-Casas e Rodríguez-Bernal (2011) e Lema 2.1 e Teorema 2.2 em Rodríguez-Bernal (2011).

Proposição 3.1.4 Suponha que existe $K_{1}>0$ independente de $\epsilon$ tal que

$$
\frac{1}{\epsilon} \int_{\omega_{\epsilon}}\left|V_{\epsilon}(x)\right|^{\rho} d x \leq K_{1},
$$

para algum $\rho>n-1$. Se $\mu>0$ e $0<\epsilon \leq \epsilon_{0}$, o operador $A^{\epsilon}$ definido em (3.5) é fechado, densamente definido, setorial, positivo e com resolvente compacto em $L^{2}(\Omega)$. Como antes, existe uma escala de espaços de Banach $\left\{\left(E_{\alpha}, A_{\alpha}^{\epsilon}\right): \alpha \in \mathbb{R}\right\}$, com as realizações $A_{\alpha}^{\epsilon}$ fechadas, densamente definidas, 
setoriais, positivas e com resolvente compacto em $E_{\alpha}$. Em particular, $-A_{\alpha}^{\epsilon}$ gera um semigrupo analítico $\left\{e^{-A_{\alpha}^{\epsilon} t}: t \geq 0\right\}$ em $E_{\alpha}$. E ainda, se $\alpha=-\frac{1}{2}, A_{-\frac{1}{2}}^{\epsilon}: E_{\frac{1}{2}} \subset E_{-\frac{1}{2}} \rightarrow E_{-\frac{1}{2}}$ é dado por

$$
\left\langle A_{-\frac{1}{2}}^{\epsilon} u, \phi\right\rangle=\int_{\Omega} \nabla u(x) \nabla \phi(x) d x+\int_{\Omega} \mu u(x) \phi(x) d x+\frac{1}{\epsilon} \int_{\omega_{\epsilon}} V_{\epsilon}(x) u(x) \phi(x) d x, \quad \forall u, \phi \in H^{1}(\Omega) .
$$

Por abuso de notação, omitiremos o $\alpha \in \mathbb{R}$ nas realizações dos operadores $A_{\alpha}^{0}$ e $A_{\alpha}^{\epsilon}, 0<\epsilon \leq \epsilon_{0}$, identificaremos todas as realizações e escreveremos somente $A^{0}$ e $A^{\epsilon}$. Além disso, temos o seguinte resultado para o semigrupo analítico $\left\{e^{-A^{\epsilon} t}: t \geq 0\right\}, 0 \leq \epsilon \leq \epsilon_{0}$, gerado pelas realizações, onde estamos denotando $H^{-\alpha}(\Omega):=\left(H^{\alpha}(\Omega)\right)^{\prime}, \frac{1}{2}<\alpha \leq 1$.

A estimativa de semigrupo enunciada a seguir pode ser encontrada em Jiménes-Casas e Rodríguez-Bernal (2011), Proposição 2.4. e Observação 2.7.1.

Proposição 3.1.5 Assuma que as funções $C$ e E são como na hipótese (H2), a hipótese (P) é satisfeita e seja $\lambda_{1}$ o primeiro autovalor do problema elíptico (1.3). Então, para $\epsilon_{0}$ suficientemente pequeno e para qualquer $-\beta<\lambda_{1}$ e $\frac{1}{2}<\alpha<1$, temos que existem constantes $M_{1}, M_{2}, M_{3}>0$ independentes de $\epsilon$ tais que

$$
\begin{array}{rlrlrl}
\left\|e^{-A^{\epsilon} t} u_{0}\right\|_{H^{1}(\Omega)} & \leq M_{1} e^{\beta t}\left\|u_{0}\right\|_{H^{1}(\Omega)}, & & t>0 & e & u_{0} \in H^{1}(\Omega) \\
\left\|e^{-A^{\epsilon} t} u_{0}\right\|_{H^{1}(\Omega)} & \leq M_{2} t^{-\left(\frac{1+\alpha}{2}\right)} e^{\beta t}\left\|u_{0}\right\|_{H^{-\alpha}(\Omega)}, & t>0 & e & u_{0} \in H^{-\alpha}(\Omega) . \\
\left\|e^{-A^{\epsilon} t} u_{0}\right\|_{L^{\infty}(\Omega)} & \leq M_{3} t^{-\frac{n}{4}} e^{\beta t}\left\|u_{0}\right\|_{L^{2}(\Omega)}, & & t>0 & e & u_{0} \in L^{2}(\Omega)
\end{array}
$$

Se além disso, a condição de dissipatividade (H3) é satisfeita, então podemos tomar as estimativas

$$
\begin{array}{lllll}
\left\|e^{-A^{\epsilon} t} u_{0}\right\|_{H^{1}(\Omega)} \leq M_{1} e^{-\beta t}\left\|u_{0}\right\|_{H^{1}(\Omega)}, & & t>0 & e & u_{0} \in H^{1}(\Omega) \\
\left\|e^{-A^{\epsilon} t} u_{0}\right\|_{H^{1}(\Omega)} \leq M_{2} t^{-\left(\frac{1+\alpha}{2}\right)} e^{-\beta t}\left\|u_{0}\right\|_{H^{-\alpha}(\Omega)}, & t>0 & e & u_{0} \in H^{-\alpha}(\Omega) . \\
\left\|e^{-A^{\epsilon} t} u_{0}\right\|_{L^{\infty}(\Omega)} \leq M_{3} t^{-\frac{n}{4}} e^{-\beta t}\left\|u_{0}\right\|_{L^{2}(\Omega)}, & & t>0 & e & u_{0} \in L^{2}(\Omega)
\end{array}
$$

para algum $\beta>0$, isto é, o semigrupo $\left\{e^{-A^{\epsilon} t}: t \geq 0\right\}$ possui decaimento exponencial.

Finalmente, definimos as não linearidades abstratas associadas ao problema (1.1) e (1.4). Inicialmente, definimos $F, G^{0}: H^{1}(\Omega) \rightarrow H^{-\alpha}(\Omega)$, com $\frac{1}{2}<\alpha \leq 1$, por

$$
\langle F(u), \phi\rangle=\int_{\Omega} f(x, u(x)) \phi(x) \mathrm{d} x, \quad \forall u \in H^{1}(\Omega) \quad \text { e } \quad \forall \phi \in H^{\alpha}(\Omega),
$$

$\mathrm{e}$

$$
\left\langle G^{0}(u), \phi\right\rangle=\int_{\partial \Omega} \gamma(g(x, u(x))) \gamma(\phi(x)) \mathrm{d} S, \quad \forall u \in H^{1}(\Omega) \quad \text { e } \quad \forall \phi \in H^{\alpha}(\Omega),
$$

onde $\gamma$ é o operador traço dado no Lema 2.2.19. Para cada $0<\epsilon \leq \epsilon_{0}$, definimos $G^{\epsilon}: H^{1}(\Omega) \rightarrow$ $H^{-\alpha}(\Omega), \operatorname{com} \frac{1}{2}<\alpha \leq 1$, por

$$
\left\langle G^{\epsilon}(u), \phi\right\rangle=\frac{1}{\epsilon} \int_{\omega_{\epsilon}} g(x, u(x)) \phi(x) \mathrm{d} x, \quad \forall u \in H^{1}(\Omega) \quad \text { e } \quad \forall \phi \in H^{\alpha}(\Omega) .
$$

Denotando por $h^{0}=F+G^{0}$ e $h^{\epsilon}=F+G^{\epsilon}, 0<\epsilon \leq \epsilon_{0}$, os problemas (1.1) e (1.4) podem ser escritos da seguinte forma abstrata

$$
\left\{\begin{array}{l}
u_{t}^{\epsilon}+A^{\epsilon} u^{\epsilon}=h^{\epsilon}(u), \quad t>0 \\
u^{\epsilon}(0)=u_{0} \in H^{1}(\Omega)
\end{array} .\right.
$$


Agora, vamos provar que as aplicações $h^{\epsilon}, 0 \leq \epsilon \leq \epsilon_{0}$, estão bem definidas.

Proposição 3.1.6 Suponha que $f, g: \bar{\Omega} \times \mathbb{R} \rightarrow \mathbb{R}$ são funções contínuas. Então, para cada $0 \leq \epsilon \leq$ $\epsilon_{0}$, a aplicação $h^{\epsilon}: H^{1}(\Omega) \rightarrow H^{-\alpha}(\Omega)$, com $\frac{1}{2}<\alpha \leq 1$, está bem definida.

\section{Demonstração:}

Como $h^{0}=F+G^{0}$ e $h^{\epsilon}=F+G^{\epsilon}, 0<\epsilon \leq \epsilon_{0}$ é suficiente mostrarmos que $F, G^{0}$ e $G^{\epsilon}$ estão bem definidas em (3.8), (3.9) e (3.10).

1. Para F:

Para cada $u \in H^{1}(\Omega)$, vamos mostrar que $F(u) \in H^{-\alpha}(\Omega)=\left(H^{\alpha}(\Omega)\right)^{\prime}$, isto é,

$$
\begin{array}{rlr}
F(u): H^{\alpha}(\Omega) & \rightarrow & \mathbb{R} \\
\phi & \mapsto & \mapsto F(u), \phi\rangle,
\end{array}
$$

onde

$$
\langle F(u), \phi\rangle=\int_{\Omega} f(x, u(x)) \phi(x) \mathrm{d} x,
$$

é uma aplicação linear contínua.

A linearidade segue da linearidade da integral. Agora, seja $\phi \in H^{\alpha}(\Omega)$, pelo Teorema 2.2.18 temos a seguinte imersão contínua $H^{\alpha}(\Omega) \hookrightarrow L^{2}(\Omega), \frac{1}{2}<\alpha \leq 1$. E ainda, como $u \in H^{1}(\Omega)$ e $f$ é contínua, então a composta $f(\cdot, u(\cdot)) \in H^{1}(\Omega) \hookrightarrow L^{2}(\Omega)$.

Usando a desigualdade de Cauchy-Schwartz, obtemos

$$
\begin{array}{r}
|\langle F(u), \phi\rangle| \leq \int_{\Omega}|f(x, u(x)) \phi(x)| \mathrm{d} x \leq\|f(\cdot, u(\cdot))\|_{L^{2}(\Omega)}\|\phi\|_{L^{2}(\Omega)} \\
\leq c_{1}\|f(\cdot, u(\cdot))\|_{L^{2}(\Omega)}\|\phi\|_{H^{\alpha}(\Omega)} .
\end{array}
$$

Portanto, $F(u) \in H^{-\alpha}(\Omega)$.

2. Para $G^{0}$ :

Para cada $u \in H^{1}(\Omega)$, vamos mostrar que $G^{0}(u) \in H^{-\alpha}(\Omega)=\left(H^{\alpha}(\Omega)\right)^{\prime}$, isto é,

$$
\begin{array}{rlrc}
G^{0}(u): \quad H^{\alpha}(\Omega) & \rightarrow & \mathbb{R} \\
\phi & \mapsto & \left.\mapsto G^{0}(u), \phi\right\rangle,
\end{array}
$$

onde

$$
\left\langle G^{0}(u), \phi\right\rangle=\int_{\partial \Omega} \gamma(g(x, u(x))) \gamma(\phi(x)) \mathrm{d} S,
$$

é uma aplicação linear contínua.

Pelo Lema 2.2.19 temos que o traço $\gamma: H^{\alpha}(\Omega) \hookrightarrow L^{2}(\partial \Omega), \frac{1}{2}<\alpha \leq 1$, é um operador linear contínuo, assim, a linearidade segue da linearidade da integral e do operador traço. E ainda, para cada $\phi \in H^{\alpha}(\Omega)$ temos que $\gamma(\phi) \in L^{2}(\partial \Omega)$. Como $u \in H^{1}(\Omega)$ e $g$ é contínua, então a composta $g(\cdot, u(\cdot)) \in H^{1}(\Omega)$ e $\gamma(g(\cdot, u(\cdot))) \in L^{2}(\partial \Omega)$.

Usando a desigualdade de Cauchy-Schwartz, obtemos

$$
\begin{aligned}
\left|\left\langle G^{0}(u), \phi\right\rangle\right| \leq \int_{\partial \Omega}|\gamma(g(x, u(x))) \gamma(\phi(x))| \mathrm{d} S & \leq\|\gamma(g(\cdot, u(\cdot)))\|_{L^{2}(\partial \Omega)}\|\gamma(\phi)\|_{L^{2}(\partial \Omega)} \\
& \leq c_{2}\|\gamma(g(\cdot, u(\cdot)))\|_{L^{2}(\partial \Omega)}\|\phi\|_{H^{\alpha}(\Omega)} .
\end{aligned}
$$

Portanto, $G^{0}(u) \in H^{-\alpha}(\Omega)$.

3. Para $G^{\epsilon}$ : 
Para cada $u \in H^{1}(\Omega)$ e $0<\epsilon \leq \epsilon_{0}$, vamos mostrar que $G^{\epsilon}(u) \in H^{-\alpha}(\Omega)=\left(H^{\alpha}(\Omega)\right)^{\prime}$, isto é,

$$
\begin{aligned}
G^{\epsilon}(u): H^{\alpha}(\Omega) & \rightarrow & \mathbb{R} \\
\phi & \mapsto & \left.\mapsto G^{\epsilon}(u), \phi\right\rangle,
\end{aligned}
$$

onde

$$
\left\langle G^{\epsilon}(u), \phi\right\rangle=\frac{1}{\epsilon} \int_{\omega_{\epsilon}} g(x, u(x)) \phi(x) \mathrm{d} x
$$

é uma aplicação linear contínua.

Novamente, a linearidade segue da linearidade da integral. Pelo Lema 2.7.1, temos que, dado $\phi \in H^{\alpha}(\Omega)$ e $u \in H^{1}(\Omega)$, e como $g$ é contínua, então a composta $g(\cdot, u(\cdot)) \in L^{2}\left(\omega_{\epsilon}\right)$ e $\phi \in L^{2}\left(\omega_{\epsilon}\right)$.

Usando a desigualdade de Cauchy-Schwartz, obtemos

$$
\begin{array}{r}
\left|\left\langle G^{\epsilon}(u), \phi\right\rangle\right| \leq \frac{1}{\epsilon} \int_{\omega_{\epsilon}}|g(x, u(x)) \phi(x)| \mathrm{d} x \leq\left(\frac{1}{\epsilon} \int_{\omega_{\epsilon}}|g(x, u(x))|^{2} \mathrm{~d} x\right)^{\frac{1}{2}}\left(\frac{1}{\epsilon} \int_{\omega_{\epsilon}}|\phi(x)|^{2} \mathrm{~d} x\right)^{\frac{1}{2}} \\
\leq \frac{1}{\epsilon}\|g(\cdot, u(\cdot))\|_{L^{2}\left(\omega_{\epsilon}\right)}\|\phi\|_{L^{2}\left(\omega_{\epsilon}\right)} \leq C\left(\frac{1}{\epsilon}\right)^{\frac{1}{2}}\|g(\cdot, u(\cdot))\|_{L^{2}\left(\omega_{\epsilon}\right)}\|\phi\|_{H^{\alpha}(\Omega)} .
\end{array}
$$

Portanto, $G^{\epsilon}(u) \in H^{-\alpha}(\Omega)$.

Na próxima seção, sob as condições de crescimento (H1), mostraremos que as aplicações $h^{\epsilon}$, $0 \leq \epsilon \leq \epsilon_{0}$, são localmente Lipschitzianas e o problema está bem posto.

\subsection{Existência e unicidade de soluções locais}

Nesta seção, vamos discutir a existência e unicidade de soluções para os problemas (1.1) e (1.4), ou ainda, para o problema abstrato (3.11). Para tanto, vamos usar as condições de crescimento dadas na hipótese (H1), esta abordagem foi proposta em Arrieta e Carvalho (1999) e Jiménes-Casas e Rodríguez-Bernal (2009).

Pelo Teorema 2.5.1, para garantir que existe apenas uma única solução local do problema (3.11), basta checar que as aplicações $F, G^{\epsilon}, 0 \leq \epsilon \leq \epsilon_{0}$, dadas por (3.8), (3.9) e (3.10), são localmente Lipschitziana.

Vamos enunciar, novamente, a hipótese (H1):

(H1) - Condições de crescimento crítico.

Suponha que $f, g: \bar{\Omega} \times \mathbb{R} \rightarrow \mathbb{R}$ são funções uniformemente contínuas, $f(x, \cdot), g(x, \cdot): \mathbb{R} \rightarrow \mathbb{R}$ são localmente Lipschitzianas uniformemente em $x \in \Omega$ e $x \in \bar{\Omega}$, respectivamente, e satisfazem as seguintes condições de crescimento:

i. Se $n>2$, existe $c>0$ tal que

$$
|j(x, u)-j(x, v)| \leq c|u-v|\left(|u|^{\sigma_{j}}+|v|^{\sigma_{j}}+1\right),
$$

onde $j=f$ ou $j=g$ com expoentes $\sigma_{f}$ e $\sigma_{g}$, respectivamente, tais que

$$
\sigma_{f} \leq \frac{4}{n-2} \quad \text { e } \quad \sigma_{g} \leq \frac{2}{n-2} .
$$

ii. Se $n=2$, para qualquer $\eta>0$ existe $c_{\eta}>0$ tal que

$$
|j(x, u)-j(x, v)| \leq c_{\eta}|u-v|\left(e^{\eta|u|^{2}}+e^{\eta|v|^{2}}\right),
$$

onde $j=f$ ou $j=g$. 
Para o caso $n=2$, iremos precisar do seguinte resultado que pode ser encontrado em Carvalho et al. (1997) (ver Lema 3.1.) ou como a desigualdade de Trudinger (19) em Trudinger (1967).

Lema 3.2.1 (Desigualdade de Moser-Trudinger) Se $\|u\|_{H^{1}(\mathcal{O})} \leq R$, para algum $R>0$, então existem duas constantes $\sigma, K>0$ tais que

$$
\int_{\mathcal{O}}\left(e^{\sigma|u(x)|^{2}}\right)^{2} d x \leq K|\mathcal{O}|
$$

onde $\mathcal{O}$ é um dominio limitado de $\mathbb{R}^{2}$.

Proposição 3.2.2 Suponha que $f$ satisfaz (H1). Então, a aplicação

$$
F: H^{1}(\Omega) \rightarrow H^{-1}(\Omega),
$$

dada por (3.8) é localmente Lipschitziana.

\section{Demonstração:}

Sejam $u, v \in H^{1}(\Omega) \operatorname{com}\|u\|_{H^{1}(\Omega)},\|v\|_{H^{1}(\Omega)} \leq R$, para algum $R>0$. Por definição,

$$
\|F(u)-F(v)\|_{H^{-1}(\Omega)}=\sup _{\substack{\phi \in H^{1}(\Omega) \\\|\phi\|_{H^{1}(\Omega)}=1}}|\langle F(u)-F(v), \phi\rangle| .
$$

Para cada $\phi \in H^{1}(\Omega)$, vamos estimar $|\langle F(u)-F(v), \phi\rangle|$ :

$$
|\langle F(u)-F(v), \phi\rangle| \leq \int_{\Omega}|f(x, u(x))-f(x, v(x))||\phi(x)| \mathrm{d} x .
$$

Para $n=2$ :

Dado $\eta=\sigma>0$ do Lema 3.2.1, existe $c_{\eta}>0$, tal que

$$
|\langle F(u)-F(v), \phi\rangle| \leq c_{\eta} \int_{\Omega}|u(x)-v(x)|\left(e^{\eta|u(x)|^{2}}+e^{\eta|v(x)|^{2}}\right)|\phi(x)| \mathrm{d} x .
$$

Pelo Teorema de imersão (Teorema 2.2.18), temos que $H^{1}(\Omega) \hookrightarrow L^{4}(\Omega)$. Usando as desigualdades de Cauchy-Schwartz e Minkwoski, obtemos

$$
\begin{array}{r}
|\langle F(u)-F(v), \phi\rangle| \leq c_{\eta}\|(u-v) \phi\|_{L^{2}(\Omega)}\left(\left\|e^{\eta|u|^{2}}\right\|_{L^{2}(\Omega)}+\left\|e^{\eta|v|^{2}}\right\|_{L^{2}(\Omega)}\right) \\
\leq c_{\eta}\|u-v\|_{L^{4}(\Omega)}\left(\left\|e^{\eta|u|^{2}}\right\|_{L^{2}(\Omega)}+\left\|e^{\eta|v|^{2}}\right\|_{L^{2}(\Omega)}\right)\|\phi\|_{L^{4}(\Omega)} \\
\leq \tilde{c}_{\eta}\|u-v\|_{H^{1}(\Omega)}\left(\left\|e^{\eta|u|^{2}}\right\|_{L^{2}(\Omega)}+\left\|e^{\eta|v|^{2}}\right\|_{L^{2}(\Omega)}\right)\|\phi\|_{H^{1}(\Omega)} .
\end{array}
$$

Utilizando o Lema 3.2.1, existe uma constante $K>0$ tal que

$$
|\langle F(u)-F(v), \phi\rangle| \leq c_{\eta} 2(K|\Omega|)^{\frac{1}{2}}\|u-v\|_{H^{1}(\Omega)}\|\phi\|_{H^{1}(\Omega)} .
$$

Assim, tomando o supremo com $\|\phi\|_{H^{1}(\Omega)}=1$, obtemos que existe uma constante $K_{1}=$ $K_{1}(|\Omega|, \eta, R)>0$ tal que

$$
\|F(u)-F(v)\|_{H^{-1}(\Omega)} \leq K_{1}\|u-v\|_{H^{1}(\Omega)} .
$$

Para $n>2$ :

Por (H1), 


$$
|\langle F(u)-F(v), \phi\rangle| \leq c \int_{\Omega}|u(x)-v(x)|\left(|u(x)|^{\sigma_{f}}+|v(x)|^{\sigma_{f}}+1\right)|\phi(x)| \mathrm{d} x .
$$

Utilizando a desigualdade de Hölder, com $\frac{1}{q}+\frac{1}{r}+\frac{1}{p}=1$, e a desigualdade de Minkwoski, temos

$$
|\langle F(u)-F(v), \phi\rangle| \leq c\|u-v\|_{L^{r}(\Omega)}\left(\left\|u^{\sigma_{f}}\right\|_{L^{p}(\Omega)}+\left\|v^{\sigma_{f}}\right\|_{L^{p}(\Omega)}+|\Omega|^{\frac{1}{p}}\right)\|\phi\|_{L^{q}(\Omega)} .
$$

Primeiro, note que

$$
\left\|u^{\sigma_{f}}\right\|_{L^{p}(\Omega)}=\left(\int_{\Omega}|u(x)|^{p \sigma_{f}} \mathrm{~d} x\right)^{\frac{1}{p}}=\left(\int_{\Omega}|u(x)|^{p \sigma_{f}} \mathrm{~d} x\right)^{\frac{1}{p \sigma_{f}} \sigma_{f}}=\|u\|_{L^{p \sigma_{f}}(\Omega)}^{\sigma_{\sigma}} .
$$

Assim, precisamos que

$$
H^{1}(\Omega) \hookrightarrow L^{q}(\Omega), \quad H^{1}(\Omega) \hookrightarrow L^{r}(\Omega) \quad \text { e } \quad H^{1}(\Omega) \hookrightarrow L^{p \sigma_{f}}(\Omega) .
$$

Pelo Teorema 2.2.18, devemos ter:

- $1<r \leq \frac{2 n}{n-2}$;

- $1<q \leq \frac{2 n}{n-2}$;

- $p \sigma_{f} \leq \frac{2 n}{n-2}$.

Assim,

$$
\frac{1}{r}+\frac{1}{q} \geq \frac{n-2}{n}
$$

Porém, por outro lado,

$$
\frac{1}{r}+\frac{1}{q}=1-\frac{1}{p} \geq \frac{n-2}{n}
$$

Portanto, se $H^{1}(\Omega) \hookrightarrow L^{q}(\Omega) \quad$ e $\quad H^{1}(\Omega) \hookrightarrow L^{r}(\Omega)$, segue que $p \geq \frac{n}{2}$.

Por fim, basta que $\int_{\Omega}|u(x)|^{p \sigma_{f}} \mathrm{~d} x<\infty$, mas isso decorre se tomarmos $p$ tal que $H^{1}(\Omega) \hookrightarrow$ $L^{p \sigma_{f}}(\Omega)$, isto é, pelo Teorema 2.2.18 precisamos

$$
p \sigma_{f} \leq \frac{2 n}{n-2}
$$

Porém, pela hipótese (H1) e das imersões acima,

$$
\sigma_{f} \leq \frac{4}{n-2} \quad \text { e } \quad p \geq \frac{n}{2}
$$

Logo, basta escolher, por exemplo, $p=\frac{n}{2}$ e que teremos $\int_{\Omega}|u(x)|^{p \sigma_{f}} \mathrm{~d} x<\infty$, para $v^{\sigma_{f}}$ segue da mesma forma. Portanto,

$$
|\langle F(u)-F(v), \phi\rangle| \leq c\|u-v\|_{H^{1}(\Omega)}\left(\|u\|_{H^{1}(\Omega)}^{\sigma_{f}}+\|v\|_{H^{1}(\Omega)}^{\sigma_{f}}+|\Omega|^{\frac{1}{p}}\right)\|\phi\|_{H^{1}(\Omega)} .
$$

Tomando o supremo com $\|\phi\|_{H^{1}(\Omega)}=1$, temos que existe uma constante $K_{2}=K_{2}(|\Omega|, R)>0$ tal que

$$
\|F(u)-F(v)\|_{H^{-1}(\Omega)} \leq K_{2}\|u-v\|_{H^{1}(\Omega)} .
$$


Proposição 3.2.3 Suponha que g satisfaz (H1). Então, para $\epsilon \in\left[0, \epsilon_{0}\right]$, a aplicação

$$
G^{\epsilon}: H^{1}(\Omega) \rightarrow H^{-1}(\Omega)
$$

dada por (3.9) e (3.10) é localmente Lipschitziana, uniformemente em $\epsilon$.

\section{Demonstração:}

Sejam $u, v \in H^{1}(\Omega) \operatorname{com}\|u\|_{H^{1}(\Omega)},\|v\|_{H^{1}(\Omega)} \leq R$, para algum $R>0$.

Por definição, para cada $\epsilon \in\left[0, \epsilon_{0}\right]$,

$$
\left\|G^{\epsilon}(u)-G^{\epsilon}(v)\right\|_{H^{-1}(\Omega)}=\sup _{\substack{\phi \in H^{1}(\Omega) \\\|\phi\|_{H^{1}(\Omega)}=1}}\left|\left\langle G^{\epsilon}(u)-G^{\epsilon}(v), \phi\right\rangle\right| .
$$

Para cada $\phi \in H^{1}(\Omega)$ e $0<\epsilon \leq \epsilon_{0}$, vamos estimar $\left|\left\langle G^{\epsilon}(u)-G^{\epsilon}(v), \phi\right\rangle\right|$ :

$$
\left|\left\langle G^{\epsilon}(u)-G^{\epsilon}(v), \phi\right\rangle\right| \leq \frac{1}{\epsilon} \int_{\omega_{\epsilon}}|g(x, u(x))-g(x, v(x))||\phi(x)| \mathrm{d} x
$$

Para $n=2$ :

Dado $\eta=\sigma>0$ dado em Lema 3.2.1, pela hipótese (H1) existe $c_{\eta}>0$, tal que

$$
\left|\left\langle G^{\epsilon}(u)-G^{\epsilon}(v), \phi\right\rangle\right| \leq c_{\eta} \frac{1}{\epsilon} \int_{\omega_{\epsilon}}|u(x)-v(x)|\left(e^{\eta|u(x)|^{2}}+e^{\eta|v(x)|^{2}}\right)|\phi(x)| \mathrm{d} x .
$$

Note que podemos utilizar o Lema 2.7.1 com $1<q<\infty$ quando $H_{p}^{s}(\Omega)=H^{1}(\Omega)$ e $n=2$, pois $\frac{1}{2}<1$ e $1-\frac{n}{2} \geq-\frac{n-1}{q} \Rightarrow 0 \geq-\frac{1}{q}$. Vamos usar $q=4$.

Dessa forma, utilizando as desigualdades de Cauchy-Schwartz e Minkwoski e o Lema 2.7.1, obtemos

$$
\begin{aligned}
&\left|\left\langle G^{\epsilon}(u)-G^{\epsilon}(v), \phi\right\rangle\right| \leq c_{\eta}\left(\frac{1}{\epsilon} \int_{\omega_{\epsilon}}|u(x)-v(x)|^{4} \mathrm{~d} x\right)^{\frac{1}{4}}\left(\frac{1}{\epsilon} \int_{\omega_{\epsilon}}|\phi(x)|^{4} \mathrm{~d} x\right)^{\frac{1}{4}} \\
& {\left[\left(\frac{1}{\epsilon} \int_{\omega_{\epsilon}}\left|e^{\eta|u(x)|^{2}}\right|^{2} \mathrm{~d} x\right)^{\frac{1}{2}}+\left(\frac{1}{\epsilon} \int_{\omega_{\epsilon}}\left|e^{\eta|v(x)|^{2}}\right|^{2} \mathrm{~d} x\right)^{\frac{1}{2}}\right] } \\
& \leq c_{\eta} c^{*}\|u-v\|_{H^{1}(\Omega)}\|\phi\|_{H^{1}(\Omega)}\left[\left(\frac{1}{\epsilon} \int_{\omega_{\epsilon}}\left|e^{\eta|u(x)|^{2}}\right|^{2} \mathrm{~d} x\right)^{\frac{1}{2}}+\left(\frac{1}{\epsilon} \int_{\omega_{\epsilon}}\left|e^{\eta|v(x)|^{2}}\right|^{2} \mathrm{~d} x\right)^{\frac{1}{2}}\right] .
\end{aligned}
$$

Utilizando o Lema 3.2.1, existe $K>0$ tal que

$$
\left|\left\langle G^{\epsilon}(u)-G^{\epsilon}(v), \phi\right\rangle\right| \leq c_{\eta} C\left(\frac{K\left|\omega_{\epsilon}\right|}{\epsilon}\right)^{\frac{1}{2}}\|u-v\|_{H^{1}(\Omega)}\|\phi\|_{H^{1}(\Omega)} .
$$

Utilizando que a medida de $\omega_{\epsilon}$ é da ordem de $\epsilon \operatorname{com}\left|\omega_{\epsilon}\right| \leq k \epsilon|\partial \Omega|$, para algum $k>0$ independente de $\epsilon$, então

$$
\left|\left\langle G^{\epsilon}(u)-G^{\epsilon}(v), \phi\right\rangle\right| \leq 2 c_{\eta} C(K k|\partial \Omega|)^{\frac{1}{2}}\|u-v\|_{H^{1}(\Omega)}\|\phi\|_{H^{1}(\Omega)} .
$$

Tomando o supremo com $\|\phi\|_{H^{1}(\Omega)}=1$, temos que existe uma constate $K_{3}=K_{3}(\eta,|\partial \Omega|, R)>0$ independente de $\epsilon$ tal que

$$
\left\|G^{\epsilon}(u)-G^{\epsilon}(v)\right\|_{H^{1}(\Omega)} \leq K_{3}\|u-v\|_{H^{1}(\Omega)}
$$


Para $n>2$ :

Por (H1),

$$
\left|\left\langle G^{\epsilon}(u)-G^{\epsilon}(v), \phi\right\rangle\right| \leq c \frac{1}{\epsilon} \int_{\omega_{\epsilon}}|u(x)-v(x)|\left(|u(x)|^{\sigma_{g}}+|v(x)|^{\sigma_{g}}+1\right)|\phi(x)| \mathrm{d} x .
$$

Utilizando a desigualdade de Hölder, com $\frac{1}{q}+\frac{1}{r}+\frac{1}{p}=1$, temos

$$
\begin{array}{r}
\left|\left\langle G^{\epsilon}(u)-G^{\epsilon}(v), \phi\right\rangle\right| \leq c\left(\frac{1}{\epsilon} \int_{\omega_{\epsilon}}|u(x)-v(x)|^{r} \mathrm{~d} x\right)^{\frac{1}{r}}\left(\frac{1}{\epsilon} \int_{\omega_{\epsilon}}|\phi(x)|^{q} \mathrm{~d} x\right)^{\frac{1}{q}} \\
\left(\frac{1}{\epsilon} \int_{\omega_{\epsilon}}\left(|u(x)|^{\sigma_{g}}+|v(x)|^{\sigma_{g}}+1\right)^{p} \mathrm{~d} x\right)^{\frac{1}{p}} .
\end{array}
$$

Usando também a desigualdade de Minkwoski, obtemos

$$
\begin{array}{r}
\left|\left\langle G^{\epsilon}(u)-G^{\epsilon}(v), \phi\right\rangle\right| \leq c\left(\frac{1}{\epsilon}\right)^{\frac{1}{r}}\|u-v\|_{L^{r}\left(\omega_{\epsilon}\right)}\left(\frac{1}{\epsilon}\right)^{\frac{1}{q}}\|\phi\|_{L^{q}\left(\omega_{\epsilon}\right)} \\
{\left[\left(\frac{1}{\epsilon}\right)^{\frac{1}{p}}\left\|u^{\sigma_{g}}\right\|_{L^{p}\left(\omega_{\epsilon}\right)}+\left(\frac{1}{\epsilon}\right)^{\frac{1}{p}}\left\|v^{\sigma_{g}}\right\|_{L^{p}\left(\omega_{\epsilon}\right)}+\left(\frac{\left|\omega_{\epsilon}\right|}{\epsilon}\right)^{\frac{1}{p}}\right] .}
\end{array}
$$

Primeiro, note que $\left(\frac{1}{\epsilon}\right)^{\frac{1}{p}}\left\|u^{\sigma_{g}}\right\|_{L^{p}\left(\omega_{\epsilon}\right)}=\left[\left(\frac{1}{\epsilon}\right)^{\frac{1}{p \sigma_{g}}}\|u\|_{L^{p \sigma_{g}\left(\omega_{\epsilon}\right)}}\right]^{\sigma_{g}}$, análogo para $v^{\sigma_{g}}$.

Para aplicarmos o Lema 2.7.1 com $u \in H_{p}^{s}(\Omega)=H^{1}(\Omega)$, vamos precisar que

$$
1-\frac{n}{2} \geq-\frac{n-1}{q}, \quad 1-\frac{n}{2} \geq-\frac{n-1}{r} \quad \text { e } \quad 1-\frac{n}{2} \geq-\frac{n-1}{\sigma_{g} p} .
$$
que

Portanto, basta escolher $1<r, q \leq \frac{2(n-1)}{n-2}$. Somando o inverso das duas parcelas, obtemos

$$
\frac{1}{r}+\frac{1}{q} \geq \frac{n-2}{n-1} .
$$

Por outro lado, $\frac{1}{r}+\frac{1}{q}=1-\frac{1}{p}, \operatorname{logo}$

$$
\frac{1}{p} \leq \frac{1}{n-1} \quad \text { ou } \quad p \geq n-1
$$

Assim, se $1<r, q \leq \frac{2(n-1)}{n-2}$. concluímos que $p \geq n-1$ e vale o Lema 2.7.1, isto é, existe $c>0$ independente de $\epsilon$ tal que

$$
\left(\frac{1}{\epsilon}\right)^{\frac{1}{r}}\|u-v\|_{L^{r}\left(\omega_{\epsilon}\right)} \leq C\|u-v\|_{H^{1}(\Omega)} \quad \text { e } \quad\left(\frac{1}{\epsilon}\right)^{\frac{1}{q}}\|\phi\|_{L^{q}\left(\omega_{\epsilon}\right)} \leq C\|\phi\|_{H^{1}(\Omega)} .
$$

Por fim, também precisamos que $p \sigma_{g} \leq \frac{2(n-1)}{n-2}$. Porém pela hipótese (H1) e pela imersão acima,

$$
\sigma_{g} \leq \frac{2}{n-2} \quad \text { e } \quad p \geq n-1
$$


Portanto, basta escolher, por exemplo, $p=n-1$ que teremos

$$
\left(\frac{1}{\epsilon}\right)^{\frac{1}{p}}\left\|u^{\sigma_{g}}\right\|_{L^{p}\left(\omega_{\epsilon}\right)} \leq C\|u\|_{H^{1}(\Omega)}^{\sigma_{g}},
$$

analogamente para $v^{\sigma_{g}}$. Assim, utilizando que $\left|\omega_{\epsilon}\right| \leq k \epsilon|\partial \Omega|$, para algum $k>0$ independente de $\epsilon$, obtemos

$$
\left|\left\langle G^{\epsilon}(u)-G^{\epsilon}(v), \phi\right\rangle\right| \leq \tilde{c}\|u-v\|_{H^{1}(\Omega)}\left(\|u\|_{H^{1}(\Omega)}^{\sigma_{g}}+\|v\|_{H^{1}(\Omega)}^{\sigma_{g}}+1\right)\|\phi\|_{H^{1}(\Omega)},
$$

onde $\tilde{c}=\tilde{c}(|\partial \Omega|)>0$ independe de $\epsilon$.

Tomando o supremo com $\|\phi\|_{H^{1}(\Omega)}=1$, temos que existe $K_{4}=K_{4}(|\partial \Omega|, R)>0$ independente de $\epsilon$ tal que

$$
\left\|G^{\epsilon}(u)-G^{\epsilon}(v)\right\|_{H^{-1}(\Omega)} \leq K_{4}\|u-v\|_{H^{1}(\Omega)} .
$$

Até então mostramos que para cada $0<\epsilon \leq \epsilon_{0}, G^{\epsilon}$ é uma aplicação localmente Lipschitziana, uniformemente em $\epsilon$.

Agora, para cada $\phi \in H^{1}(\Omega)$, vamos estimar $\left|\left\langle G^{0}(u)-G^{0}(v), \phi\right\rangle\right|$ :

$$
\begin{aligned}
\left|\left\langle G^{0}(u)-G^{0}(v), \phi\right\rangle\right| \leq & \int_{\partial \Omega}|[\gamma(g(x, u(x)))-\gamma(g(x, v(x)))] \gamma(\phi(x))| \mathrm{d} S \\
& =\int_{\partial \Omega}|[\gamma(g(x, u(x))-g(x, v(x)))] \gamma(\phi(x))| \mathrm{d} S .
\end{aligned}
$$

Para $n=2$ :

Dado $\eta=\sigma>0$ do Lema 3.2.1, pela hipótese (H1), existe $c_{\eta}>0$, tal que

$$
\left|\left\langle G^{0}(u)-G^{0}(v), \phi\right\rangle\right| \leq c_{\eta} \int_{\partial \Omega}|\gamma(u(x)-v(x))| \gamma\left(e^{\eta|u(x)|^{2}}+e^{\eta|v(x)|^{2}}\right)|\gamma(\phi(x))| \mathrm{d} S .
$$

Utilizando as desigualdades de Cauchy-Schwartz e Minkwoski, temos

$$
\left|\left\langle G^{0}(u)-G^{0}(v), \phi\right\rangle\right| \leq c_{\eta}\|\gamma(u-v)\|_{L^{4}(\partial \Omega)}\left[\left(\int_{\partial \Omega}\left|\gamma\left(e^{\eta|u|^{2}}\right)\right|^{2} \mathrm{~d} S\right)^{\frac{1}{2}}+\left(\int_{\partial \Omega}\left|\gamma\left(e^{\eta|v|^{2}}\right)\right|^{2} \mathrm{~d} S\right)^{\frac{1}{2}}\right]\|\gamma(\phi)\|_{L^{4}(\partial \Omega)} .
$$

Pelo Lema 2.2.19, o operador traço $\gamma: H^{1}(\Omega) \rightarrow L^{4}(\partial \Omega)$ é linear contínuo.

Utilizando novamente a desigualdade do Lema 3.2.1 e substituindo,

$$
\left|\left\langle G^{0}(u)-G^{0}(v), \phi\right\rangle\right| \leq \tilde{c}_{\eta}\|u-v\|_{H^{1}(\Omega)} 2(K|\partial \Omega|)^{\frac{1}{2}}\|\phi\|_{H^{1}(\Omega)} .
$$

Sob essas condições, tomando o supremo com $\|\phi\|_{H^{1}(\Omega)}=1$, segue que existe uma constante $K_{5}=K_{5}(|\partial \Omega|, \eta, R)>0$ tal que

$$
\left\|G^{0}(u)-G^{0}(v)\right\|_{H^{-1}(\Omega)} \leq K_{5}\|u-v\|_{H^{1}(\Omega)} .
$$

Para $n>2$ :

Por (H1),

$$
\left|\left\langle G^{0}(u)-G^{0}(v), \phi\right\rangle\right| \leq c \int_{\partial \Omega}|\gamma(u(x)-v(x))| \gamma\left(|u(x)|^{\sigma_{g}}+|v(x)|^{\sigma_{g}}+1\right)|\gamma(\phi(x))| \mathrm{d} S .
$$

Utilizando a desigualdade de Hölder, com $\frac{1}{q}+\frac{1}{r}+\frac{1}{p}=1$, e a desigualdade de Minkwoski, obtemos $\left|\left\langle G^{0}(u)-G^{0}(v), \phi\right\rangle\right| \leq c\|\gamma(u-v)\|_{L^{r}(\partial \Omega)}\left[\|\gamma(u)\|_{L^{\sigma_{g} p}(\partial \Omega)}^{\sigma_{g}}+\|\gamma(v)\|_{L^{\sigma_{g} p}(\partial \Omega)}^{\sigma_{g}}+|\partial \Omega|^{\frac{1}{p}}\right]\|\gamma(\phi)\|_{L^{q}(\partial \Omega)}$. 
Neste caso também precisamos que o operador traço seja contínuo se definido em

$$
H^{1}(\Omega) \rightarrow L^{q}(\partial \Omega), \quad H^{1}(\Omega) \rightarrow L^{r}(\partial \Omega) \quad \text { e } \quad H^{1}(\Omega) \rightarrow L^{p \sigma_{g}}(\partial \Omega) .
$$

Então, pelo Lema 2.2.19, é suficiente que:

$$
1-\frac{n}{2} \geq-\frac{n-1}{q}, \quad 1-\frac{n}{2} \geq-\frac{n-1}{r} \quad \text { e } \quad 1-\frac{n}{2} \geq-\frac{n-1}{p \sigma_{g}} .
$$

Escolhendo $1<r, q \leq \frac{2(n-1)}{n-2}$. Somando o inverso das duas parcelas, obtemos que

$$
\frac{1}{r}+\frac{1}{q} \geq \frac{n-2}{n-1}
$$

Por outro lado, $\frac{1}{r}+\frac{1}{q}=1-\frac{1}{p}, \operatorname{logo}$

$$
\frac{1}{p} \leq \frac{1}{n-1} \quad \text { ou } \quad p \geq n-1
$$

Assim, se $1<r, q \leq \frac{2(n-1)}{n-2}$. concluímos que $p \geq n-1$ e que o traço é contínuo, isto é,

$$
\|\gamma(u-v)\|_{L^{r}(\partial \Omega)} \leq c\|u-v\|_{H^{1}(\Omega)} \quad \text { e } \quad\|\gamma(\phi)\|_{L^{q}(\partial \Omega)} \leq c\|\phi\|_{H^{1}(\Omega)} .
$$

Por fim, precisamos analisar $\|\gamma(u)\|_{L^{\sigma_{g} p}(\partial \Omega)}$.

Pelo Lema 2.2.19 sabemos que

$$
\|\gamma(u)\|_{L^{p \sigma_{g}(\partial \Omega)}} \leq k\|u\|_{H^{1}(\Omega)},
$$

desde que

$$
1-\frac{n}{2} \geq-\frac{n-1}{p \sigma_{g}} \Rightarrow p \sigma_{g} \leq \frac{2(n-1)}{n-2}
$$

Por (H1), sabemos que

$$
\sigma_{g} \leq \frac{2}{n-2}
$$

portanto, basta escolher, por exemplo, $p=n-1$, logo

$$
\left|\left\langle G^{0}(u)-G^{0}(v), \phi\right\rangle\right| \leq \tilde{c}\|u-v\|_{H^{1}(\Omega)}\left(\|u\|_{H^{1}(\Omega)}^{\sigma_{g}}+\|v\|_{H^{1}(\Omega)}^{\sigma_{g}}+1\right)\|\phi\|_{H^{1}(\Omega)},
$$

onde $\tilde{c}=\tilde{c}(|\partial \Omega|)>0$.

Tomando o supremo com $\|\phi\|_{H^{1}(\Omega)}=1$, temos que existe uma constante $K_{6}=K_{6}(|\partial \Omega|, R)>0$ tal que

$$
\left\|G^{0}(u)-G^{0}(v)\right\|_{H^{-1}(\Omega)} \leq K_{6}\|u-v\|_{H^{1}(\Omega)} .
$$

Como estamos estudando um problema no qual as não-linearidades podem ter a mesma ordem do operador, enunciamos o teorema a seguir, no qual garante a existência local de soluções fracas dos problemas (1.1) e (1.4), ou ainda (3.11), e sua prova é uma consequência imediata dos resultados discutidos em Arrieta e Carvalho (1999).

Teorema 3.2.4 [Existência, unicidade e dependência contínua]

Suponha que as hipóteses (H1) e (P) sejam satisfeitas. Então, para cada $0 \leq \epsilon \leq \epsilon_{0}$ e $u_{0} \in$ $H^{1}(\Omega)$, existem $T^{\epsilon}=T^{\epsilon}\left(u_{0}\right)>0$ e uma única função contínua $u^{\epsilon}:\left[0, T^{\epsilon}\right) \rightarrow H^{1}(\Omega)$ solução de 
(3.11), ou ainda $u^{\epsilon}$ solução de (1.1) e $u^{0}$ de solução de (1.4), tal que

$$
u^{\epsilon}(t)=e^{-A^{\epsilon} t} u_{0}+\int_{0}^{t} e^{-A^{\epsilon}(t-s)} h^{\epsilon}\left(u^{\epsilon}(s)\right) d s, \quad t \in\left[0, T^{\epsilon}\right),
$$

onde $\left[0, T^{\epsilon}\right)$ é o intervalo maximal de existência da solução. E ainda, essa solução depende continuamente do dado inicial.

Observação 3.2.5 A existência e unicidade de equações diferenciais estão condicionadas, no geral, a verificar que as funçôes envolvidas nas equação satisfazem a condiçâo de Lipschtz, contudo, nem sempre é possível concluir essa demostração para qualquer espaço. Discutiremos no Apêndice A outra forma de obter essa condição de Lipschitz para as não linearidades abstratas.

\subsection{Existência global e limitação uniforme de soluções}

Nesta seção vamos concluir que as soluções de (1.1) e (1.4), ou ainda de (3.11) são globalmente definidas e uniformemente limitadas em $H^{1}(\Omega)$ e $L^{\infty}(\Omega)$ para todo tempo. Vamos utilizar resultados de comparação para comparar as soluções de (1.1) e (1.4) com as soluções de problemas parabólicos lineares com termos concentrados. A vantagem dessa comparação é que o comportamento de problemas parabólicos lineares já foi analisado em Rodríguez-Bernal (2011) e Jiménes-Casas e Rodríguez-Bernal (2011). Para tanto, além da hipótese (H1), vamos precisar da condição de sinal (H2) para mostrarmos que as soluções de (1.1) e (1.4) são uniformemente limitadas em intervalos compactos de $\mathbb{R}^{+}$e consequentemente concluir que são globalmente definidas. Agora, para obtermos a limitação uniforme das soluções de (1.1) e (1.4), para todo tempo, as hipóteses (H1) e (H2) não serão suficientes, precisamos também da condição de dissipatividade (H3). Novamente, vamos enunciar aqui as hipóteses (H2) e (H3).

\section{(H2) - Condição de sinal.}

Suponha que existem funções $C \in L^{p}(\Omega), 0 \leq D \in L^{p}(\Omega)$, onde $p>\frac{n}{2}, E \in L^{q}(\Omega), 0 \leq F \in$ $L^{q}(\Omega)$, onde $q>n-1$, tais que

$$
\begin{array}{llll}
s f(x, s) \leq C(x) s^{2}+D(x)|s|, & x \in \Omega \quad \text { e } & s \in \mathbb{R}, \\
s g(x, s) \leq E(x) s^{2}+F(x)|s|, & x \in \bar{\Omega} \quad \text { e } \quad & s \in \mathbb{R} .
\end{array}
$$

Além disso, para $\epsilon \in\left(0, \epsilon_{0}\right]$, existem constantes $K_{2}, K_{3}>0$ independentes de $\epsilon$ tais que

$$
\begin{gathered}
\frac{1}{\epsilon} \int_{\omega_{\epsilon}}|E|^{r_{1}} \mathrm{~d} x \leq K_{2}, \quad \text { onde } \quad r_{1}>n-1 ; \\
\frac{1}{\epsilon} \int_{\omega_{\epsilon}}|F|^{r_{2}} \mathrm{~d} x \leq K_{3}, \quad \text { onde } \quad r_{2}>\max \left\{1, \frac{2(n-1)}{n}\right\} .
\end{gathered}
$$

(H3) - Condição de dissipação.

Suponha que o primeiro autovalor, $\lambda_{1}$, do problema a seguir é positivo

$$
\begin{cases}-\Delta \phi(x)-C(x) \phi(x)+\mu \phi(x)=\lambda_{1} \phi(x), & x \in \Omega \\ \frac{\partial \phi}{\partial \vec{n}}(x)+V_{0}(x) \phi(x)=E(x) \phi(x), & x \in \partial \Omega\end{cases}
$$

onde $C$ e $E$ são como em (H2).

Usaremos a (H2) para comparar as soluções de (1.1) e (1.4) com as soluções dos seguintes problemas parabólicos lineares: 


$$
\begin{cases}\frac{\partial w^{\epsilon}}{\partial t}+L^{\epsilon} w^{\epsilon}=C(x) w^{\epsilon}+D(x)+\frac{1}{\epsilon} \chi_{\omega_{\epsilon}}(x)\left[E(x) w^{\epsilon}+F(x)\right], & (t, x) \in(0, \infty) \times \Omega \\ \frac{\partial w^{\epsilon}}{\partial \vec{n}}=0, & (t, x) \in(0, \infty) \times \partial \Omega \\ w^{\epsilon}(0)=\left|u_{0}\right|, & \bar{\Omega}\end{cases}
$$

onde $L^{\epsilon}$ é dado por (1.2), e

$$
\begin{cases}\frac{\partial w^{0}}{\partial t}+L^{0} w^{0}=C(x) w^{0}+D(x), & (t, x) \in(0, \infty) \times \Omega, \\ \frac{\partial w^{0}}{\partial \vec{n}}+V_{0}(x) w^{0}=E(x) w^{0}+F(x), & (t, x) \in(0, \infty) \times \partial \Omega \\ w^{0}(0)=\left|u_{0}\right|, & \bar{\Omega}\end{cases}
$$

onde $L^{0}$ é dado por (1.5).

De forma análoga aos problemas (1.1) e (1.4), vamos escrever as formas abstratas de (3.12) e (3.13) assoaciadas, respectivamente, às suas fórmulas variacionais:

$$
\begin{array}{r}
\int_{\Omega}\left[\frac{\partial w^{\epsilon}}{\partial t}(t, x) \phi(x)+\mu w^{\epsilon}(t, x) \phi(x)+\nabla w^{\epsilon}(t, x) \nabla \phi(x)\right] \mathrm{d} x+\frac{1}{\epsilon} \int_{\omega_{\epsilon}} V_{\epsilon}(x) w^{\epsilon}(t, x) \phi(x) \mathrm{d} x= \\
\int_{\Omega}\left[C(x) w^{\epsilon}(t, x)+D(x)\right] \phi(x) \mathrm{d} x+\frac{1}{\epsilon} \int_{\omega_{\epsilon}}\left[E(x) w^{\epsilon}(t, x)+F(x)\right] \phi(x) \mathrm{d} x, \\
\int_{\Omega}\left[\frac{\partial w^{0}}{\partial t}(t, x) \phi(x)+\mu w^{0}(t, x) \phi(x)+\nabla w^{0}(t, x) \nabla \phi(x)\right] \mathrm{d} x+\int_{\partial \Omega} V_{0}(x) w^{0}(t, x) \phi(x) \mathrm{d} S= \\
\int_{\Omega}\left[C(x) w^{0}(t, x)+D(x)\right] \phi(x) \mathrm{d} x+\int_{\partial \Omega}\left[E(x) w^{0}(t, x)+F(x)\right] \phi(x) \mathrm{d} S .
\end{array}
$$

Definimos $H^{0}: H^{1}(\Omega) \rightarrow H^{-\alpha}(\Omega), \frac{1}{2}<\alpha \leq 1$, por

$$
\left\langle H^{0}(w), \phi\right\rangle=\int_{\Omega}[C(x) w(x)+D(x)] \phi(x) \mathrm{d} x+\int_{\partial \Omega} \gamma(E(x) w(x)+F(x)) \gamma(\phi(x)) \mathrm{d} S,
$$

para $w \in H^{1}(\Omega)$ e $\phi \in H^{\alpha}(\Omega)$.

Para cada $0<\epsilon \leq \epsilon_{0}$, definimos $H^{\epsilon}: H^{1}(\Omega) \rightarrow H^{-\alpha}(\Omega), \frac{1}{2}<\alpha \leq 1$, por

$$
\left\langle H^{\epsilon}(w), \phi\right\rangle=\int_{\Omega}[C(x) w(x)+D(x)] \phi(x) \mathrm{d} x+\frac{1}{\epsilon} \int_{\omega_{\epsilon}}[E(x) w(x)+F(x)] \phi(x) \mathrm{d} x,
$$

para $w \in H^{1}(\Omega)$ e $\phi \in H^{\alpha}(\Omega)$.

Procedendo de maneira análoga a Seção 3.1, a fórmula abstrata dos problemas (3.12) e (3.13) é dada por

$$
\left\{\begin{array}{l}
w_{t}^{\epsilon}+A^{\epsilon} w^{\epsilon}=H^{\epsilon}\left(w^{\epsilon}\right), \quad t>0 \\
w^{\epsilon}(0)=\left|u_{0}\right| \in H^{1}(\Omega)
\end{array}\right.
$$

com $0 \leq \epsilon \leq \epsilon_{0}$.

A existência global e unicidade de soluções fracas dos problemas parabólicos lineares (3.12) e (3.13), ou ainda (3.14), em $H^{1}(\Omega)$ seguem dos resultados de Rodríguez-Bernal (2011) (Corolário 2.6) ou Jiménes-Casas e Rodríguez-Bernal (2011) (Teorema 3.2). 
Agora, para garantirmos existência global de soluções de (1.1) e (1.4), vamos usar o Corolário 2.5.3, mas para tanto, primeiramente, precisamos provar que as aplicações $h^{\epsilon}: H^{1}(\Omega) \rightarrow H^{-\alpha}(\Omega)$, $\operatorname{com} \frac{1}{2}<\alpha \leq 1$ são uniformemente limitadas sob limitados, onde $h^{\epsilon}=F+G^{\epsilon}, \epsilon \in\left[0, \epsilon_{0}\right]$, com $F$ e $G^{\epsilon}$ dadas em (3.8), (3.10) e (3.9).

Lema 3.3.1 Suponha que $f, g: \bar{\Omega} \times \mathbb{R} \rightarrow \mathbb{R}$ são funçôes uniformemente contínuas. Se $u \in H^{1}(\Omega) \cap$ $L^{\infty}(\Omega)$ e $\|u\|_{L^{\infty}(\Omega)} \leq R$, para alguma constante $R>0$, e $\frac{1}{2}<\alpha \leq 1$, então existe uma constante $C=C(R)>0$ independente de $\epsilon$ tal que

$$
\left\|h^{\epsilon}(u)\right\|_{H^{-\alpha}(\Omega)} \leq C, \quad 0 \leq \epsilon \leq \epsilon_{0} .
$$

\section{Demonstração:}

Como $h^{\epsilon}=F+G^{\epsilon}, \epsilon \in\left(0, \epsilon_{0}\right]$, e $h^{0}=F+G^{0}$, vamos mostrar que $F$ e $G^{\epsilon}, 0 \leq \epsilon \leq \epsilon_{0}$, onde $F, G^{\epsilon}$ são dadas por (3.8), (3.9) e (3.10), são uniformemente limitadas sob limitados de $L^{\infty}(\Omega)$.

Primeiro para $F$. Por definição,

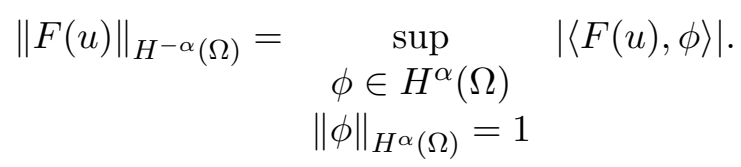

Para cada $\phi \in H^{\alpha}(\Omega)$,

$$
|\langle F(u), \phi\rangle| \leq \int_{\Omega}|f(x, u(x)) \phi(x)| \mathrm{d} x .
$$

Como $f$ é uniformemente contínua em $\bar{\Omega} \times \mathbb{R}$ e $\|u\|_{L^{\infty}(\Omega)} \leq R$, então existe uma constante $L_{1}=L_{1}(R)>0$ tal que $\|f(\cdot, u(\cdot))\|_{L^{\infty}(\Omega)} \leq L_{1}$. Logo,

$$
|\langle F(u), \phi\rangle| \leq\|f(\cdot, u(\cdot))\|_{L^{\infty}(\Omega)} \int_{\Omega}|\phi(x)| \mathrm{d} x \leq L_{1} \int_{\Omega}|\phi(x)| \mathrm{d} x .
$$

Utilizando a desigualdade de Cauchy-Schwartz,

$$
|\langle F(u), \phi\rangle| \leq|\Omega|^{\frac{1}{2}} L_{1}\|\phi\|_{L^{2}(\Omega)} .
$$

Pelo Teorema 2.2.18, $H^{\alpha}(\Omega) \hookrightarrow L^{2}(\Omega)$, com $\frac{1}{2}<\alpha \leq 1$. Logo,

$$
|\langle F(u), \phi\rangle| \leq|\Omega|^{\frac{1}{2}} \tilde{L}_{1}\|\phi\|_{H^{\alpha}(\Omega)} .
$$

Tomando o supremo com $\|\phi\|_{H^{\alpha}(\Omega)}=1$, obtemos

$$
\|F(u)\|_{H^{-\alpha}(\Omega)} \leq C_{1},
$$

onde $C_{1}$ depende apenas de $R$, uma vez que $\Omega$ é limitado.

Considere $0 \leq \epsilon \leq \epsilon_{0}$. Por definição,

$$
\left\|G^{\epsilon}(u)\right\|_{H^{-\alpha}(\Omega)}=\sup _{\substack{\phi \in H^{\alpha}(\Omega) \\\|\phi\|_{H^{\alpha}(\Omega)}=1}}\left|\left\langle G^{\epsilon}(u), \phi\right\rangle\right| .
$$

Para cada $\phi \in H^{\alpha}(\Omega)$ e $0<\epsilon \leq \epsilon_{0}$, vamos estimar $\left|\left\langle G^{\epsilon}(u), \phi\right\rangle\right|$ :

$$
\left|\left\langle G^{\epsilon}(u), \phi\right\rangle\right| \leq \frac{1}{\epsilon} \int_{\omega_{\epsilon}}|g(x, u(x)) \phi(x)| \mathrm{d} x .
$$

Como $g$ é uniformemente contínua em $\bar{\Omega} \times \mathbb{R}$ e $\|u\|_{L^{\infty}(\Omega)} \leq R$, então existe uma constante $L_{2}=L_{2}(R)>0$ tal que $\|g(\cdot, u(\cdot))\|_{L^{\infty}(\bar{\Omega})} \leq L_{2}$. Logo, 


$$
\begin{array}{r}
\left|\left\langle G^{\epsilon}(u), \phi\right\rangle\right| \leq\|g(\cdot, u(\cdot))\|_{L^{\infty}\left(\omega_{\epsilon}\right)} \frac{1}{\epsilon} \int_{\omega_{\epsilon}}|\phi(x)| \mathrm{d} x \leq\|g(\cdot, u(\cdot))\|_{L^{\infty}(\bar{\Omega})} \frac{1}{\epsilon} \int_{\omega_{\epsilon}}|\phi(x)| \mathrm{d} x \\
\leq L_{2} \frac{1}{\epsilon} \int_{\omega_{\epsilon}}|\phi(x)| \mathrm{d} x .
\end{array}
$$

Utilizando a desigualdade de Cauchy-Schwartz e $\left|\omega_{\epsilon}\right| \leq k \epsilon|\partial \Omega|$, para algum $k>0$ independente de $\epsilon$, obtemos

$$
\left|\left\langle G^{\epsilon}(u), \phi\right\rangle\right| \leq L_{2}(k|\partial \Omega|)^{\frac{1}{2}}\left(\frac{1}{\epsilon} \int_{\omega_{\epsilon}}|\phi(x)|^{2} \mathrm{~d} x\right)^{\frac{1}{2}} .
$$

Utilizando o Lema 2.7.1, existe $C>0$ independente de $\epsilon$ tal que

$$
\left|\left\langle G^{\epsilon}(u), \phi\right\rangle\right| \leq L_{2}(k|\partial \Omega|)^{\frac{1}{2}} C\|\phi\|_{H^{\alpha}(\Omega)} .
$$

Agora, tomando o supremo com $\|\phi\|_{H^{\alpha}(\Omega)}=1$, obtemos

$$
\left\|G^{\epsilon}(u)\right\|_{H^{-\alpha}(\Omega)} \leq C_{2},
$$

onde $C_{2}=C_{2}(R)>0$ independe de $\epsilon$.

Por fim, para $\epsilon=0$ e $\phi \in H^{\alpha}(\Omega)$, temos

$$
\left|\left\langle G^{0}(u), \phi\right\rangle\right| \leq \int_{\partial \Omega}|\gamma(g(x, u(x))) \gamma(\phi(x))| \mathrm{d} S .
$$

Como $g$ é uniformemente contínua em $\bar{\Omega} \times \mathbb{R}$ e $\|u\|_{L^{\infty}(\Omega)} \leq R$, então existe uma constante $L_{3}=L_{3}(R)>0$ tal que $\|\gamma(g(\cdot, u(\cdot)))\|_{L^{\infty}(\partial \Omega)} \leq L_{3}$. Assim, utilizando a desigualdade de CauchySchwartz e o Lema 2.2.19 para garantir que o traço é um operador contínuo de $H^{\alpha}(\Omega)$ em $L^{2}(\partial \Omega)$, obtemos

$$
\left|\left\langle G^{0}(u), \phi\right\rangle\right| \leq\|\gamma(g(\cdot, u(\cdot)))\|_{L^{\infty}(\partial \Omega)}(|\partial \Omega|)^{\frac{1}{2}}\|\gamma(\phi)\|_{L^{2}(\partial \Omega)} \leq \tilde{L}_{3}(|\partial \Omega|)^{\frac{1}{2}}\|\phi\|_{H^{\alpha}(\Omega)} .
$$

Tomando o supremo com $\|\phi\|_{H^{\alpha}(\Omega)}=1$, temos

$$
\left\|G^{0}(u)\right\|_{H^{-\alpha}(\Omega)} \leq C_{3},
$$

onde $C_{3}=C_{3}(R)>0$ independe de $\epsilon$.

Lema 3.3.2 Suponha que $f, g: \bar{\Omega} \times \mathbb{R} \rightarrow \mathbb{R}$ são funções uniformemente contínuas, $f(x, \cdot), g(x, \cdot)$ : $\mathbb{R} \rightarrow \mathbb{R}$ são localmente Lipschitzianas uniformemente em $x \in \Omega$ e $x \in \bar{\Omega}$, respectivamente, e seja $\frac{1}{2}<\alpha \leq 1$. Se $u, v \in L^{\infty}(\Omega) \cap H^{1}(\Omega),\|u\|_{L^{\infty}(\Omega)} \leq R$ e $\|v\|_{L^{\infty}(\Omega)} \leq R$, para alguma constante $R>0$. Então, existe uma constante $L=L(R)>0$ independente de $\epsilon$ tal que

$$
\left\|h^{\epsilon}(u)-h^{\epsilon}(v)\right\|_{H^{-\alpha}(\Omega)} \leq L\|u-v\|_{H^{1}(\Omega)}, \quad 0 \leq \epsilon \leq \epsilon_{0} .
$$

\section{Demonstração:}

Vamos dividir a demonstração em etapas. Primeiramente, para $F$, por definição,

$$
\|F(u)-F(v)\|_{H^{-\alpha}(\Omega)}=\sup _{\substack{\phi \in H^{\alpha}(\Omega) \\\|\phi\|_{H^{\alpha}(\Omega)}=1}}|\langle F(u)-F(v), \phi\rangle| .
$$

Para cada $\phi \in H^{\alpha}(\Omega)$, vamos estimar $|\langle F(u)-F(v), \phi\rangle|$ : 


$$
|\langle F(u)-F(v), \phi\rangle| \leq \int_{\Omega}|(f(x, u(x))-f(x, v(x))) \phi(x)| \mathrm{d} x .
$$

Como $f(x, \cdot): \mathbb{R} \rightarrow \mathbb{R}$ é localmente Lipschitz uniformemente em $x \in \Omega$ e $u \in L^{\infty}(\Omega)$ com $\|u\|_{L^{\infty}(\Omega)} \leq R$, então existe uma constante $L_{1}=L_{1}(R)>0$ tal que

$$
|\langle F(u)-F(v), \phi\rangle| \leq L_{1} \int_{\Omega}|u(x)-v(x)||\phi(x)| \mathrm{d} x .
$$

Usando a desigualdade de Cauchy-Schwartz, obtemos

$$
|\langle F(u)-F(v), \phi\rangle| \leq L_{1}\|u-v\|_{L^{2}(\Omega)}\|\phi\|_{L^{2}(\Omega)} .
$$

Pelo Teorema 2.2.18, $H^{\alpha}(\Omega) \hookrightarrow L^{2}(\Omega)$, com $\frac{1}{2}<\alpha \leq 1$. Logo,

$$
|\langle F(u)-F(v), \phi\rangle| \leq \tilde{L}_{1}\|u-v\|_{H^{1}(\Omega)}\|\phi\|_{H^{\alpha}(\Omega)} .
$$
que

Tomando o supremo com $\|\phi\|_{H^{\alpha}(\Omega)}=1$, obtemos que existe uma constante $L=L(R)>0$ tal

$$
\|F(u)-F(v)\|_{H^{-\alpha}(\Omega)} \leq L\|u-v\|_{H^{1}(\Omega)} .
$$

Agora mostremos para $G^{\epsilon}$. Considere $\epsilon \in\left[0, \epsilon_{0}\right]$, por definição,

$$
\left\|G^{\epsilon}(u)-G^{\epsilon}(v)\right\|_{H^{-\alpha}(\Omega)}=\sup _{\substack{\phi \in H^{\alpha}(\Omega) \\\|\phi\|_{H^{\alpha}(\Omega)}=1}}\left|\left\langle G^{\epsilon}(u)-G^{\epsilon}(v), \phi\right\rangle\right| .
$$

Para cada $\phi \in H^{\alpha}(\Omega)$, vamos estimar $\mid\left\langle G^{\epsilon}(u)-G^{\epsilon}(v), \phi\right\rangle$ :

$$
\left|\left\langle G^{\epsilon}(u)-G^{\epsilon}(v), \phi\right\rangle\right| \leq \frac{1}{\epsilon} \int_{\omega_{\epsilon}}|g(x, u(x))-g(x, v(x))||\phi(x)| \mathrm{d} x .
$$

Como $g(x, \cdot): \mathbb{R} \rightarrow \mathbb{R}$ é localmente Lipschitz uniformemente em $x \in \bar{\Omega}$ e $u \in L^{\infty}(\Omega)$ com $\|u\|_{L^{\infty}(\Omega)} \leq R$, então existe uma constante $L_{2}=L_{2}(R)>0$ tal que

$$
\left|\left\langle G^{\epsilon}(u)-G^{\epsilon}(v), \phi\right\rangle\right| \leq L_{2} \frac{1}{\epsilon} \int_{\omega_{\epsilon}}|u(x)-v(x)||\phi(x)| \mathrm{d} x .
$$

Usando a desigualdade de Cauchy-Schwartz, temos

$$
\left|\left\langle G^{\epsilon}(u)-G^{\epsilon}(v), \phi\right\rangle\right| \leq L_{2}\left(\frac{1}{\epsilon} \int_{\omega_{\epsilon}}|u(x)-v(x)|^{2} \mathrm{~d} x\right)^{\frac{1}{2}}\left(\frac{1}{\epsilon} \int_{\omega_{\epsilon}}|\phi(x)|^{2} \mathrm{~d} x\right)^{\frac{1}{2}},
$$

ou ainda,

$$
\left|\left\langle G^{\epsilon}(u)-G^{\epsilon}(v), \phi\right\rangle\right| \leq L_{2}\left(\frac{1}{\epsilon}\right)^{\frac{1}{2}}\|u-v\|_{L^{2}\left(\omega_{\epsilon}\right)}\left(\frac{1}{\epsilon}\right)^{\frac{1}{2}}\|\phi\|_{L^{2}\left(\omega_{\epsilon}\right)} .
$$

Pelo Lema 2.7.1, $\operatorname{com} q=2$, obtemos que existe uma constante $C>0$, independente de $\epsilon$, tal que

$$
\left|\left\langle G^{\epsilon}(u)-G^{\epsilon}(v), \phi\right\rangle\right| \leq L_{2} C\|u-v\|_{H^{1}(\Omega)}\|\phi\|_{H^{\alpha}(\Omega)} .
$$

Tomando o supremo com $\|\phi\|_{H^{\alpha}(\Omega)}=1$, obtemos que existe uma constante $\tilde{L}=\tilde{L}(R)>0$, independente de $\epsilon$, tal que

$$
\left\|G^{\epsilon}(u)-G^{\epsilon}(v)\right\|_{H^{-\alpha}(\Omega)} \leq \tilde{L}\|u-v\|_{H^{1}(\Omega)}
$$


Para $\epsilon=0$, as contas seguem análogas, contudo, em vez de usar o Lema 2.7.1, usamos que o traço definido em

$$
\gamma: H^{\alpha}(\Omega) \rightarrow L^{2}(\partial \Omega)
$$

é contínuo para $\frac{1}{2}<\alpha \leq 1$. Portanto, segue que existe uma constante $\hat{L}=\hat{L}(R)>0$ tal que

$$
\left\|G^{0}(u)-G^{0}(v)\right\|_{H^{-\alpha}(\Omega)} \leq \hat{L}\|u-v\|_{H^{1}(\Omega)} .
$$

Como usaremos resultados abstratos de comparação, então precisamos de uma ordem nos espaços abstratos que estamos trabalhando. Com base nos resultados de Amann (1995) e pelo Teorema A.13. de Arrieta et al. (2008), podemos afirmar que $H^{1}(\Omega)$ e $H^{-\alpha}(\Omega)$ são espaços de Banach ordenados, respectivamente, com a ordem induzida por $L^{2}(\Omega)$ e a ordem canônica do dual, definidas a seguir.

Definição 3.3.3 (Relação de ordem) Sejam $1<p<\infty, f, g \in L^{p}(\Omega)$ e $\alpha \geq 0$, dizemos que $f \leq g$ em $L^{p}(\Omega)$ se, e somente, se $f(x) \leq g(x)$ para quase todo $x \in \Omega$. E ainda, para $f, g \in\left(H_{p}^{\alpha}(\Omega)\right)^{\prime}$ definimos a ordem canonica do dual por: $f \leq g$ em $\left(H_{p}^{\alpha}(\Omega)\right)^{\prime}$ se $\langle f, \phi\rangle \leq\langle g, \phi\rangle$ para toda $0 \leq \phi \in$ $H_{p}^{\alpha}(\Omega)$.

A seguir, daremos um resultado de comparação para problemas parabólicos com condições de fronteira não linear cuja prova pode ser encontrada no Corolário A.14 em Arrieta et al. (2000).

Lema 3.3.4 (Primeiro critério de comparação) Considere o problema parabólico:

$$
\begin{cases}\frac{\partial w}{\partial t}-\operatorname{div}(a(x) \nabla w)+c(x) w=f(x, w), & (0, \infty) \times \Omega \\ a(x) \frac{\partial w}{\partial \vec{n}}+b(x) w=g(x, w), & (0, \infty) \times \partial \Omega \\ w(0)=w_{0}, & \bar{\Omega}\end{cases}
$$

onde $f, g: \bar{\Omega} \times \mathbb{R} \rightarrow \mathbb{R}$ são funções localmente Lipschitz em $w$ uniformemente em $x \in \Omega$ e $x \in \bar{\Omega}$, respectivamente. Suponha que as funções a,b,c e wo satisfazem condições para o problema (3.15) ser bem posto, por exemplo, em $L^{p}(\Omega)$. Então, enquanto as soluções existirem, temos:

1. Se $w_{0} \geq 0, f(x, 0) \geq 0$ e $g(0, x) \geq 0, \forall x \in \bar{\Omega}$, entẫo $w(t) \geq 0$;

2. Se $w_{1} \geq w_{0}$, então $w\left(t, w_{1}, f, g\right) \geq w\left(t, w_{0}, f, g\right)$;

3. Se $f_{1}(x, w) \geq f_{0}(x, w), g_{1}(x, w) \geq g_{0}(x, w)$ e $w_{1} \geq w_{0}$, então $w\left(t, w_{1}, f_{1}, g_{1}\right) \geq w\left(t, w_{0}, f_{0}, g_{0}\right)$, onde $w\left(t, w_{i}, f_{i}, g_{i}\right)$ denota a solução de (3.15) com condição inicial $w_{i}$ e não linearidades $f_{i}, g_{i}$, $i=1,2$.

Agora, podemos provar o resultado principal desta seção.

Teorema 3.3.5 Assuma que as hipóteses (H1), (H2) e (P) são satisfeitas. Seja $B \subset H^{1}(\Omega)$ um subconjunto limitado e suponha que $u_{0} \in B$, temos:

1. Dado $T>0$, existe uma constante $K(T, B)>0$ independente de $\epsilon$ tal que

$$
\left\|u^{\epsilon}\left(t, u_{0}\right)\right\|_{L^{\infty}(\Omega) \cap H^{1}(\Omega)} \leq K(T, B), \quad \forall t \in[0, T] \quad e \quad 0 \leq \epsilon \leq \epsilon_{0},
$$

onde para cada $0 \leq \epsilon \leq \epsilon_{0}$, u é a solução maximal de (1.1) e (1.4), ou ainda (3.11). 
2. As soluções $u^{\epsilon}$ de (1.1) e (1.4) são globalmente definidas. Em particular, para cada $0 \leq \epsilon \leq \epsilon_{0}$, temos um semigrupo não linear associado a essas soluçôes, dado por -

$$
\begin{aligned}
T^{\epsilon}(t): H^{1}(\Omega) & \rightarrow H^{1}(\Omega) \\
u_{0} & \mapsto T^{\epsilon}(t) u_{0}=u^{\epsilon}\left(t, u_{0}\right) .
\end{aligned}
$$

3. Se (H3) também vale, então existem constantes $K_{\infty}, C_{\infty}>0$ independentes de $\epsilon$ tais que

$$
\lim _{t \rightarrow \infty} \sup \left\|u^{\epsilon}\left(t, u_{0}\right)\right\|_{L^{\infty}(\Omega)} \leq K_{\infty}
$$

$e$

$$
\lim _{t \rightarrow \infty} \sup \left\|u^{\epsilon}\left(t, u_{0}\right)\right\|_{H^{1}(\Omega)} \leq C_{\infty}
$$

e os limites acima são uniformes para $u_{0} \in B$.

4. Se (H3) também vale e, para cada $0 \leq \epsilon \leq \epsilon_{0}$, $\Phi^{\epsilon}$ é a solução dos seguintes problemas elípticos

$$
\begin{cases}-\Delta \Phi^{\epsilon}+(\mu-C(x)) \Phi^{\epsilon}+\frac{1}{\epsilon} \chi_{\omega_{\epsilon}}(x)\left[V_{\epsilon}(x)-E(x)\right] \Phi^{\epsilon}=D(x)+\frac{1}{\epsilon} \chi_{\omega_{\epsilon}}(x) F(x), & \Omega \\ \frac{\partial \Phi^{\epsilon}}{\partial \vec{n}}=0, & \partial \Omega\end{cases}
$$

$e$

$$
\left\{\begin{array}{ll}
-\Delta \Phi^{0}+(\mu-C(x)) \Phi^{0}=D(x), & \Omega \\
\frac{\partial \Phi^{0}}{\partial \vec{n}}+V_{0}(x) \Phi^{0}=E(x) \Phi^{0}+F(x), & \partial \Omega
\end{array} .\right.
$$

Então, $0 \leq \Phi^{\epsilon} \in L^{\infty}(\Omega)$ e $\lim _{t \rightarrow \infty} \sup \left|u^{\epsilon}\left(t, x, u_{0}\right)\right| \leq \Phi^{\epsilon}(x)$, uniformemente em $x \in \bar{\Omega}$ e para $u_{0} \in B$.

\section{Demonstração:}

\section{Item 1.}

Para cada $0 \leq \epsilon \leq \epsilon_{0}$, seja $w_{+}^{\epsilon}\left(t,\left|u_{0}\right|\right)$ as soluções dos problemas parabólicos lineares (3.12) e (3.13).

Uma vez que vale a hipótese (H2), então $F, D \geq 0$. Logo, considerando as funções

$$
\begin{array}{lll}
f_{1}^{\epsilon}(x, w)=C(x) w+D(x)+\frac{1}{\epsilon} \chi_{\omega_{\epsilon}}(x)[E(x) w+F(x)], & x \in \bar{\Omega}, \quad w \in \mathbb{R} \quad \text { e } \quad 0<\epsilon \leq \epsilon_{0} ; \\
f_{1}^{0}(x, w)=C(x) w+D(x), & x \in \bar{\Omega} \text { e } w \in \mathbb{R} ; \\
g_{1}(x, w)=E(x) w+F(x), & x \in \bar{\Omega} \quad \text { e } w \in \mathbb{R} ;
\end{array}
$$

obtemos, para quase todo $x \in \bar{\Omega}$ e $0<\epsilon \leq \epsilon_{0}$

$$
\begin{aligned}
& f_{1}^{\epsilon}(x, 0)=D(x)+\frac{1}{\epsilon} \chi_{\omega_{\epsilon}}(x) F(x) \geq 0 \\
& f_{1}^{0}(x, 0)=D(x) \geq 0 \\
& g_{1}(x, 0)=F(x) \geq 0
\end{aligned}
$$

Além disso, $\left|u_{0}(x)\right| \geq 0$, para quase todo $x \in \Omega$. Logo, pelo item 1. do Lema 3.3.4 temos que $w_{+}^{\epsilon}\left(t,\left|u_{0}\right|\right) \geq 0, \forall 0 \leq \epsilon \leq \epsilon_{0}$.

Agora, $u_{0}(x) \leq\left|u_{0}(x)\right|$, para quase todo $x \in \Omega$, e pela hipótese (H2) sabemos que:

$$
\begin{array}{llll}
f(x, w) \leq f_{1}^{0}(x, w), & \forall x \in \Omega \quad \text { e } \quad w \geq 0 ; \\
g(x, w) \leq g_{1}(x, w), & \forall x \in \bar{\Omega} \quad \text { e } w \geq 0 ; \\
f(x, w)+\frac{1}{\epsilon} \chi_{\omega_{\epsilon}}(x) g(x, w) \leq f_{1}^{\epsilon}(x, w), & \forall x \in \bar{\Omega}, & w \geq 0 \quad \text { e } \quad 0<\epsilon \leq \epsilon_{0} .
\end{array}
$$


Usando o item 3. do Lema 3.3.4, para cada $0 \leq \epsilon \leq \epsilon_{0}$, obtemos que $u^{\epsilon}\left(t, u_{0}\right) \leq w_{+}^{\epsilon}\left(t,\left|u_{0}\right|\right)$ tão longo quanto a solução $u^{\epsilon}\left(t, u_{0}\right)$ existir.

Por outro lado, podemos considerar os problemas parabólicos lineares

$$
\begin{cases}\frac{\partial w^{\epsilon}}{\partial t}+L^{\epsilon} w^{\epsilon}=C(x) w^{\epsilon}-D(x)+\frac{1}{\epsilon} \chi_{\omega_{\epsilon}}(x)\left[E(x) w^{\epsilon}-F(x)\right], & (0, \infty) \times \Omega \\ \frac{\partial w^{\epsilon}}{\partial \vec{n}}=0, & (0, \infty) \times \partial \Omega \\ w^{\epsilon}(0)=-\left|u_{0}\right|, & \bar{\Omega}\end{cases}
$$

onde $L^{\epsilon}$ é dado por (1.2).

$$
\begin{cases}\frac{\partial w^{0}}{\partial t}+L^{0} w^{0}=C(x) w^{0}-D(x), & (0, \infty) \times \Omega, \\ \frac{\partial w^{0}}{\partial \vec{n}}+V_{0}(x) w^{0}=E(x) w^{0}-F(x), & (0, \infty) \times \partial \Omega \\ w^{0}(0)=-\left|u_{0}\right|, & \bar{\Omega}\end{cases}
$$

onde $L^{\epsilon}$ é dado por (1.5).

Seja $w_{-}^{\epsilon}\left(t,-\left|u_{0}\right|\right)$ a solução de (3.18) e (3.19), pela unicidade de soluções, temos que $w_{-}^{\epsilon}\left(t,-\left|u_{0}\right|\right)=$ $-w_{+}^{\epsilon}\left(t,\left|u_{0}\right|\right)$ é a única solução dos problemas (3.18) e (3.19).

Analogamente ao que fizemos anteriormente, definindo as funções

$$
\begin{array}{lll}
f_{2}^{\epsilon}(x, w)=C(x) w-D(x)+\frac{1}{\epsilon} \chi_{\omega_{\epsilon}}(x)[E(x) w-F(x)], & x \in \bar{\Omega}, \quad w \in \mathbb{R} \quad \text { e } 0<\epsilon \leq \epsilon_{0} ; \\
f_{2}^{0}(x, w)=C(x) w-D(x), & x \in \bar{\Omega} \text { e } w \in \mathbb{R} ; \\
g_{2}(x, w)=E(x) w-F(x), & x \in \bar{\Omega} \text { e } w \in \mathbb{R},
\end{array}
$$

e obtemos que $w_{-}^{\epsilon}\left(t,-\left|u_{0}\right|\right) \leq 0,0 \leq \epsilon \leq \epsilon_{0}$.

Agora, $-\left|u_{0}(x)\right| \leq u_{0}(x)$, para quase todo $x \in \Omega$, e pela hipótese (H2) sabemos que:

$$
\begin{array}{llll}
f(x, w) \geq f_{2}^{0}(x, w), & \forall x \in \Omega \quad \text { e } w \leq 0, \\
g(x, w) \geq g_{2}(x, w), & \forall x \in \bar{\Omega} \quad \text { e } w \leq 0, \\
f(x, w)+\frac{1}{\epsilon} \chi_{\omega_{\epsilon}}(x) g(x, w) \geq f_{2}^{\epsilon}(x, w), & \forall x \in \bar{\Omega}, \quad w \leq 0 \quad \text { e } \quad 0<\epsilon \leq \epsilon_{0} .
\end{array}
$$

Assim, de forma análoga, pelo item 3. do Lema 3.3.4 obtemos que $u^{\epsilon}\left(t, u_{0}\right) \geq w_{-}^{\epsilon}\left(t,-\left|u_{0}\right|\right)=$ $-w_{+}^{\epsilon}\left(t,\left|u_{0}\right|\right)$ tão longo quanto $u^{\epsilon}\left(t, u_{0}\right)$ existir.

Portanto, concluímos que $\left|u^{\epsilon}\left(t, u_{0}\right)\right| \leq w_{+}^{\epsilon}\left(t,\left|u_{0}\right|\right)$ tão longo quanto a solução $u^{\epsilon}\left(t, u_{0}\right)$ existir.

Para cada $0 \leq \epsilon \leq \epsilon_{0}$, seja $\Phi^{\epsilon}$ a única solução de (3.16) e (3.17), o qual é a única solução de equilíbrio de (3.12) e (3.13). Pelo resultado de convergência e limitação obtido no Teorema 2.8.1, temos que $\Phi^{\epsilon} \rightarrow \Phi^{0}$ em $L^{\infty}(\Omega)$, quando $\epsilon \rightarrow 0$ e

$$
\left\|\Phi^{\epsilon}\right\|_{L^{\infty}(\Omega)} \leq K, \quad \forall 0 \leq \epsilon \leq \epsilon_{0}
$$

onde $K>0$ independe de $\epsilon$. Além disso, pelo Corolário 2.6 de Rodríguez-Bernal (2011) ou prova do Teorema 3.5 de Jiménes-Casas e Rodríguez-Bernal (2011), $w_{+}^{\epsilon}\left(t,\left|u_{0}\right|\right) \in L^{\infty}(\Omega)$.

Definimos agora

$$
v^{\epsilon}(t, x)=w_{+}^{\epsilon}(t, x)-\Phi^{\epsilon}(x), \quad 0 \leq \epsilon \leq \epsilon_{0},
$$

que satisfaz uma equação parabólica linear semelhante a (3.12) e (3.13), mas com condição inicial

$$
v(0, x)=w_{+}^{\epsilon}(0, x)-\Phi^{\epsilon}(x)=\left|u_{0}(x)\right|-\Phi^{\epsilon}(x), \quad x \in \Omega .
$$

Vamos usar

$$
v^{\epsilon}(t):=v^{\epsilon}\left(t,\left|u_{0}\right|-\Phi^{\epsilon}\right) .
$$


Utilizando a estimativa do semigrupo, (3.6), para $t>0$ temos

$$
\left\|v^{\epsilon}(t)\right\|_{L^{\infty}(\Omega)} \leq M_{3} e^{\beta t} t^{-\frac{n}{4}}\left\|\left|u_{0}\right|-\Phi^{\epsilon}\right\|_{L^{2}(\Omega)} \leq \tilde{M}_{3} e^{\beta t} t^{-\frac{n}{4}}\left(\left\|\left|u_{0}\right|\right\|_{H^{1}(\Omega)}+\left\|\Phi^{\epsilon}\right\|_{L^{\infty}(\Omega)}\right),
$$

para qualquer $-\beta<\lambda_{1}^{0}$, onde $\lambda_{1}$ é o primeiro autovalor do problema elíptico (1.3).

Dado $T>0$ no intervalo maximal de existência de $u^{\epsilon}\left(t, u_{0}\right)$, temos que existe uma constante $K_{1}(T, B, K)>0$, independente de $\epsilon$, tal que

$$
\left\|v^{\epsilon}(t)\right\|_{L^{\infty}(\Omega)} \leq K_{1}(T, B, C), \quad \forall t \in[0, T] .
$$

Portanto, para $t \in[0, T]$,

$$
\left\|w_{+}^{\epsilon}\left(t,\left|u_{0}\right|\right)\right\|_{L^{\infty}(\Omega)} \leq\left\|v^{\epsilon}(t)\right\|_{L^{\infty}(\Omega)}+\left\|\Phi^{\epsilon}\right\|_{L^{\infty}(\Omega)} \leq K_{1}(T, B, K)+K .
$$

Utilizando que $\left|u^{\epsilon}\left(t, u_{0}\right)\right| \leq w_{+}^{\epsilon}\left(t,\left|u_{0}\right|\right)$, obtemos que $u^{\epsilon}\left(t, u_{0}\right) \in L^{\infty}(\Omega)$ e

$$
\left\|u^{\epsilon}\left(t, u_{0}\right)\right\|_{L^{\infty}(\Omega)} \leq K_{2}(T, B, K), \quad \forall t \in[0, T]
$$

onde $K_{2}(T, B, K)>0$ independe de $\epsilon$.

Agora, para $t \in\left[t_{0}, T\right]$. Pela fórmula da variação das constantes

$$
u^{\epsilon}\left(t, u_{0}\right)=e^{-A^{\epsilon} t} u_{0}+\int_{t_{0}}^{t} e^{-A^{\epsilon}(t-s)} h^{\epsilon}\left(u^{\epsilon}\left(s, u_{0}\right)\right) \mathrm{d} s .
$$

Tomando a norma $H^{1}(\Omega)$ e utilizando a estimativa de semigrupo (3.6) com $\frac{1}{2}<\alpha<1$, temos

$$
\left\|u^{\epsilon}\left(t, u_{0}\right)\right\|_{H^{1}(\Omega)} \leq M_{1} e^{\beta t}\left\|u_{0}\right\|_{H^{1}(\Omega)}+\int_{t_{0}}^{t} M_{2} e^{\beta(t-s)}(t-s)^{-\left(\frac{1+\alpha}{2}\right)}\left\|h^{\epsilon}\left(u^{\epsilon}\left(s, u_{0}\right)\right)\right\|_{H^{-\alpha}(\Omega)} \mathrm{d} s,
$$

para qualquer $-\beta<\lambda_{1}$. Além disso, como $u^{\epsilon}\left(t, u_{0}\right) \in H^{1}(\Omega) \cap L^{\infty}(\Omega)$ e vale (3.20), então pelo Lema 3.3.1 existe uma constante $C=C(T, B, K)>0$ que não depende de $\epsilon$ tal que

$$
\left\|h^{\epsilon}\left(u^{\epsilon}\left(s, u_{0}\right)\right)\right\|_{H^{-\alpha}(\Omega)} \leq C, \quad \forall s \in\left[t_{0}, T\right] .
$$

Logo,

$$
\left\|u^{\epsilon}\left(t, u_{0}\right)\right\|_{H^{1}(\Omega)} \leq M_{1} e^{\beta t}\left\|u_{0}\right\|_{H^{1}(\Omega)}+M_{2} C \int_{t_{0}}^{t} e^{\beta(t-s)}(t-s)^{-\left(\frac{1+\alpha}{2}\right)} \mathrm{d} s .
$$

Agora, o lado direito da desigualdade acima é limitado para $t \in\left[t_{0}, T\right]$. Logo, existe uma constante $K(T, B)>0$ independente de $\epsilon$ tal que

$$
\left\|u^{\epsilon}\left(t, u_{0}\right)\right\|_{H^{1}(\Omega)} \leq K(T, B), \quad \forall t \in[0, T] \quad \text { e } \quad \forall 0 \leq \epsilon \leq \epsilon_{0} .
$$

\section{Item 2.}

No item 1., provamos que para cada $0 \leq \epsilon \leq \epsilon_{0}, u^{\epsilon}\left(t, u_{0}\right) \in H^{1}(\Omega) \cap L^{\infty}(\Omega)$ e que a norma de $u^{\epsilon}\left(t, u_{0}\right)$ permanece uniformemente limitada em $H^{1}(\Omega) \cap L^{\infty}(\Omega)$, para $t$ em intervalos de tempo finito. Logo, usando o Corolário 2.5.3 e os Lemas 3.3.1 e 3.3.2, obtemos que as soluções $u^{\epsilon}\left(t, u_{0}\right)$ de (1.1) e (1.4) são definidas para todo $t \geq 0$ e temos um semigrupo não linear em $H^{1}(\Omega)$ dado por $T^{\epsilon}(t) u_{0}=u^{\epsilon}\left(t, u_{0}\right)$.

Note que as constantes obtidas no item 1. poderiam depender de $\epsilon$, pois para a existência global bastaria que para cada $0 \leq \epsilon \leq \epsilon_{0}, u^{\epsilon}\left(t, u_{0}\right)$ permaneça limitada em intervalos de tempo finito.

\section{Item 3.}

Quando a condição (H3) vale, então em (3.16) e (3.17), o operador elíptico é positivo, logo temos um decaimento exponencial do semigrupo linear (como visto na discussão anterior). Mais ainda, se vale (H2), $D, F \geq 0$, e portanto, $\Phi^{\epsilon} \geq 0$. Novamente, tomando $v^{\epsilon}(t, x)=w_{+}^{\epsilon}(t, x)-\Phi^{\epsilon}(x)$ e 
utilizando a estimativa (3.7), temos que

$$
\left\|v^{\epsilon}(t)\right\|_{L^{\infty}(\Omega)} \leq M e^{-\beta t} t^{-\frac{n}{4}}\left(\left\|\left|u_{0}\right|\right\|_{H^{1}(\Omega)}+\left\|\Phi^{\epsilon}\right\|_{L^{\infty}(\Omega)}\right)
$$

para algum $\beta>0$.

Note que $\left\|v^{\epsilon}(t)\right\|_{L^{\infty}(\Omega)} \rightarrow 0$ se $t \rightarrow \infty$. Consequentemente,

$$
\left\|w_{+}^{\epsilon}\left(t,\left|u_{0}\right|\right)\right\|_{L^{\infty}(\Omega)} \leq\left\|v^{\epsilon}(t)\right\|_{L^{\infty}(\Omega)}+\left\|\Phi^{\epsilon}\right\|_{L^{\infty}(\Omega)} \rightarrow\left\|\Phi^{\epsilon}\right\|_{L^{\infty}(\Omega)}, \quad \text { quando } \quad t \rightarrow \infty,
$$

uniformemente para $u_{0} \in B$.

Por outro lado, sabemos que $\left|u^{\epsilon}\left(t, u_{0}\right)\right| \leq w_{+}^{\epsilon}\left(t,\left|u_{0}\right|\right)$. Portanto, existe uma constante $K_{\infty}>0$ independente de $\epsilon$ tal que

$$
\lim _{t \rightarrow \infty} \sup \left\|u^{\epsilon}\left(t, u_{0}\right)\right\|_{L^{\infty}(\Omega)} \leq K_{\infty}
$$

uniformemente para $u_{0} \in B$.

Para $t \geq t_{0}$, onde $t_{0}>0$ é suficientemente grande, tal que

$$
\left\|u^{\epsilon}\left(s, u_{0}\right)\right\|_{L^{\infty}(\Omega)} \leq K_{\infty}+\eta, \quad \forall s \geq t_{0},
$$

para algum $\eta>0$. Agora, utilizando a fórmula da variação das constantes e a estimativa (3.7), $\frac{1}{2}<\alpha<1$, temos

$$
\begin{gathered}
\left\|u^{\epsilon}\left(t, u_{0}\right)\right\|_{H^{1}(\Omega)} \leq\left\|e^{-A^{\epsilon} t} u_{0}\right\|_{H^{1}(\Omega)}+\int_{t_{0}}^{t}\left\|e^{-A^{\epsilon}(t-s)} h^{\epsilon}\left(u^{\epsilon}\left(s, u_{0}\right)\right)\right\|_{H^{1}(\Omega)} \mathrm{d} s \\
\leq M_{1} e^{-\beta t}\left\|u_{0}\right\|_{H^{1}(\Omega)}+M_{2} \int_{t_{0}}^{t} e^{-\beta(t-s)}(t-s)^{-\left(\frac{1+\alpha}{2}\right)}\left\|h^{\epsilon}\left(u^{\epsilon}\left(s, u_{0}\right)\right)\right\|_{H^{-\alpha}(\Omega)} \mathrm{d} s,
\end{gathered}
$$

para algum $\beta>0$.

Pelo Lema 3.3.1, existe uma constante positiva $C>0$ independente de $\epsilon$ tal que

$$
\left\|h^{\epsilon}\left(u^{\epsilon}\left(s, u_{0}\right)\right)\right\|_{H^{-\alpha}(\Omega)} \leq C, \quad \forall s \geq t_{0} .
$$

Logo,

$$
\left\|u^{\epsilon}\left(t, u_{0}\right)\right\|_{H^{1}(\Omega)} \leq M_{1} e^{-\beta t}\left\|u_{0}\right\|_{H^{1}(\Omega)}+M_{2} C \int_{t_{0}}^{t} e^{-\beta(t-s)}(t-s)^{-\left(\frac{1+\alpha}{2}\right)} \mathrm{d} s .
$$

Tomando $w=t-s$ temos que $\mathrm{d} w=-\mathrm{d} s$, se $s=t_{0} \Rightarrow w=t-t_{0}$ e $s=t \Rightarrow w=0$. Substituindo,

$$
\begin{aligned}
\int_{t_{0}}^{t} e^{-\beta(t-s)}(t-s)^{-\left(\frac{1+\alpha}{2}\right)} \mathrm{d} s= & -\int_{t-t_{0}}^{0} e^{-\beta w}(w)^{-\left(\frac{1+\alpha}{2}\right)} \mathrm{d} w=\int_{0}^{t-t_{0}} e^{-\beta w}(w)^{-\left(\frac{1+\alpha}{2}\right)} \mathrm{d} w \\
& <\int_{0}^{\infty} e^{-\beta w}(w)^{-\left(\frac{1+\alpha}{2}\right)} \mathrm{d} w=\beta^{\left(\frac{1+\alpha}{2}\right)-1} \Gamma\left(1-\frac{1+\alpha}{2}\right),
\end{aligned}
$$

onde $\Gamma(z)=\int_{0}^{\infty} x^{z-1} e^{-x} \mathrm{~d} x, \operatorname{com} \operatorname{Re}(z)>0$, é a função Gama. Note que

$$
\frac{1}{2}<\alpha<1 \Rightarrow \frac{3}{4}<\frac{1+\alpha}{2}<1 \Rightarrow 0<1-\frac{1+\alpha}{2}<\frac{1}{4} .
$$

Logo, tomamos $z=1-\frac{1+\alpha}{2}$, temos

$$
\left\|u^{\epsilon}\left(t, u_{0}\right)\right\|_{H^{1}(\Omega)} \leq M_{1} e^{-\beta t}\left\|u_{0}\right\|_{H^{1}(\Omega)}+M_{2} C \beta^{\left(\frac{1+\alpha}{2}\right)-1} \Gamma\left(1-\frac{1+\alpha}{2}\right) .
$$


Portanto, existe uma constante $C_{\infty}>0$ independente de $\epsilon$ tal que

$$
\lim _{t \rightarrow \infty} \sup \left\|u^{\epsilon}\left(t, u_{0}\right)\right\|_{H^{1}(\Omega)} \leq C_{\infty}
$$

independente para $u_{0} \in B$.

Item 4.

Nos itens anteriores, já obtemos que, para cada $0 \leq \epsilon \leq \epsilon_{0}, 0 \leq \Phi^{\epsilon} \in L^{\infty}(\Omega)$. E ainda,

$$
\left|u^{\epsilon}\left(t, x, u_{0}\right)\right| \leq w_{+}^{\epsilon}\left(t, x,\left|u_{0}\right|\right)=v^{\epsilon}\left(t, x,\left|u_{0}\right|-\Phi^{\epsilon}\right)+\Phi^{\epsilon}(x) \leq\left\|v^{\epsilon}(t)\right\|_{L^{\infty}(\Omega)}+\Phi^{\epsilon}(x) .
$$

Como $\left\|v^{\epsilon}(t)\right\|_{L^{\infty}(\Omega)} \rightarrow 0$ se $t \rightarrow \infty$, obtemos que

$$
\lim _{t \rightarrow \infty} \sup \left|u^{\epsilon}\left(t, x, u_{0}\right)\right| \leq \Phi^{\epsilon}(x), \quad \forall x \in \bar{\Omega},
$$

uniformemente para $u_{0} \in B$.

Observação 3.3.6 A limitação uniforme das soluçôes para todo $t \geq 0$ é importante na prova de existência e limitação uniforme dos atratores, para garantirmos que o semigrupo não linear leva conjuntos limitados de $H^{1}(\Omega)$ em órbitas limitadas de $H^{1}(\Omega)$.

Entretanto, apenas a Observação 3.3.6 não é o suficiente para provar a existência de atratores, pois precisamos que o semigrupo não linear seja assintoticamente compacto e, para isso, é necessário mais suavidade. Dessa forma, vamos enunciar um resultado que garante a limitação uniforme das soluções em um espaço um pouco mais regular que $H^{1}(\Omega)$. A prova desse resultado pode ser obtida seguindo os mesmos passos da prova do Teorema 3.3.5, porém trabalhando nas escalas de espaços de Banach construídas anteriormente na Seção 3.1. Essa prova pode ser encontrada no Lema 4.5 em Jiménes-Casas e Rodríguez-Bernal (2011).

Teorema 3.3.7 Assuma que as hipóteses (H1), (H2), (H3) e (P) são satisfeitas. Para qualquer $1<\gamma<1+\frac{1}{2}=\frac{3}{2}$ existe uma constante $K_{\gamma}>0$ independente de $\epsilon$ tal que as soluçôes globais dos problemas (1.1) e (1.4) no Teorema 3.3 .5 satisfazem

$$
\lim _{t \rightarrow \infty} \sup \left\|u^{\epsilon}\left(t, u_{0}\right)\right\|_{H^{\gamma}(\Omega)} \leq K_{\gamma},
$$

e este limite é uniforme para $u_{0} \in B$, onde $B \subset L^{2}(\Omega)$ é um subconjunto limitado. 


\section{Capítulo 4}

\section{Semicontinuidade superior dos atratores e dos equilíbrios}

Neste capítulo vamos provar a existência e semicontinuidade superior da família de atratores $\left\{\mathcal{A}_{\epsilon}\right\}_{\epsilon \in\left[0, \epsilon_{0}\right]}$, em $\epsilon=0$, bem como a semicontinuidade superior da família de equilíbrios $\left\{\mathcal{E}_{\epsilon}\right\}_{\epsilon \in\left[0, \epsilon_{0}\right]}$, em $\epsilon=0$. Para tanto, inicialmente, vamos obter a convergência das soluções, isto é, vamos provar que (1.4) é o problema limite de (1.1). Estaremos assumindo todas as hipóteses (H1), (H2), (H3) e (P) dadas anteriormente.

\subsection{Convergência das soluções}

Vamos provar que as soluções dos problemas concentrado (1.1) convergem para a única solução do problema (1.4) uniformemente em intervalos compactos de $\mathbb{R}^{+}$e para a condição inicial em subconjuntos limitados de $H^{1}(\Omega)$, isto é, a convergência dos semigrupos não lineares,

$$
\left\|T^{\epsilon}(t) u_{0}-T^{0}(t) u_{0}\right\|_{H^{1}(\Omega)} \rightarrow 0, \quad \text { quando } \quad \epsilon \rightarrow 0,
$$

uniformemente para $u_{0} \in B$ e $t \in[0, T]$, onde $B \subset H^{1}(\Omega)$ é limitado e $T>0$. Logo, o problema limite de (1.1) é o problema parabólico (1.4). Para concluir esse resultado, vamos dividir as contas em etapas. Primeiro, vamos mostrar a convergência das não linearidades concentradas.

Lema 4.1.1 Suponha que (H1) seja satisfeita. Se $\|u\|_{H^{1}(\Omega) \cap L^{\infty}(\Omega)} \leq R$, para algum $R>0$, e $\frac{1}{2}<\alpha \leq 1$, então existe $M(\epsilon, R) \rightarrow 0$ quando $\epsilon \rightarrow 0$ tal que

$$
\left\|h^{\epsilon}(u)-h^{0}(u)\right\|_{H^{-\alpha}(\Omega)} \leq M(\epsilon, R)
$$

\section{Demonstração:}

Primeiramente, vamos mostrar o caso $\alpha=1$. Observe que

$$
\left\|h^{\epsilon}(u)-h^{0}(u)\right\|_{H^{-1}(\Omega)}=\left\|\left(F+G^{\epsilon}\right)(u)-\left(F+G^{0}\right)(u)\right\|_{H^{-1}(\Omega)}=\left\|G^{\epsilon}(u)-G^{0}(u)\right\|_{H^{-1}(\Omega)} .
$$

Por definição,

$$
\left\|G^{\epsilon}(u)-G^{0}(u)\right\|_{H^{-1}(\Omega)}=\sup _{\substack{\phi \in H^{1}(\Omega) \\\|\phi\|_{H^{1}(\Omega)}=1}}\left|\left\langle G^{\epsilon}(u)-G^{0}(u), \phi\right\rangle\right|,
$$

onde para cada $\phi \in H^{1}(\Omega)$ e usando (3.9) e (3.10), temos

$$
\left|\left\langle G^{\epsilon}(u)-G^{0}(u), \phi\right\rangle\right|=\left|\frac{1}{\epsilon} \int_{\omega_{\epsilon}} g(x, u(x)) \phi(x) \mathrm{d} x-\int_{\partial \Omega} \gamma(g(x, u(x))) \gamma(\phi(x)) \mathrm{d} S\right| .
$$


Seja $0 \leq \delta<\epsilon$, utilizando as notações da Seção 2.7 temos que $\omega_{\epsilon}=\bigcup_{0 \leq \delta<\epsilon} \Gamma_{\delta}$. Logo, usando $(2.3)$

$$
\left|\left\langle G^{\epsilon}(u)-G^{0}(u), \phi\right\rangle\right|=\left|\frac{1}{\epsilon} \int_{0}^{\epsilon} \int_{\Gamma_{\delta}} g(x, u(x)) \phi(x) \mathrm{d} S_{\delta} \mathrm{d} \delta-\int_{\partial \Omega} \gamma(g(x, u(x))) \gamma(\phi(x)) \mathrm{d} S\right| .
$$

Pela continuidade da aplicação dada no item 1. do Lema 2.7.1, podemos tomar o supremo na igualdade acima e obter

$$
\left|\left\langle G^{\epsilon}(u)-G^{0}(u), \phi\right\rangle\right| \leq \sup _{\delta \in[0, \epsilon]}\left\{\left|\int_{\Gamma_{\delta}} g(x, u(x)) \phi(x) \mathrm{d} S_{\delta}-\int_{\partial \Omega} \gamma(g(x, u(x))) \gamma(\phi(x)) \mathrm{d} S\right|\right\}=\sup _{\delta \in[0, \epsilon]}\{|I(\delta)|\} .
$$

Vamos estimar $|I(\delta)|$.

Fazendo a mudança de variável com o difeomorfismo, $\tau_{\delta}$, definido na Seção 2.7 e usando (2.4), temos

$$
|I(\delta)|=\left|\int_{\partial \Omega} \gamma\left(g\left(\tau_{\delta}(x), u\left(\tau_{\delta}(x)\right)\right)\right) \gamma\left(\phi\left(\tau_{\delta}(x)\right)\right) J\left(\tau_{\delta}(x)\right)-\gamma(g(x, u(x))) \gamma(\phi(x)) \mathrm{d} S\right|,
$$

com $0<J_{1} \leq J\left(\tau_{\delta}(x)\right) \leq J_{2}$ e $\left\|J\left(\tau_{\delta}\right)-I\right\|_{L^{\infty}(\partial \Omega)} \rightarrow 0$ quando $\delta \rightarrow 0, \forall x \in \partial \Omega$ e $\delta \in[0, \epsilon)$. Vamos somar e subtrair dentro da integral em $\partial \Omega$ os termos:

1. $\gamma\left(g\left(\tau_{\delta}(x), u\left(\tau_{\delta}(x)\right)\right)\right) \gamma(\phi(x)) J\left(\tau_{\delta}(x)\right)$;

2. $\gamma\left(g\left(\tau_{\delta}(x), u\left(\tau_{\delta}(x)\right)\right)\right) \gamma(\phi(x))$;

3. $\gamma\left(g\left(\tau_{\delta}(x), u(x)\right)\right) \gamma(\phi(x))$.

Divideremos o problema em 4 integrais, $|I(\delta)|=\left|I_{1}+I_{2}+I_{3}+I_{4}\right|$, onde:

$I_{1}=\int_{\partial \Omega} \gamma\left(g\left(\tau_{\delta}(x), u\left(\tau_{\delta}(x)\right)\right)\right) J\left(\tau_{\delta}(x)\right) \gamma\left(\phi\left(\tau_{\delta}(x)\right)-\phi(x)\right) \mathrm{d} S$;

$I_{2}=\int_{\partial \Omega} \gamma\left(g\left(\tau_{\delta}(x), u\left(\tau_{\delta}(x)\right)\right)\right) \gamma(\phi(x))\left(J\left(\tau_{\delta}(x)\right)-1\right) \mathrm{d} S ;$

$I_{3}=\int_{\partial \Omega} \gamma\left(g\left(\tau_{\delta}(x), u\left(\tau_{\delta}(x)\right)\right)-g\left(\tau_{\delta}(x), u(x)\right)\right) \gamma(\phi(x)) \mathrm{d} S ;$

$I_{4}=\int_{\partial \Omega} \gamma\left(g\left(\tau_{\delta}(x), u(x)\right)-g(x, u(x))\right) \gamma(\phi(x)) \mathrm{d} S$.

Vamos estimar separadamente $\left|I_{1}\right|,\left|I_{2}\right|,\left|I_{3}\right|$ e $\left|I_{4}\right|$.

\section{Estimando $I_{1}$ :}

$$
\left|I_{1}\right| \leq \int_{\partial \Omega}\left|\gamma\left(g\left(\tau_{\delta}(x), u\left(\tau_{\delta}(x)\right)\right)\right)\right|\left|J\left(\tau_{\delta}(x)\right) \| \gamma\left(\phi\left(\tau_{\delta}(x)\right)-\phi(x)\right)\right| \mathrm{d} S .
$$

Como $J_{1} \leq J\left(\tau_{\delta}(x)\right) \leq J_{2}, \forall x \in \partial \Omega, u \in L^{\infty}(\Omega), g$ é uniformemente contínua em $\bar{\Omega} \times \mathbb{R}$ e $\tau_{\delta}$ difeomorfismo, então

$$
\left|I_{1}\right| \leq J_{2}\left\|\gamma\left(g\left(\tau_{\delta}, u\left(\tau_{\delta}\right)\right)\right)\right\|_{L^{\infty}(\partial \Omega)} \int_{\partial \Omega}\left|\gamma\left(\phi\left(\tau_{\delta}(x)\right)-\phi(x)\right)\right| \mathrm{d} S .
$$

Utilizando a desigualdade de Cauchy-Schwartz,

$$
\left|I_{1}\right| \leq J_{2}\left\|\gamma\left(g\left(\tau_{\delta}, u\left(\tau_{\delta}\right)\right)\right)\right\|_{L^{\infty}(\partial \Omega)}|\partial \Omega|^{\frac{1}{2}}\left\|\gamma\left(\phi\left(\tau_{\delta}\right)-\phi\right)\right\|_{L^{2}(\partial \Omega)} .
$$

Pelo Lema 2.7.2 $\operatorname{com} q=2$ :

$$
\left\|\gamma\left(\phi\left(\tau_{\delta}\right)-\phi\right)\right\|_{L^{2}(\partial \Omega)} \leq M \delta^{\frac{1}{2}}\|\nabla \phi\|_{L^{2}\left(\omega_{\delta}\right)} \leq M \delta^{\frac{1}{2}}\|\nabla \phi\|_{L^{2}(\Omega)} \leq M \delta^{\frac{1}{2}}\|\phi\|_{H^{1}(\Omega)} .
$$


E ainda, como $\|u\|_{L^{\infty}(\Omega)} \leq R$, então existe uma constante $L_{1}=L_{1}(R)>0$ tal que

$$
\| \gamma\left(g\left(\tau_{\delta}, u\left(\tau_{\delta}\right)\right) \|_{L^{\infty}(\partial \Omega)} \leq L_{1} .\right.
$$

Usando as considerações acima e para qualquer $\delta<\epsilon$, temos

$$
\left|I_{1}\right| \leq J_{2} L_{1} M \epsilon^{\frac{1}{2}}|\partial \Omega|^{\frac{1}{2}}\|\phi\|_{H^{1}(\Omega)} .
$$

Logo, existe $M_{1}(\epsilon, R) \rightarrow 0$ quando $\epsilon \rightarrow 0$ tal que

$$
\left|I_{1}\right| \leq M_{1}(\epsilon, R)\|\phi\|_{H^{1}(\Omega)} .
$$

\section{Estimando $I_{2}$ :}

$$
\left|I_{2}\right| \leq \int_{\partial \Omega}\left|\gamma\left(g\left(\tau_{\delta}(x), u\left(\tau_{\delta}(x)\right)\right)\right)\right|\left|\gamma(\phi(x)) \| J\left(\tau_{\delta}(x)\right)-1\right| \mathrm{d} S .
$$

De forma análoga à $I_{1}$,

$$
\begin{array}{r}
\left|I_{2}\right| \leq\left\|J\left(\tau_{\delta}\right)-1\right\|_{L^{\infty}(\partial \Omega)}\left\|\gamma\left(g\left(\tau_{\delta}, u\left(\tau_{\delta}\right)\right)\right)\right\|_{L^{\infty}(\partial \Omega)} \int_{\partial \Omega}|\gamma(\phi)| \mathrm{d} S \\
\leq L_{1}\left\|J\left(\tau_{\delta}\right)-1\right\|_{L^{\infty}(\partial \Omega)}|\partial \Omega|^{\frac{1}{2}}\|\gamma(\phi)\|_{L^{2}(\partial \Omega)} .
\end{array}
$$

Pelo Lema 2.2.19, o operador traço $\gamma: H^{1}(\Omega) \rightarrow L^{2}(\partial \Omega)$ é contínuo, logo

$$
\left|I_{2}\right| \leq L_{1}|\partial \Omega|^{\frac{1}{2}}\left\|J\left(\tau_{\delta}\right)-1\right\|_{L^{\infty}(\partial \Omega)}\|\phi\|_{H^{1}(\Omega)} .
$$

Quando $\epsilon \rightarrow 0$ temos que $\delta \rightarrow 0$ e $\left\|J\left(\tau_{\delta}\right)-1\right\|_{L^{\infty}(\partial \Omega)} \rightarrow 0$. Logo, existe $M_{2}(\epsilon, R) \rightarrow 0$ quando $\epsilon \rightarrow 0$ tal que

$$
\left|I_{2}\right| \leq M_{2}(\epsilon, R)\|\phi\|_{H^{1}(\Omega)} .
$$

Estimando $I_{3}$ :

$$
\left|I_{3}\right| \leq \int_{\partial \Omega}\left|\gamma\left(g\left(\tau_{\delta}(x), u\left(\tau_{\delta}(x)\right)\right)-g\left(\tau_{\delta}(x), u(x)\right)\right) \| \gamma(\phi(x))\right| \mathrm{d} S .
$$

Como $g(x, \cdot): \mathbb{R} \rightarrow \mathbb{R}$ é localmente Lipschitz uniformemente em $x \in \bar{\Omega}$ e $u \in L^{\infty}(\Omega)$, com $\|u\|_{L^{\infty}(\Omega)} \leq R$, então existe uma constante $L_{2}=L_{2}(R)>0$ tal que

$$
\left|I_{3}\right| \leq L_{2} \int_{\partial \Omega}\left|\gamma\left(u\left(\tau_{\delta}(x)\right)-u(x)\right)\right||\gamma(\phi(x))| \mathrm{d} S .
$$

Utilizando a desigualdade de Cauchy-Schwartz,

$$
\left|I_{3}\right| \leq L_{2}\left\|\gamma\left(u\left(\tau_{\delta}\right)-u\right)\right\|_{L^{2}(\partial \Omega)}\|\gamma(\phi)\|_{L^{2}(\partial \Omega)} .
$$

Pelo Lema 2.2.19, o traço é um operador contínuo de $H^{1}(\Omega)$ em $L^{2}(\partial \Omega)$, além disso, utilizando o Lema 2.7.2 e para qualquer $\delta<\epsilon$, obtemos

$$
\begin{aligned}
\left|I_{3}\right| \leq L_{2} M \delta^{\frac{1}{2}}\|\nabla u\|_{L^{2}\left(\omega_{\delta}\right)}\|\phi\|_{H^{1}(\Omega)} & \leq L_{2} M \delta^{\frac{1}{2}}\|\nabla u\|_{L^{2}(\Omega)}\|\phi\|_{H^{1}(\Omega)} \\
& \leq L_{2} M \epsilon^{\frac{1}{2}}\|u\|_{H^{1}(\Omega)}\|\phi\|_{H^{1}(\Omega)} .
\end{aligned}
$$

Como $\|u\|_{H^{1}(\Omega)} \leq R$, segue que existe $M_{3}(\epsilon, R) \rightarrow 0$ quando $\epsilon \rightarrow 0$ tal que

$$
\left|I_{3}\right| \leq M_{3}(\epsilon, R)\|\phi\|_{H^{1}(\Omega)} .
$$

Estimando $I_{4}$ : 
De forma análoga as outras integrais,

$$
\left|I_{4}\right| \leq\left\|\gamma\left(g\left(\tau_{\delta}, u\right)-g(\cdot, u)\right)\right\|_{L^{\infty}(\partial \Omega)} \int_{\partial \Omega}|\gamma(\phi(x))| \mathrm{d} S .
$$

Utilizando a desigualdade de Cauchy-Schwartz,

$$
\left|I_{4}\right| \leq\left\|\gamma\left(g\left(\tau_{\delta}, u\right)-g(\cdot, u)\right)\right\|_{L^{\infty}(\partial \Omega)}|\partial \Omega|^{\frac{1}{2}}\|\gamma(\phi)\|_{L^{2}(\partial \Omega)} .
$$

Como o traço é um operador contínuo de $H^{1}(\Omega)$ em $L^{2}(\partial \Omega)$, então

$$
\left|I_{4}\right| \leq\left\|\gamma\left(g\left(\tau_{\delta}, u\right)-g(\cdot, u)\right)\right\|_{L^{\infty}(\partial \Omega)}|\partial \Omega|^{\frac{1}{2}}\|\phi\|_{H^{1}(\Omega)} .
$$

Como $g$ é uniformemente contínua na primeira variável, $\tau_{\delta}$ é um difeomorfismo de classe $C^{2}$ com $\left\|\tau_{\delta}-I\right\|_{C^{2}(\bar{\Omega})} \rightarrow 0$ quando $\delta \rightarrow 0$, mas se $\delta \rightarrow 0$ segue que $\epsilon \rightarrow 0$. Logo, existe $M_{4}(\epsilon, R) \rightarrow 0$ quando $\epsilon \rightarrow 0$ tal que

$$
\left|I_{4}\right| \leq M_{4}(\epsilon, R)\|\phi\|_{H^{1}(\Omega)}
$$

Substituindo em $I(\delta)$,

$$
|I(\delta)| \leq\left(M_{1}(\epsilon, R)+M_{2}(\epsilon, R)+M_{3}(\epsilon, R)+M_{4}(\epsilon, R)\right)\|\phi\|_{H^{1}(\Omega)}=M(\epsilon, R)\|\phi\|_{H^{1}(\Omega)},
$$

com $M(\epsilon, R) \rightarrow 0$ quando $\epsilon \rightarrow 0$. Portanto, para qualquer $\phi \in H^{1}(\Omega)$,

$$
\left|\left\langle G^{\epsilon}(u)-G^{0}(u), \phi\right\rangle\right| \leq M(\epsilon, R)\|\phi\|_{H^{1}(\Omega)} .
$$

Tomando o supremo de $\|\phi\|_{H^{1}(\Omega)}=1$, obtemos

$$
\left\|G^{\epsilon}(u)-G^{0}(u)\right\|_{H^{-1}(\Omega)} \leq M(\epsilon, R),
$$

onde $M(\epsilon, R) \rightarrow 0$ quando $\epsilon \rightarrow 0$.

Agora, vamos mostrar que o resultado ainda vale para qualquer $\alpha$ tal que $\frac{1}{2}<\alpha<1$ usando inteporlação. Inicialmente, note que $\left\|h^{\epsilon}(u)-h^{0}(u)\right\|_{H^{-\alpha}(\Omega)}=\left\|G^{\epsilon}(u)-G^{0}(u)\right\|_{H^{-\alpha}(\Omega)}$. Seja $0 \leq$ $\theta \leq 1$ e $\frac{1}{2}<\alpha_{0}<1$ fixo tal que $-1<-\alpha<-\alpha_{0}<-\frac{1}{2}$. Pelo Teorema 2.2.15, temos

$$
\left\|G^{\epsilon}(u)-G^{0}(u)\right\|_{H^{-\alpha}(\Omega)} \leq\left\|G^{\epsilon}(u)-G^{0}(u)\right\|_{H^{-\alpha_{0}(\Omega)}}^{\theta}\left\|G^{\epsilon}(u)-G^{0}(u)\right\|_{H^{-1}(\Omega)}^{1-\theta} .
$$

Pelo Lema 3.3.1, $\left\|G^{\epsilon}(u)-G^{0}(u)\right\|_{H^{-\alpha_{0}(\Omega)}} \leq C$, onde $C=C(R)>0$ independe de $\epsilon$. Usando (4.1), obtemos

$$
\left\|G^{\epsilon}(u)-G^{0}(u)\right\|_{H^{-\alpha}(\Omega)} \leq M(\epsilon, R)
$$

onde $M(\epsilon, R) \rightarrow 0$ quando $\epsilon \rightarrow 0$.

Assim, concluimos a demonstração.

E finalmente, vamos demonstrar a convergência dos semigrupos não lineares, ou ainda, que o problema limite de (1.1) é dado por (1.4). Porém, para isso é importante destacar que a convergência dos operadores resolventes $\left(A^{\epsilon}\right)^{-1}$ para $\left(A^{0}\right)^{-1}$ já foi provado no Corolário 4.2 em Arrieta et al. (2008). Usando isso e o Teorema 2.3.9 é possível obter a convergência do semigrupo linear $e^{-A^{\epsilon} t}$ para $e^{-A^{0} t}$, como enunciaremos a seguir (ver Lema 2.5. de Jiménes-Casas e Rodríguez-Bernal (2009) ou Teorema 2.3 em Jiménes-Casas e Rodríguez-Bernal (2011)). 
Lema 4.1.2 Suponha que vale a hipótese (P). Seja $\frac{1}{2}<\alpha<1$ e $T>0$ qualquer. Então, existe uma função positiva $C(\epsilon) \rightarrow 0$ quando $\epsilon \rightarrow 0$ tal que, para toda $h \in H^{-\alpha}(\Omega)$, temos

$$
\left\|e^{-A^{\epsilon} t} h-e^{-A^{0} t} h\right\|_{H^{1}(\Omega)} \leq C(\epsilon) t^{-\left(\frac{1+\alpha}{2}\right)}\|h\|_{H^{-\alpha}(\Omega)}, \quad t \in(0, T] .
$$

Teorema 4.1.3 (Convergência das soluções) Suponha que as hipóteses (H1), (H2), (H3) e (P) são satisfeitas. Sejam $T>0, \frac{1}{2}<\alpha<1, B \subset H^{1}(\Omega)$ um subconjunto limitado e $u_{0} \in B$. Então, existe uma função positiva $C(\stackrel{T}{T}, B, \epsilon) \rightarrow 0$ quando $\epsilon \rightarrow 0$, tal que

$$
\left\|T^{\epsilon}(t) u_{0}-T^{0}(t) u_{0}\right\|_{H^{1}(\Omega)} \leq C(T, B, \epsilon) t^{-\left(\frac{1+\alpha}{2}\right)}, \quad \forall t \in(0, T] .
$$

Em particular,

$$
T^{\epsilon}(t) u_{0} \rightarrow T^{0}(t) u_{0} \quad \text { em } \quad H^{1}(\Omega), \quad \text { quando } \quad \epsilon \rightarrow 0,
$$

uniformemente para $u_{0} \in B$ e $t \in[0, T]$.

\section{Demonstração:}

Sabemos que sob essas hipóteses $u^{\epsilon}\left(t, u_{0}\right)=T^{\epsilon}(t) u_{0}$ e $u^{0}\left(t, u_{0}\right)=T^{0}(t) u_{0}$ são globalmente definidas e uniformemente limitadas em $H^{1}(\Omega) \cap L^{\infty}(\Omega)$ em intervalos compactos de $\mathbb{R}^{+}$(ver Teorema 3.3.5). Pela fórmula da variação das constantes, obtemos que, para $0<\epsilon \leq \epsilon_{0}$ e $t \in(0, T]$,

$$
\begin{gathered}
\left\|T^{\epsilon}(t) u_{0}-T^{0}(t) u_{0}\right\|_{H^{1}(\Omega)}=\|\left(e^{-A^{\epsilon} t}-e^{-A^{0} t}\right) u_{0} \\
+\int_{0}^{t}\left[e^{-A^{\epsilon}(t-s)} h^{\epsilon}\left(T^{\epsilon}(s) u_{0}\right)-e^{-A^{0}(t-s)} h^{0}\left(T^{0}(s) u_{0}\right)\right] \mathrm{d} s \|_{H^{1}(\Omega)} .
\end{gathered}
$$

Pela desigualdade triangular,

$$
\left\|T^{\epsilon}(t) u_{0}-T^{0}(t) u_{0}\right\|_{H^{1}(\Omega)} \leq N_{1}+\int_{0}^{t} N_{2} \mathrm{~d} s
$$

onde

$$
N_{1}=\left\|e^{-A^{\epsilon} t} u_{0}-e^{-A^{0} t} u_{0}\right\|_{H^{1}(\Omega)} \quad \text { e } \quad N_{2}=\left\|e^{-A^{\epsilon}(t-s)} h^{\epsilon}\left(T^{\epsilon}(s) u_{0}\right)-e^{-A^{0}(t-s)} h^{0}\left(T^{0}(s) u_{0}\right)\right\|_{H^{1}(\Omega)} \text {. }
$$

Vamos estimar $N_{1}$ e $N_{2}$.

\section{Caso $N_{1}$}

Aplicando o Lema 4.1.2, existe uma função positiva $C(\epsilon) \rightarrow 0$ quando $\epsilon \rightarrow 0$ tal que

$$
\left\|e^{-A^{\epsilon} t} u_{0}-e^{-A^{0} t} u_{0}\right\|_{H^{1}(\Omega)} \leq C(\epsilon) t^{-\left(\frac{1+\alpha}{2}\right)}\left\|u_{0}\right\|_{H^{-\alpha}(\Omega)}, \quad \forall t \in(0, T] .
$$

Pela imersão $H^{1}(\Omega) \hookrightarrow H^{-\alpha}(\Omega)$ e por $u_{0} \in B$, existe $C_{1}(B, \epsilon) \rightarrow 0$, quando $\epsilon \rightarrow 0$, tal que

$$
N_{1} \leq C_{1}(B, \epsilon) t^{-\left(\frac{1+\alpha}{2}\right)}, \quad \forall t \in(0, T] .
$$

Caso $\mathrm{N}_{2}$

Somando e subtraindo os termos

- $e^{-A^{\epsilon}(t-s)} h^{0}\left(T^{\epsilon}(s) u_{0}\right)$;

- $e^{-A^{0}(t-s)} h^{0}\left(T^{\epsilon}(s) u_{0}\right)$.

Dividimos $N_{2}$ em 3 parcelas $N_{2} \leq I_{1}+I_{2}+I_{3}$, onde

1. $I_{1}=\left\|e^{-A^{\epsilon}(t-s)} h^{\epsilon}\left(T^{\epsilon}(s) u_{0}\right)-e^{-A^{\epsilon}(t-s)} h^{0}\left(T^{\epsilon}(s) u_{0}\right)\right\|_{H^{1}(\Omega)}$; 
2. $I_{2}=\left\|e^{-A^{\epsilon}(t-s)} h^{0}\left(T^{\epsilon}(s) u_{0}\right)-e^{-A^{0}(t-s)} h^{0}\left(T^{\epsilon}(s) u_{0}\right)\right\|_{H^{1}(\Omega)}$;

3. $I_{3}=\left\|e^{-A^{0}(t-s)} h^{0}\left(T^{\epsilon}(s) u_{0}\right)-e^{-A^{0}(t-s)} h^{0}\left(T^{0}(s) u_{0}\right)\right\|_{H^{1}(\Omega)}$.

Vamos estimar $I_{1}$ : usando a estimativa do semigrupo (3.7) para $t \in(0, T]$ e $s \in[0, t]$, temos

$$
\begin{gathered}
I_{1} \leq\left\|e^{-A^{\epsilon}(t-s)}\left[h^{\epsilon}\left(T^{\epsilon}(s) u_{0}\right)-h^{0}\left(T^{\epsilon}(s) u_{0}\right)\right]\right\|_{H^{1}(\Omega)} \\
\Rightarrow I_{1} \leq M_{2} e^{-\beta(t-s)}(t-s)^{-\left(\frac{1+\alpha}{2}\right)}\left\|h^{\epsilon}\left(T^{\epsilon}(s) u_{0}\right)-h^{0}\left(T^{\epsilon}(s) u_{0}\right)\right\|_{H^{-\alpha}(\Omega)} .
\end{gathered}
$$

Pelo Teorema 3.3.5 existe $K(T, B)>0$ independente de $\epsilon$ tal que

$$
\left\|T^{\epsilon}(s) u_{0}\right\|_{H^{1}(\Omega) \cap L^{\infty}(\Omega)} \leq K(T, B), \quad \forall s \in[0, T] \quad \text { e } \quad 0 \leq \epsilon \leq \epsilon_{0} .
$$

Agora, usando o Lema 4.1.1, existe $M(T, B, \epsilon) \rightarrow 0$, quando $\epsilon \rightarrow 0$, tal que

$$
\left\|h^{\epsilon}\left(T^{\epsilon}(s) u_{0}\right)-h^{0}\left(T^{\epsilon}(s) u_{0}\right)\right\|_{H^{-\alpha}(\Omega)} \leq M(T, B, \epsilon) .
$$

Portanto, para $t \in(0, T]$ e $s \in[0, t]$,

$$
I_{1} \leq M_{2} M(T, B, \epsilon) e^{-\beta(t-s)}(t-s)^{-\left(\frac{1+\alpha}{2}\right)} .
$$

Vamos estimar $I_{2}$ : usando a convergência do semigrupo linear (Lema 4.1.2), existe $C(\epsilon) \rightarrow 0$, quando $\epsilon \rightarrow 0$, tal que para $t \in(0, T]$ e $s \in[0, t]$, temos

$$
I_{2} \leq\left\|\left[e^{-A^{\epsilon}(t-s)}-e^{-A^{0}(t-s)}\right] h^{0}\left(T^{\epsilon}(s) u_{0}\right)\right\|_{H^{1}(\Omega)} \leq C(\epsilon)(t-s)^{-\left(\frac{1+\alpha}{2}\right)}\left\|h^{0}\left(T^{\epsilon}(s) u_{0}\right)\right\|_{H^{-\alpha}(\Omega)} .
$$

Usando (4.2) e o Lema 3.3.1, temos que existe $C(T, B)>0$, independente de $\epsilon$, tal que

$$
\left\|h^{0}\left(T^{\epsilon}(s) u_{0}\right)\right\|_{H^{-\alpha}(\Omega)} \leq C(T, B), \quad \forall s \in[0, T] .
$$

Portanto, para $t \in(0, T]$ e $s \in[0, t]$, temos

$$
I_{2} \leq C(\epsilon) C(T, B)(t-s)^{-\left(\frac{1+\alpha}{2}\right)} .
$$

Vamos estimar $I_{3}$ : usando a estimativa do semigrupo (3.7), para $t \in(0, T]$ e $s \in[0, t]$, temos

$$
\begin{aligned}
I_{3} & \leq\left\|e^{-A^{0}(t-s)}\left[h^{0}\left(T^{\epsilon}(s) u_{0}\right)-h^{0}\left(T^{0}(s) u_{0}\right)\right]\right\|_{H^{1}(\Omega)} \\
& \leq M_{2} e^{-\beta(t-s)}(t-s)^{-\left(\frac{1+\alpha}{2}\right)}\left\|h^{0}\left(T^{\epsilon}(s) u_{0}\right)-h^{0}\left(T^{0}(s) u_{0}\right)\right\|_{H^{-\alpha}(\Omega)} .
\end{aligned}
$$

Agora por (4.2) e usando o Lema 3.3.2, obtemos que existe uma constante $L(T, B)>0$ independente de $\epsilon$ tal que

$$
\left\|h^{0}\left(T^{\epsilon}(s) u_{0}\right)-h^{0}\left(T^{0}(s) u_{0}\right)\right\|_{H^{-\alpha}(\Omega)} \leq L(T, B)\left\|T^{\epsilon}(s) u_{0}-T^{0}(s) u_{0}\right\|_{H^{1}(\Omega)} .
$$

Portanto, para $t \in(0, T]$ e $s \in[0, t]$, temos

$$
I_{3} \leq M_{2} L(T, B) e^{-\beta(t-s)}(t-s)^{-\left(\frac{1+\alpha}{2}\right)}\left\|T^{\epsilon}(s) u_{0}-T^{0}(s) u_{0}\right\|_{H^{1}(\Omega)} .
$$

Usando todas as estimativas, para $t \in(0, T]$, e o fato de $e^{-\beta(t-s)}<1$, obtemos 


$$
\begin{array}{r}
\left\|T^{\epsilon}(s) u_{0}-T^{0}(s) u_{0}\right\|_{H^{1}(\Omega)} \leq C_{1}(B, \epsilon) t^{-\left(\frac{1+\alpha}{2}\right)}+\left[M_{2} M(T, B, \epsilon)+C(\epsilon) C(T, B)\right] \int_{0}^{t}(t-s)^{-\left(\frac{1+\alpha}{2}\right)} \mathrm{d} s \\
+M_{2} L(T, B) \int_{0}^{t}(t-s)^{-\left(\frac{1+\alpha}{2}\right)}\left\|T^{\epsilon}(s) u_{0}-T^{0}(s) u_{0}\right\|_{H^{1}(\Omega)} \mathrm{d} s .
\end{array}
$$

Note que, $t \in(0, T]$,

$$
\int_{0}^{t}(t-s)^{-\left(\frac{1+\alpha}{2}\right)} \mathrm{d} s=\frac{t^{1-\left(\frac{1+\alpha}{2}\right)}}{1-\left(\frac{1+\alpha}{2}\right)}=\frac{t}{1-\left(\frac{1+\alpha}{2}\right)} t^{-\left(\frac{1+\alpha}{2}\right)} \leq \frac{T}{1-\left(\frac{1+\alpha}{2}\right)} t^{-\left(\frac{1+\alpha}{2}\right)} .
$$

Logo, existem constantes positivas $C_{2}(B, \epsilon)$ e $C_{3}(T, B)$, com $C_{2}(B, \epsilon) \rightarrow 0$, quando $\epsilon \rightarrow 0$, tais que

$$
\left\|T^{\epsilon}(s) u_{0}-T^{0}(s) u_{0}\right\|_{H^{1}(\Omega)} \leq C_{2}(B, \epsilon) t^{-\left(\frac{1+\alpha}{2}\right)}+C_{3}(T, B) \int_{0}^{t}(t-s)^{-\left(\frac{1+\alpha}{2}\right)}\left\|T^{\epsilon}(s) u_{0}-T^{0}(s) u_{0}\right\|_{H^{1}(\Omega)} \mathrm{d} s .
$$

Aplicando a desigualdade de Gronwall (Lema 2.5.5), temos que existe uma função positiva $C(T, B, \epsilon) \rightarrow 0$, quando $\epsilon \rightarrow 0$, tal que

$$
\left\|T^{\epsilon}(t) u_{0}-T^{0}(t) u_{0}\right\|_{H^{1}(\Omega)} \leq C(T, B, \epsilon) t^{-\left(\frac{1+\alpha}{2}\right)}, \quad \forall t \in(0, T] .
$$

Em particular,

$$
T^{\epsilon}(t) u_{0} \rightarrow T^{0}(t) u_{0} \quad \text { em } \quad H^{1}(\Omega), \quad \text { quando } \quad \epsilon \rightarrow 0,
$$

uniformemente para $u_{0} \in B$ e $t \in[0, T]$.

Por fim, note que o caso $t=0$ é imediato, pois $T^{\epsilon}(0) u_{0}=u_{0}=T^{0}(0) u_{0}$.

Observação 4.1.4 Como o resultado de convergência no Teorema 4.1 .3 é obtido em intervalos compactos de $\mathbb{R}^{+}$, então podemos também provar o resultado sem a hipótese (H3), para tanto, basta usar as estimativas do semigrupo (3.6) e os itens 1 e 2 do Teorema 3.3.5.

\subsection{Existência e semicontinuidade superior dos atratores globais}

Nesta seção, mostraremos a existência de uma família de atratores globais dos problemas (1.1) e (1.4) e sua semicontinuidade superior.

Para mostrarmos a existência dos atratores é suficiente que o semigrupo $\left\{T^{\epsilon}(t): t \geq 0\right\}$ seja assintoticamente compacto, ponto dissipativo e eventualmente limitado em $H^{1}(\Omega)$ (ver Teorema 2.6.9). Para tanto, é essencial assumirmos a condição de dissipatividade (H3) para garantirmos a limitação uniforme das soluções de (1.1) e (1.4) em $H^{\gamma}(\Omega)$, com $1 \leq \gamma<\frac{3}{2}$, para todo o tempo (vide os Teoremas 3.3.5 e 3.3.7).

A seguir, provamos a existência dos atratores e sua limitação uniforme em $H^{1}(\Omega) \cap L^{\infty}(\Omega)$.

Teorema 4.2.1 (Existência e limitação dos atratores) Suponha que valem as hipóteses (H1), (H2), (H3) e (P). Então, para cada $\epsilon \in\left[0, \epsilon_{0}\right]$, os problemas (1.1) e (1.4) possuem um atrator global $\mathcal{A}_{\epsilon}$ em $H^{1}(\Omega)$.

Além disso, existe $K>0$ independente de $\epsilon$ tal que

$$
\sup _{\epsilon \in\left[0, \epsilon_{0}\right]} \sup _{u^{\epsilon} \in \mathcal{A}_{\epsilon}}\left\|u^{\epsilon}\right\|_{H^{1}(\Omega) \cap L^{\infty}(\Omega)} \leq K .
$$

Em particular, $\mathcal{A}_{0}$ atrai $\bigcup_{0<\epsilon \leq \epsilon_{0}} \mathcal{A}_{\epsilon}$ em $H^{1}(\Omega)$. 


\section{Demonstração:}

Vamos dividir a demonstração em etapas:

\section{Parte I - Eventualmente limitado.}

Pelo Teorema 3.3.5 sabemos que o semigrupo não linear $T^{\epsilon}(t)$ leva conjuntos limitados de $H^{1}(\Omega)$ em limitados de $H^{1}(\Omega)$, para todo $\epsilon \in\left[0, \epsilon_{0}\right]$. Logo, a órbita positiva de conjuntos limitados de $H^{1}(\Omega)$ é limitada em $H^{1}(\Omega)$ e concluímos que o semigrupo não linear é eventualmente limitado.

\section{Parte II - Ponto dissipativo.}

Na verdade, vamos mostrar que, para cada $\epsilon \in\left[0, \epsilon_{0}\right], T^{\epsilon}(t)$, é limitado dissipativo em $H^{1}(\Omega)$, isto é, existe um subconjunto limitado $B \subset H^{1}(\Omega)$ que atrai subconjuntos limitados de $H^{1}(\Omega)$ por $T^{\epsilon}(t)$.

Considere $B \subset H^{1}(\Omega)$ um subconjunto limitado qualquer e $u_{0} \in B$, pelo Teorema 3.3.5 sabemos que existe $C_{\infty}>0$ independente de $\epsilon$ tal que

$$
\lim _{t \rightarrow \infty} \sup \left\|u^{\epsilon}\left(t, u_{0}\right)\right\|_{H^{1}(\Omega)} \leq C_{\infty}, \quad \forall 0 \leq \epsilon \leq \epsilon_{0},
$$

uniformemente em $u_{0} \in B$.

Assim, para algum $t_{0}>0$ suficientemente grande, existe $\eta>0$ tal que

$$
\left\|u^{\epsilon}\left(t, u_{0}\right)\right\|_{H^{1}(\Omega)} \leq C_{\infty}+\eta, \quad \forall t \geq t_{0}, \quad \forall 0 \leq \epsilon \leq \epsilon_{0} \quad \text { e } \quad \forall u_{0} \in B .
$$

Tomando a bola fechada em $H^{1}(\Omega)$ centrada em zero e raio $C_{\infty}+\eta>0$,

$$
\overline{B_{C_{\infty}+\eta}}(0)=\left\{u \in H^{1}(\Omega):\|u\|_{H^{1}(\Omega)} \leq C_{\infty}+\eta\right\}
$$

é claro que para $t \geq t_{0}, T^{\epsilon}(t) u_{0} \in \overline{B_{C_{\infty}+\eta}}(0), \forall u_{0} \in B$. Logo, $\overline{B_{C_{\infty}+\eta}}(0)$ absorve $B$ e, consequentemente também atrai $B$. Portanto, $T^{\epsilon}(t)$ é limitado dissipativo em $H^{1}(\Omega)$.

\section{Parte III - Assintoticamente compacto.}

Na verdade, vamos provar que o semigrupo é condicionalmente eventualmente compacto.

Dado um subconjunto $B \subset H^{1}(\Omega)$ limitado, temos pelo Teorema 3.3.7 que, para algum $t_{0}>0$ suficientemente grande e $1<\gamma<\frac{3}{2}$ fixo, existem $\eta>0$ e $C_{\gamma}>0$ independentes de $\epsilon$ tais que

$$
\sup _{\epsilon \in\left[0, \epsilon_{0}\right]} \sup _{u_{0} \in B}\left\|u^{\epsilon}\left(t, u_{0}\right)\right\|_{H^{\gamma}(\Omega)} \leq C_{\gamma}+\eta, \quad \forall t \geq t_{0},
$$

o que implica que $T^{\epsilon}(t) B$ é um conjunto limitado em $H^{\gamma}(\Omega), \forall t \geq t_{0}$.

Agora, pelo Teorema 2.2.18, a imersão $H^{\gamma}(\Omega) \hookrightarrow H^{1}(\Omega)$ é compacta. Portanto, $T^{\epsilon}(t) B$ é relativamente compacto em $H^{1}(\Omega)$, para todo $t \geq t_{0}$, isto é, $\overline{T^{\epsilon}(t) B}$ é compacto em $H^{1}(\Omega)$, para todo $t \geq t_{0}$.

Portanto, o semigrupo $\left\{T^{\epsilon}(t): t \geq 0\right\}$ é condicionalmente eventualmente compacto. Agora, usando o Teorema 2.6.8, segue que $\left\{T^{\epsilon}(t): t \geq 0\right\}$ é assintoticamente compacto.

Com isso concluímos a existência dos atratores globais $\mathcal{A}_{\epsilon}$, para cada $\epsilon \in\left[0, \epsilon_{0}\right]$, dos problemas (1.1) e (1.4).

Parte IV - Limitação uniforme dos atratores em $H^{1}(\Omega) \cap L^{\infty}(\Omega)$.

Segue do Teorema 3.3.5, que existem constantes $K_{\infty}, C_{\infty}>0$ independentes de $\epsilon$ tais que

$$
\lim _{t \rightarrow \infty} \sup \left\|u^{\epsilon}\left(t, u_{0}\right)\right\|_{L^{\infty}(\Omega)} \leq K_{\infty} \quad \text { e } \quad \lim _{t \rightarrow \infty} \sup \left\|u^{\epsilon}\left(t, u_{0}\right)\right\|_{H^{1}(\Omega)} \leq C_{\infty}
$$

onde os limites são uniformes para $u_{0}$ em subconjuntos limitados de $H^{1}(\Omega)$. Logo, como na parte II, existe um conjunto absorvente uniformemente limitado em $L^{\infty}(\Omega)$ e $H^{1}(\Omega)$. 
Agora, como, para cada $\epsilon \in\left[0, \epsilon_{0}\right]$, o atrator $\mathcal{A}_{\epsilon}$ é limitado e invariante para $T^{\epsilon}(t)$, isto é, $T^{\epsilon}(t) \mathcal{A}_{\epsilon}=\mathcal{A}_{\epsilon}, t \geq 0$, então segue a limitação uniforme dos atratores em $H^{1}(\Omega) \cap L^{\infty}(\Omega)$.

Além disso, $\bigcup_{0<\epsilon \leq \epsilon_{0}} \mathcal{A}_{\epsilon} \subset H^{1}(\Omega)$ é uniformemente limitado e $\mathcal{A}_{0}$ é atrator global para $T^{0}(t)$, logo $\mathcal{A}_{0}$ atrai cada subconjunto limitado de $H^{1}(\Omega)$, em particular, $\mathcal{A}_{0}$ atrai $\bigcup_{0<\epsilon \leq \epsilon_{0}} \mathcal{A}_{\epsilon}$ em $H^{1}(\Omega)$.

Na Seção 4.1, obtemos um resultado importante, provamos que as soluções de (1.1) convergem para a única solução de $(1.4)$ em $H^{1}(\Omega)$ uniformemente para condição inicial $u_{0}$ em limitados de $H^{1}(\Omega)$ e $t$ em intervalos compactos de $\mathbb{R}^{+}$. Contudo, para concluir que a família de atratores $\left\{\mathcal{A}_{\epsilon}\right\}_{\epsilon \in\left[0, \epsilon_{0}\right]}$ de (1.1) e (1.4) é semicontínua superiormente em $\epsilon=0$ precisamos de um resultado parecido da convergência do semigrupo não linear sobre os elementos dos atratores, que será dado a seguir.

Lema 4.2.2 Suponha que as hipóteses (H1), (H2), (H3) e (P) são satisfeitas. Sejam $T>0, \frac{1}{2}<$ $\alpha<1$ e $\mathcal{A}_{\epsilon}, 0<\epsilon \leq \epsilon_{0}$, o atrator de (1.1) dado no Teorema 4.2.1. Então, se $u^{\epsilon} \in \mathcal{A}_{\epsilon}, 0<\epsilon \leq \epsilon_{0}$, existe uma constante positiva $C(T, \epsilon) \rightarrow 0$ quando $\epsilon \rightarrow 0$ tal que

$$
\left\|T^{\epsilon}(t) u^{\epsilon}-T^{0}(t) u^{\epsilon}\right\|_{H^{1}(\Omega)} \leq C(T, \epsilon) t^{-\left(\frac{1+\alpha}{2}\right)}, \quad \forall t \in(0, T] .
$$

\section{Demonstração:}

Esta demonstração é técnicamente parecida com a demonstração do Teorema 4.1.3 mudando apenas algumas justificativas, portanto, alguns passos que já foram realizados no Teorema 4.1.3 serão omitidos.

Sejam $u^{\epsilon} \in \mathcal{A}_{\epsilon}, 0<\epsilon \leq \epsilon_{0}$, e $T>0$, pela fórmula da variação das constantes, obtemos que, para $t \in(0, T]$,

$$
\begin{gathered}
\left\|T^{\epsilon}(t) u^{\epsilon}-T^{0}(t) u^{\epsilon}\right\|_{H^{1}(\Omega)}=\| e^{-A^{\epsilon} t} u^{\epsilon}-e^{-A^{0} t} u^{\epsilon} \\
+\int_{0}^{t}\left[e^{-A^{\epsilon}(t-s)} h^{\epsilon}\left(T^{\epsilon}(s) u^{\epsilon}\right)-e^{-A^{0}(t-s)} h^{0}\left(T^{0}(s) u^{\epsilon}\right)\right] \mathrm{d} s \|_{H^{1}(\Omega)} .
\end{gathered}
$$

Pela desigualdade triangular,

$$
\left\|T^{\epsilon}(t) u^{\epsilon}-T^{0}(t) u^{\epsilon}\right\|_{H^{1}(\Omega)} \leq N_{1}+\int_{0}^{t} N_{2} \mathrm{~d} s
$$

onde

$$
N_{1}=\left\|e^{-A^{\epsilon} t} u^{\epsilon}-e^{-A^{0} t} u^{\epsilon}\right\|_{H^{1}(\Omega)} \quad \text { e } \quad N_{2}=\left\|e^{-A^{\epsilon}(t-s)} h^{\epsilon}\left(T^{\epsilon}(s) u^{\epsilon}\right)-e^{-A^{0}(t-s)} h^{0}\left(T^{0}(s) u^{\epsilon}\right)\right\|_{H^{1}(\Omega)} .
$$

Vamos estimar $N_{1}$ e $N_{2}$.

\section{Caso $N_{1}$}

Aplicando o Lema 4.1.2, existe uma constante $C(\epsilon) \rightarrow 0$ quando $\epsilon \rightarrow 0$ tal que

$$
\left\|e^{-A^{\epsilon} t} u^{\epsilon}-e^{-A^{0} t} u^{\epsilon}\right\|_{H^{1}(\Omega)} \leq C(\epsilon) t^{-\left(\frac{1+\alpha}{2}\right)}\left\|u^{\epsilon}\right\|_{H^{-\alpha}(\Omega)}, \quad \forall t \in(0, T] .
$$

Pelo Teorema 4.2.1 $\mathcal{A}_{\epsilon}, 0<\epsilon \leq \epsilon_{0}$, é uniformemente limitado em $H^{1}(\Omega) \cap L^{\infty}(\Omega)$. Portanto, $u^{\epsilon} \in \mathcal{A}_{\epsilon}$ também é uniformemente limitada em $H^{1}(\Omega) \cap L^{\infty}(\Omega)$. Além disso, como $H^{1}(\Omega) \hookrightarrow H^{-\alpha}(\Omega)$ (vide o Teorema 2.2.18), temos que existe uma constante $C_{1}(\epsilon) \rightarrow 0$, quando $\epsilon \rightarrow 0$, tal que

$$
N_{1} \leq C_{1}(\epsilon) t^{-\left(\frac{1+\alpha}{2}\right)} .
$$

\section{Caso $\mathrm{N}_{2}$}


Somando e subtraindo os termos

- $e^{-A^{\epsilon}(t-s)} h^{0}\left(T^{\epsilon}(s) u^{\epsilon}\right)$;

- $e^{-A^{0}(t-s)} h^{0}\left(T^{\epsilon}(s) u^{\epsilon}\right)$

dividimos $N_{2}$ em 3 parcelas $N_{2} \leq I_{1}+I_{2}+I_{3}$, onde

1. $I_{1}=\left\|e^{-A^{\epsilon}(t-s)} h^{\epsilon}\left(T^{\epsilon}(s) u^{\epsilon}\right)-e^{-A^{\epsilon}(t-s)} h^{0}\left(T^{\epsilon}(s) u^{\epsilon}\right)\right\|_{H^{1}(\Omega)}$;

2. $I_{2}=\left\|e^{-A^{\epsilon}(t-s)} h^{0}\left(T^{\epsilon}(s) u^{\epsilon}\right)-e^{-A^{0}(t-s)} h^{0}\left(T^{\epsilon}(s) u^{\epsilon}\right)\right\|_{H^{1}(\Omega)}$;

3. $I_{3}=\left\|e^{-A^{0}(t-s)} h^{0}\left(T^{\epsilon}(s) u^{\epsilon}\right)-e^{-A^{0}(t-s)} h^{0}\left(T^{0}(s) u^{\epsilon}\right)\right\|_{H^{1}(\Omega)}$.

Primeiro, vamos fazer uma observação: pela invariância de $\mathcal{A}_{\epsilon}$ por $T^{\epsilon}(t)$ temos que, se $u^{\epsilon} \in \mathcal{A}_{\epsilon}$, então $T^{\epsilon}(t) u^{\epsilon} \in \mathcal{A}_{\epsilon}$, e ainda, pelo Teorema 4.2.1 $\mathcal{A}_{\epsilon}$ é uniformemente limitado em $H^{1}(\Omega) \cap L^{\infty}(\Omega)$, portanto $T^{\epsilon}(t) u^{\epsilon} \in \mathcal{A}_{\epsilon}$ também é uniformemente limitado em $H^{1}(\Omega) \cap L^{\infty}(\Omega)$. Logo, existe uma constante $K>0$ independente de $\epsilon$ tal que

$$
\left\|T^{\epsilon}(s) u^{\epsilon}\right\|_{H^{1}(\Omega) \cap L^{\infty}(\Omega)} \leq K, \quad \forall s \in[0, T] \quad \text { e } \quad 0 \leq \epsilon \leq \epsilon_{0} .
$$

Agora, vamos estimar $I_{1}$ : usando a estimativa do semigrupo (3.7) para $t \in(0, T]$ e $s \in[0, t]$, temos

$$
I_{1} \leq M_{2} e^{-\beta(t-s)}(t-s)^{-\left(\frac{1+\alpha}{2}\right)}\left\|h^{\epsilon}\left(T^{\epsilon}(s) u^{\epsilon}\right)-h^{0}\left(T^{\epsilon}(s) u^{\epsilon}\right)\right\|_{H^{-\alpha}(\Omega)} .
$$

Usando (4.3) e o Lema 4.1.1, existe $M(T, K, \epsilon) \rightarrow 0$, quando $\epsilon \rightarrow 0$, tal que

$$
\left\|h^{\epsilon}\left(T^{\epsilon}(s) u^{\epsilon}\right)-h^{0}\left(T^{\epsilon}(s) u^{\epsilon}\right)\right\|_{H^{-\alpha}(\Omega)} \leq M(T, K, \epsilon) .
$$

Portanto, para $t \in(0, T]$ e $s \in[0, t]$,

$$
I_{1} \leq M_{2} M(T, K, \epsilon) e^{-\beta(t-s)}(t-s)^{-\left(\frac{1+\alpha}{2}\right)} \leq M_{2} M(T, K, \epsilon)(t-s)^{-\left(\frac{1+\alpha}{2}\right)} .
$$

Vamos estimar $I_{2}$ : usando a convergência do semigrupo linear (Lema 4.1.2), existe $C(\epsilon) \rightarrow 0$, quando $\epsilon \rightarrow 0$, tal que para $t \in(0, T]$ e $s \in[0, t]$, temos

$$
I_{2} \leq\left\|\left[e^{-A^{\epsilon}(t-s)}-e^{-A^{0}(t-s)}\right] h^{0}\left(T^{\epsilon}(s) u^{\epsilon}\right)\right\|_{H^{1}(\Omega)} \leq C(\epsilon)(t-s)^{-\left(\frac{1+\alpha}{2}\right)}\left\|h^{0}\left(T^{\epsilon}(s) u^{\epsilon}\right)\right\|_{H^{-\alpha}(\Omega)} .
$$

Usando (4.3) e o Lema 3.3.1, temos que existe $C(T, K)>0$, independente de $\epsilon$, tal que

$$
\left\|h^{0}\left(T^{\epsilon}(s) u^{\epsilon}\right)\right\|_{H^{-\alpha}(\Omega)} \leq C(T, K), \quad \forall s \in[0, T] .
$$

Portanto, para $t \in(0, T]$ e $s \in[0, t]$, temos

$$
I_{2} \leq C(\epsilon) C(T, K)(t-s)^{-\left(\frac{1+\alpha}{2}\right)} .
$$

Vamos estimar $I_{3}$ : usando a estimativa do semigrupo (3.7), para $t \in(0, T]$ e $s \in[0, t]$, temos

$$
\begin{aligned}
I_{3} & \leq\left\|e^{-A^{0}(t-s)}\left[h^{0}\left(T^{\epsilon}(s) u^{\epsilon}\right)-h^{0}\left(T^{0}(s) u^{\epsilon}\right)\right]\right\|_{H^{1}(\Omega)} \\
& \leq M_{2} e^{-\beta(t-s)}(t-s)^{-\left(\frac{1+\alpha}{2}\right)}\left\|h^{0}\left(T^{\epsilon}(s) u^{\epsilon}\right)-h^{0}\left(T^{0}(s) u^{\epsilon}\right)\right\|_{H^{-\alpha}(\Omega)} .
\end{aligned}
$$

Agora por (4.3) e usando o Lema 3.3.2, obtemos que existe uma constante $L(T, K)>0$ independente de $\epsilon$ tal que

$$
\left\|h^{0}\left(T^{\epsilon}(s) u^{\epsilon}\right)-h^{0}\left(T^{0}(s) u^{\epsilon}\right)\right\|_{H^{-\alpha}(\Omega)} \leq L(T, K)\left\|T^{\epsilon}(s) u^{\epsilon}-T^{0}(s) u^{\epsilon}\right\|_{H^{1}(\Omega)} .
$$


Portanto, para $t \in(0, T]$ e $s \in[0, t]$, temos

$$
I_{3} \leq M_{2} L(T, K)(t-s)^{-\left(\frac{1+\alpha}{2}\right)}\left\|T^{\epsilon}(s) u^{\epsilon}-T^{0}(s) u^{\epsilon}\right\|_{H^{1}(\Omega)} .
$$

Procedendo de forma análoga ao Teorema 4.1.3, para $t \in(0, T]$, existem constantes positivas $C_{2}(K, \epsilon)$ e $C_{3}(T, K)$, com $C_{2}(K, \epsilon) \rightarrow 0$, quando $\epsilon \rightarrow 0$, tais que

$$
\left\|T^{\epsilon}(s) u^{\epsilon}-T^{0}(s) u^{\epsilon}\right\|_{H^{1}(\Omega)} \leq C_{2}(K, \epsilon) t^{-\left(\frac{1+\alpha}{2}\right)}+C_{3}(T, K) \int_{0}^{t}(t-s)^{-\left(\frac{1+\alpha}{2}\right)}\left\|T^{\epsilon}(s) u^{\epsilon}-T^{0}(s) u^{\epsilon}\right\|_{H^{1}(\Omega)} \mathrm{d} s .
$$

Aplicando a desigualdade de Gronwall (Lema 2.5.5), temos que existe uma constante positiva $C(T, K, \epsilon) \rightarrow 0$, quando $\epsilon \rightarrow 0$, tal que

$$
\left\|T^{\epsilon}(t) u^{\epsilon}-T^{0}(t) u^{\epsilon}\right\|_{H^{1}(\Omega)} \leq C(T, K, \epsilon) t^{-\left(\frac{1+\alpha}{2}\right)}, \quad \forall t \in(0, T] .
$$

Teorema 4.2.3 Suponha que valem as hipóteses (H1), (H2), (H3) e (P). Então, a familia de atratores globais $\left\{\mathcal{A}_{\epsilon}\right\}_{\epsilon \in\left[0, \epsilon_{0}\right]}$ de (1.1), $0<\epsilon \leq \epsilon_{0}$, e (1.4), $\epsilon=0$, é semicontinua superiormente em $\epsilon=0$, isto é,

$$
d_{H^{1}(\Omega)}\left(\mathcal{A}_{\epsilon}, \mathcal{A}_{0}\right)=\sup _{u^{\epsilon} \in \mathcal{A}_{\epsilon}} d\left(u^{\epsilon}, \mathcal{A}_{0}\right)=\sup _{u^{\epsilon} \in \mathcal{A}_{\epsilon}} \inf _{u^{0} \in \mathcal{A}_{0}}\left\|u^{\epsilon}-u^{0}\right\|_{H^{1}(\Omega)} \rightarrow 0, \quad \text { quando } \quad \epsilon \rightarrow 0 .
$$

\section{Demonstração:}

Pelo Teorema 4.2.1, $\mathcal{A}_{0}$ atrai $\bigcup_{0<\epsilon \leq \epsilon_{0}} \mathcal{A}_{\epsilon}$. Logo, dado $\eta>0$, existe $\tau_{*}=\tau_{*}(\eta)>0$ independente de $\epsilon$ tal que

$$
d\left(T^{0}\left(\tau_{*}\right) u^{\epsilon}, \mathcal{A}_{0}\right)=\inf _{u^{0} \in \mathcal{A}_{0}}\left\|T^{0}\left(\tau_{*}\right) u^{\epsilon}-u^{0}\right\|_{H^{1}(\Omega)} \leq \frac{\eta}{2}, \quad \forall u^{\epsilon} \in \mathcal{A}_{\epsilon} \quad \text { e } \quad \epsilon \in\left(0, \epsilon_{0}\right] .
$$

Como $\mathcal{A}_{\epsilon}$ é invariante por $T^{\epsilon}(t), \epsilon \in\left(0, \epsilon_{0}\right]$, então, $T^{\epsilon}(t) \mathcal{A}_{\epsilon}=\mathcal{A}_{\epsilon}, \forall t \geq 0$. Assim, dado $v^{\epsilon} \in \mathcal{A}_{\epsilon}$, existe $u^{\epsilon} \in \mathcal{A}_{\epsilon}$ tal que $T^{\epsilon}\left(\tau_{*}\right) u^{\epsilon}=v^{\epsilon}$. Logo,

$$
\begin{aligned}
d\left(v^{\epsilon}, \mathcal{A}_{0}\right) & =\inf _{u^{0} \in \mathcal{A}_{0}}\left\|v^{\epsilon}-u^{0}\right\|_{H^{1}(\Omega)} \leq \inf _{u^{0} \in \mathcal{A}_{0}}\left\{\left\|v^{\epsilon}-T^{0}\left(\tau_{*}\right) u^{\epsilon}\right\|_{H^{1}(\Omega)}+\left\|T^{0}\left(\tau_{*}\right) u^{\epsilon}-u^{0}\right\|_{H^{1}(\Omega)}\right\} \\
& =\left\|v^{\epsilon}-T^{0}\left(\tau_{*}\right) u^{\epsilon}\right\|_{H^{1}(\Omega)}+d\left(T^{0}\left(\tau_{*}\right) u^{\epsilon}, \mathcal{A}_{0}\right) \\
& =\left\|T^{\epsilon}\left(\tau_{*}\right) u^{\epsilon}-T^{0}\left(\tau_{*}\right) u^{\epsilon}\right\|_{H^{1}(\Omega)}+d\left(T^{0}\left(\tau_{*}\right) u^{\epsilon}, \mathcal{A}_{0}\right) \\
& \leq\left\|T^{\epsilon}\left(\tau_{*}\right) u^{\epsilon}-T^{0}\left(\tau_{*}\right) u^{\epsilon}\right\|_{H^{1}(\Omega)}+\frac{\eta}{2} .
\end{aligned}
$$

Agora, como $u^{\epsilon} \in \mathcal{A}_{\epsilon}, 0<\epsilon \leq \epsilon_{0}$, então usando o Lema 4.2.2, obtemos

$$
\left\|T^{\epsilon}\left(\tau_{*}\right) u^{\epsilon}-T^{0}\left(\tau_{*}\right) u^{\epsilon}\right\|_{H^{1}(\Omega)} \leq \frac{\eta}{2}
$$

para $\epsilon$ suficientemente pequeno. Portanto

$$
d\left(v^{\epsilon}, \mathcal{A}_{0}\right) \leq \eta, \quad \forall v^{\epsilon} \in \mathcal{A}_{\epsilon} .
$$

Assim, para $\epsilon$ suficientemente pequeno,

$$
d_{H^{1}(\Omega)}\left(\mathcal{A}_{\epsilon}, \mathcal{A}_{0}\right)=\sup _{v^{\epsilon} \in \mathcal{A}_{\epsilon}} d\left(v^{\epsilon}, \mathcal{A}_{0}\right) \leq \eta
$$




\subsection{Semicontinuidade superior dos equilíbrios}

Na Seção 4.2, supondo as hipóteses (H1), (H2), (H3) e (P), mostramos a existência e semicontinuidade superior da família de atratores $\left\{\mathcal{A}_{\epsilon}\right\}_{\epsilon \in\left[0, \epsilon_{0}\right]}$ de $(1.1)$ e (1.4) em $\epsilon=0$. Um primeiro passo para estudarmos a semicontinuidade inferior dessa família de atratores é estudar os elementos mais simples dos atratores, as soluções de equilíbrios. As soluções de equilíbrios de (1.1) e (1.4) são aquelas que independem do tempo, isto é, para cada $\epsilon \in\left[0, \epsilon_{0}\right]$,

$$
T^{\epsilon}(t) u_{\epsilon}=u_{\epsilon}, \quad \forall t \geq 0
$$

ou ainda, são as soluções dos respectivos problemas elípticos

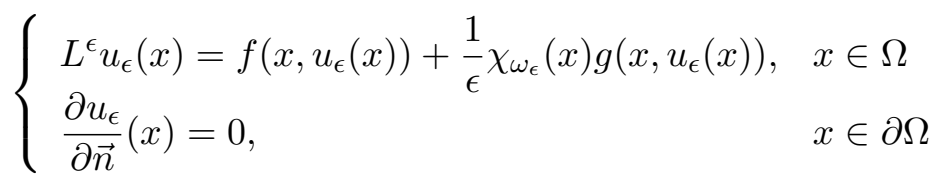

onde $L^{\epsilon}$ é dado por (1.2).

$$
\begin{cases}L^{0} u_{0}(x)=f\left(x, u_{0}(x)\right), & x \in \Omega \\ \frac{\partial u_{0}}{\partial \vec{n}}(x)+V_{0}(x) u_{0}(x)=g\left(x, u_{0}(x)\right), & x \in \partial \Omega\end{cases}
$$

onde $L^{0}$ é dado por (1.5).

Nesta seção, vamos provar a semicontinuidade superior do conjunto de equilíbrios de (1.1) e (1.4) em $\epsilon=0$.

\subsubsection{Formulação abstrata dos problemas elípticos}

Inicialmente, vamos escrever os problemas elípticos (4.4) e (4.5) na forma abstrata. Usando as notações e procedendo de maneira análoga a Seção 3.1, podemos reescrever (4.4) e (4.5) da seguinte forma:

$$
A^{\epsilon} u_{\epsilon}=h^{\epsilon}\left(u_{\epsilon}\right), \quad 0 \leq \epsilon \leq \epsilon_{0}
$$

onde $h^{0}=F+G^{0}$ e $h^{\epsilon}=F+G^{\epsilon}, 0<\epsilon \leq \epsilon_{0}$, com $F, G^{0}$ e $G^{\epsilon}$ dadas por (3.8), (3.9) e (3.10), respectivamente.

Denotemos por $\mathcal{E}_{\epsilon}, 0 \leq \epsilon \leq \epsilon_{0}$, o conjunto das soluções de (4.6), ou seja, o conjunto de pontos de equilíbrios de (1.1) e (1.4),

$$
\mathcal{E}_{\epsilon}=\left\{u_{\epsilon} \in H^{1}(\Omega): \quad A^{\epsilon} u_{\epsilon}-h^{\epsilon}\left(u_{\epsilon}\right)=0\right\}, \quad 0 \leq \epsilon \leq \epsilon_{0} .
$$

\subsubsection{Semicontinuidade superior dos equilíbrios}

Agora, vamos provar a semicontinuidade superior da família de equilíbrios $\left\{\mathcal{E}_{\epsilon}\right\}_{\epsilon \in\left[0, \epsilon_{0}\right]}$ em $\epsilon=0$. Para tanto, usaremos o fato que $\mathcal{E}_{\epsilon} \subset \mathcal{A}_{\epsilon}$ e que a família de atratores $\left\{\mathcal{A}_{\epsilon}\right\}_{\epsilon \in\left[0, \epsilon_{0}\right]}$ é semicontínua superiormente em $\epsilon=0$.

Teorema 4.3.1 Suponha que valem as hipóteses (H1), (H2), (H3) e (P). Então, a familia de equilíbrios $\left\{\mathcal{E}_{\epsilon}\right\}_{\epsilon \in\left[0, \epsilon_{0}\right]}$ de (1.1), $0<\epsilon \leq \epsilon_{0}$, e (1.4), $\epsilon=0$, é semicontínua superiormente em $\epsilon=0$.

\section{Demonstração:}

Para provarmos a semicontinuidade superior em $H^{1}(\Omega)$ da família $\left\{\mathcal{E}_{\epsilon}\right\}_{\epsilon \in\left[0, \epsilon_{0}\right]}$ em $\epsilon=0$, pelo Lema 2.6.11, é suficiente mostrarmos que para qualquer sequência $\epsilon \rightarrow 0$ e para qualquer sequência de equilíbrios $u_{\epsilon} \in \mathcal{E}_{\epsilon}$, existe uma subsequência convergente com limite pertencendo a $\mathcal{E}_{0}$.

Como $u_{\epsilon} \in \mathcal{E}_{\epsilon} \subset \mathcal{A}_{\epsilon}$, e pelo Teorema 4.2.3, temos a semicontinuidade superior da família de atratores, então usando o Lema 2.6.11, podemos extrair uma subsequência, que denotaremos por 
$\left\{u_{\epsilon_{k}}\right\}_{k \in \mathbb{N}}$, e obter $u_{0} \in \mathcal{A}_{0}$ tal que

$$
\left\|u_{\epsilon_{k}}-u_{0}\right\|_{H^{1}(\Omega)} \rightarrow 0, \quad \text { quando } \quad k \rightarrow \infty .
$$

Agora, precisamos mostrar que $u_{0} \in \mathcal{E}_{0}$, isto é, $T^{0}(t) u_{0}=u_{0}, \forall t \geq 0$.

Primeiramente, observe que para todo $t>0$,

$$
\left\|u_{\epsilon_{k}}-T^{0}(t) u_{0}\right\|_{H^{1}(\Omega)} \leq\left\|u_{\epsilon_{k}}-u_{0}\right\|_{H^{1}(\Omega)}+\left\|u_{0}-T^{0}(t) u_{0}\right\|_{H^{1}(\Omega)} \rightarrow\left\|u_{0}-T^{0}(t) u_{0}\right\|_{H^{1}(\Omega)},
$$

quando $k \rightarrow \infty$.

Por outro lado, para um $\tau_{*}>0$ fixo e para todo $t \in\left(0, \tau_{*}\right)$, temos

$$
\begin{aligned}
\left\|u_{\epsilon_{k}}-T^{0}(t) u_{0}\right\|_{H^{1}(\Omega)} & =\left\|T^{\epsilon_{k}}(t) u_{\epsilon_{k}}-T^{0}(t) u_{0}\right\|_{H^{1}(\Omega)} \\
& \leq\left\|T^{\epsilon_{k}}(t) u_{\epsilon_{k}}-T^{0}(t) u_{\epsilon_{k}}\right\|_{H^{1}(\Omega)}+\left\|T^{0}(t) u_{\epsilon_{k}}-T^{0}(t) u_{0}\right\|_{H^{1}(\Omega)} .
\end{aligned}
$$

Pelo Lema 4.2.2, temos que

$$
\left\|T^{\epsilon_{k}}(t) u_{\epsilon_{k}}-T^{0}(t) u_{\epsilon_{k}}\right\|_{H^{1}(\Omega)} \rightarrow 0, \quad \text { quando } \quad k \rightarrow \infty .
$$

E ainda, como $T^{0}(t) \in \mathcal{L}\left(H^{1}(\Omega)\right)$ e $u_{\epsilon_{k}} \rightarrow u_{0}$ em $H^{1}(\Omega)$, quando $k \rightarrow \infty$, então

$$
\left\|T^{0}(t) u_{\epsilon_{k}}-T^{0}(t) u_{0}\right\|_{H^{1}(\Omega)} \rightarrow 0, \quad \text { quando } \quad k \rightarrow \infty .
$$

Portanto, para todo $t \in\left(0, \tau_{*}\right)$, obtemos

$$
\left\|u_{\epsilon_{k}}-T^{0}(t) u_{0}\right\|_{H^{1}(\Omega)} \rightarrow 0, \quad \text { quando } \quad k \rightarrow \infty .
$$

Logo, para cada $t>0, u_{0}=T^{0}(t) u_{0}$, o que implica que $u_{0} \in \mathcal{E}_{0}$.

\subsection{Considerações finais}

Neste trabalho, procuramos explorar alguns resultados de Jiménes-Casas e Rodríguez-Bernal $(2009,2011)$ tendo como objetivo analisar o comportamento assintótico do problema parabólico não linear concentrado (1.1). Mostramos que o problema limite de (1.1) é dado pelo problema parabólico não linear (1.4). Esse resultado de convergência pode ser visto como uma ferramenta para transferir informações do interior para a fronteira. Mostramos também a existência e semicontinuidade superior da família de atratores e, com isso, concluímos a semicontinuidade da família de equilíbrios.

Com base nos resultados obtidos aqui, atualmente, estamos estudando a semicontinuidade inferior da família de equilíbrios. Os próximos passos serão obter a semicontinuidade inferior da família de atratores. 


\section{Apêndice A}

\section{Outra abordagem para provar a condição de Lipschitz}

A existência e unicidade de equações diferenciais estão condicionadas, no geral, as funções Lipschitzianas, contudo, nem sempre é possível concluir essa condição para qualquer espaço. Discutiremos aqui outras formas de obter essa condição de Lipschitz para as não linearidades abstratas.

Note que até então, com a hipótese (H1), na Seção 3.2, só foi possível mostrar que as aplicações abstratas são Lipschitz de $H^{1}(\Omega)$ em $H^{-1}(\Omega)$, contudo, podemos exigir mais das funções, como por exemplo, alterar os coeficientes $\sigma_{f}$ e $\sigma_{g}$ para também concluir de $H^{1}(\Omega)$ em $H^{-\alpha}(\Omega)$, com $\frac{1}{2}<\alpha \leq 1$. Considere a nova hipótese $\left(\mathrm{H} 1^{*}\right)$

\section{$(\mathrm{H} 1 *)$ - Nova condição de crescimento.}

Suponha que $f, g: \bar{\Omega} \times \mathbb{R} \rightarrow \mathbb{R}$ são funções uniformemente contínuas, $f(x, \cdot), g(x, \cdot): \mathbb{R} \rightarrow \mathbb{R}$ são localmente Lipschitzianas uniformemente em $x \in \Omega$ e $x \in \bar{\Omega}$, respectivamente, e satisfazem as seguintes condições de crescimento:

i. Se $n>2$, existe $c>0$ tal que

$$
|j(x, u)-j(x, v)| \leq c|u-v|\left(|u|^{\sigma_{j}}+|v|^{\sigma_{j}}+1\right),
$$

onde $j=f$ ou $j=g$ com expoentes $\sigma_{f}$ e $\sigma_{g}$, respectivamente, tais que

$$
\sigma_{f} \leq \frac{3}{n-2} \quad \text { e } \quad \sigma_{g} \leq \frac{1}{n-2}
$$

ii. Se $n=2$, para qualquer $\eta>0$ existe $c_{\eta}>0$ tal que

$$
|j(x, u)-j(x, v)| \leq c_{\eta}|u-v|\left(e^{\eta|u|^{2}}+e^{\eta|v|^{2}}\right),
$$

onde $j=f$ ou $j=g$.

Cabe ressaltar que não encontramos esta condição para $n \geq 2$ de forma explícita nos artigos estudados, no quais abordam problemas de existência e unicidade de soluções. Apenas em Carvalho et al. (1997) encontramos um estudo para $n \leq 3$ (Lema 3.4) e em Aragao e Bezerra (2017) um caso $n=3$.

Proposição A.0.1 Suponha que $f$ satisfaz $\left(H 1^{*}\right) e \frac{1}{2}<\alpha \leq 1$. Então, a aplicação

$$
F: H^{1}(\Omega) \rightarrow H^{-\alpha}(\Omega)
$$

dada por (3.8) é localmente Lipschitziana.

\section{Demonstração:}


Sejam $u, v \in H^{1}(\Omega) \operatorname{com}\|u\|_{H^{1}(\Omega)},\|v\|_{H^{1}(\Omega)} \leq R$, para algum $R>0$. Por definição,

$$
\|F(u)-F(v)\|_{H^{-\alpha}(\Omega)}=\sup _{\substack{\phi \in H^{\alpha}(\Omega) \\\|\phi\|_{H^{\alpha}(\Omega)}=1}}|\langle F(u)-F(v), \phi\rangle|,
$$

com

$$
|\langle F(u)-F(v), \phi\rangle| \leq \int_{\Omega}|f(x, u(x))-f(x, v(x))||\phi(x)| \mathrm{d} x, \quad \forall \phi \in H^{\alpha}(\Omega) .
$$

Para $n=2$ :

Como vale a imersão $H^{\alpha}(\Omega) \hookrightarrow L^{4}(\Omega)$, com $\frac{1}{2}<\alpha \leq 1$, a demonstração segue exatamente igual a Proposição 3.2.2.

Assim, temos que existe uma constante $K_{7}=K_{7}(\eta,|\Omega|, R)>0$ tal que

$$
\|F(u)-F(v)\|_{H^{-\alpha}(\Omega)} \leq K_{7}\|u-v\|_{H^{1}(\Omega)},
$$

$\operatorname{com} \frac{1}{2}<\alpha \leq 1$.

Para $n>2$ :

Por $\left(\mathrm{H} 1^{*}\right)$,

$$
|\langle F(u)-F(v), \phi\rangle| \leq c \int_{\Omega}|u(x)-v(x)|\left(|u(x)|^{\sigma_{f}}+|v(x)|^{\sigma_{f}}+1\right)|\phi(x)| \mathrm{d} x .
$$

Utilizando a desigualdade de Hölder com $\frac{1}{q}+\frac{1}{r}+\frac{1}{p}=1$, e a desigualdade de Minkwoski, temos

$$
|\langle F(u)-F(v), \phi\rangle| \leq \tilde{c}\|u-v\|_{L^{r}(\Omega)}\left(\left\|u^{\sigma_{f}}\right\|_{L^{p}(\Omega)}+\left\|v^{\sigma_{f}}\right\|_{L^{p}(\Omega)}+1\right)\|\phi\|_{L^{q}(\Omega)},
$$

onde $\tilde{c}=\tilde{c}(|\Omega|)>0$, ou ainda,

$$
\begin{aligned}
|\langle F(u)-F(v), \phi\rangle| & \leq \tilde{c}\|u-v\|_{L^{r}(\Omega)}\left(\|u\|_{L^{\sigma_{f}}(\Omega)}^{\sigma_{f}}+\|v\|_{L^{\sigma_{f}}(\Omega)}^{\sigma_{f}}+1\right)\|\phi\|_{L^{q}(\Omega)} \\
& \leq \tilde{c}\|u-v\|_{H^{1}(\Omega)}\left(\|u\|_{H^{1}(\Omega)}^{\sigma_{f}}+\|v\|_{H^{1}(\Omega)}^{\sigma^{1}}+1\right)\|\phi\|_{H^{\alpha}(\Omega)},
\end{aligned}
$$

onde precisamos que

$$
H^{\alpha}(\Omega) \hookrightarrow L^{q}(\Omega), \quad H^{1}(\Omega) \hookrightarrow L^{r}(\Omega) \quad \text { e } \quad H^{1}(\Omega) \hookrightarrow L^{p \sigma_{f}}(\Omega) .
$$

Pelo Teorema 2.2.18, devemos ter:

- $1<r \leq \frac{2 n}{n-2}$;

- $1<q \leq \frac{2 n}{n-1}$;

- $p \sigma_{f} \leq \frac{2 n}{n-2}$.

Assim,

$$
\frac{1}{r}+\frac{1}{q} \geq \frac{n-2}{2 n}+\frac{n-1}{2 n}=\frac{2 n-3}{2 n} .
$$

Por outro lado,

$$
\frac{1}{r}+\frac{1}{q}=1-\frac{1}{p} \geq \frac{2 n-3}{2 n} .
$$

Portanto, se $H^{\alpha}(\Omega) \hookrightarrow L^{q}(\Omega)$ e $H^{1}(\Omega) \hookrightarrow L^{r}(\Omega)$, segue que $p \geq \frac{2 n}{3}$. 
Por fim, basta que $\int_{\Omega}|u(x)|^{p_{f}} \mathrm{~d} x<\infty$, mas isso decorre se tomarmos $p$ tal que $H^{1}(\Omega) \hookrightarrow$ $L^{p \sigma_{f}}(\Omega)$, isto é,

$$
p \sigma_{f} \leq \frac{2 n}{n-2} .
$$

Porém, pela $\left(\mathrm{H} 1^{*}\right)$ e das imersões acima,

$$
\sigma_{f} \leq \frac{3}{n-2} \quad \text { e } \quad p \geq \frac{2 n}{3} .
$$
$v$.

Logo, basta escolher, por exemplo $p=\frac{2 n}{3}$ e segue que $\int_{\Omega}|u(x)|^{p \sigma_{f}} \mathrm{~d} x<\infty$, analogamente para

Sob essas condições, tomando o supremo com $\|\phi\|_{H^{\alpha}(\Omega)}=1$, temos que existe uma constante $K_{8}=K_{8}(|\Omega|, R)>0$ tal que

$$
\|F(u)-F(v)\|_{H^{-\alpha}(\Omega)} \leq K_{8}\|u-v\|_{H^{1}(\Omega)},
$$

$\operatorname{com} \frac{1}{2}<\alpha \leq 1$.

Proposição A.0.2 Suponha que g satisfaz $\left(H 1^{*}\right) e \frac{1}{2}<\alpha \leq 1$. Então, para cada $\epsilon \in\left[0, \epsilon_{0}\right]$, a aplicação

$$
G^{\epsilon}: H^{1}(\Omega) \rightarrow H^{-\alpha}(\Omega),
$$

dada por (3.9) e (3.10) é localmente Lipschitziana, uniformemente em $\epsilon$.

\section{Demonstração:}

Sejam $u, v \in H^{1}(\Omega)$ com $\|u\|_{H^{1}(\Omega)},\|v\|_{H^{1}(\Omega)} \leq R$, para algum $R>0$. Por definição, para cada $\epsilon \in\left[0, \epsilon_{0}\right]$

$$
\left\|G^{\epsilon}(u)-G^{\epsilon}(v)\right\|_{H^{-\alpha}(\Omega)}=\sup _{\substack{\phi \in H^{\alpha}(\Omega) \\\|\phi\|_{H^{\alpha}(\Omega)}=1}}\left|\left\langle G^{\epsilon}(u)-G^{\epsilon}(v), \phi\right\rangle\right| .
$$

Para cada $\phi \in H^{\alpha}(\Omega)$ e $0<\epsilon \leq \epsilon_{0}$, vamos estimar $\mid\left\langle G^{\epsilon}(u)-G^{\epsilon}(v), \phi\right\rangle$ :

$$
\left|\left\langle G^{\epsilon}(u)-G^{\epsilon}(v), \phi\right\rangle\right| \leq \frac{1}{\epsilon} \int_{\omega_{\epsilon}}|[g(x, u(x))-g(x, v(x))] \phi(x)| \mathrm{d} x .
$$

Para $n=2$ :

Considere $H_{p}^{\alpha}(\Omega)=H^{\alpha}(\Omega), \frac{1}{2}<\alpha \leq 1$, e $n=2$, sob essas condições o Lema 2.7.1 valeu para algum $L^{q}(\Omega)$, com $1<q \leq 4$, portanto, esse caso segue análago a demonstração da Proposição 3.2.3. Assim, existe uma constante $K_{9}=K_{9}(\eta,|\partial \Omega|, R)>0$, independente de $\epsilon$, tal que

$$
\left\|G^{\epsilon}(u)-G^{\epsilon}(v)\right\|_{H^{-\alpha}(\Omega)} \leq K_{9}\|u-v\|_{H^{1}(\Omega)},
$$

$\operatorname{com} \frac{1}{2}<\alpha \leq 1$.

Para $n>2$ :

Por $\left(\mathrm{H} 1^{*}\right)$,

$$
\left|\left\langle G^{\epsilon}(u)-G^{\epsilon}(v), \phi\right\rangle\right| \leq c \frac{1}{\epsilon} \int_{\omega_{\epsilon}}|u(x)-v(x)|\left(|u(x)|^{\sigma_{g}}+|v(x)|^{\sigma_{g}}+1\right)|\phi(x)| \mathrm{d} x .
$$


Utilizando a desigualdade de Hölder, com $\frac{1}{q}+\frac{1}{r}+\frac{1}{p}=1$, temos

$$
\begin{array}{r}
\left|\left\langle G^{\epsilon}(u)-G^{\epsilon}(v), \phi\right\rangle\right| \leq c\left(\frac{1}{\epsilon} \int_{\omega_{\epsilon}}|u(x)-v(x)|^{r} \mathrm{~d} x\right)^{\frac{1}{r}}\left(\frac{1}{\epsilon} \int_{\omega_{\epsilon}}|\phi(x)|^{q} \mathrm{~d} x\right)^{\frac{1}{q}} \\
\left(\frac{1}{\epsilon} \int_{\omega_{\epsilon}}\left(|u(x)|^{\sigma_{g}}+|v(x)|^{\sigma_{g}}+1\right)^{p} \mathrm{~d} x\right)^{\frac{1}{p}} .
\end{array}
$$

Usando também a desigualdade de Minkwoski, obtemos

$$
\begin{array}{r}
\left|\left\langle G^{\epsilon}(u)-G^{\epsilon}(v), \phi\right\rangle\right| \leq c\left(\frac{1}{\epsilon}\right)^{\frac{1}{r}}\|u-v\|_{L^{r}\left(\omega_{\epsilon}\right)}\left(\frac{1}{\epsilon}\right)^{\frac{1}{q}}\|\phi\|_{L^{q}\left(\omega_{\epsilon}\right)} \\
{\left[\left(\frac{1}{\epsilon}\right)^{\frac{1}{p}}\left\|u^{\sigma_{g}}\right\|_{L^{p}\left(\omega_{\epsilon}\right)}+\left(\frac{1}{\epsilon}\right)^{\frac{1}{p}}\left\|v^{\sigma_{g}}\right\|_{L^{p}\left(\omega_{\epsilon}\right)}+\left(\frac{\left|\omega_{\epsilon}\right|}{\epsilon}\right)^{\frac{1}{p}}\right] .}
\end{array}
$$

Primeiro, note que $\left(\frac{1}{\epsilon}\right)^{\frac{1}{p}}\left\|u^{\sigma_{g}}\right\|_{L^{p}\left(\omega_{\epsilon}\right)}=\left[\left(\frac{1}{\epsilon}\right)^{\frac{1}{p \sigma_{g}}}\|u\|_{L^{p \sigma_{g}\left(\omega_{\epsilon}\right)}}\right]^{\sigma_{g}}$, o mesmo vale para $v$.

Assim, considerando que $u \in H^{1}(\Omega)$ e $\phi \in H^{\alpha}(\Omega), \frac{1}{2}<\alpha \leq 1$, podemos usar o Lema 2.7.1 para algum $L^{r}(\Omega)$, respectivamente para $q$ e $p \sigma_{g}$, onde basta tomar os expoentes:

- $1<r \leq \frac{2(n-1)}{n-2}$;

- $1<q \leq 2$;

- $1<p \sigma_{g} \leq \frac{2(n-1)}{n-2}$.

Assim,

$$
\frac{1}{r}+\frac{1}{q} \geq \frac{n-2}{2(n-1)}+\frac{1}{2}=\frac{2 n-3}{2(n-1)} .
$$

Por outro lado,

$$
\frac{1}{r}+\frac{1}{q}=1-\frac{1}{p} \geq \frac{2 n-3}{2(n-1)}
$$

Portanto, se vale o Lema 2.7.1, para $r, q$ nos intervalos acima, respectivamente, então $p \geq$ $2(n-1)$.

Já por $\left(\mathrm{H} 1^{*}\right), \sigma_{g} \leq \frac{1}{n-2}$, portanto, escolhendo, por exemplo, $p=2(n-1)$, segue que

$$
p \sigma_{g} \leq \frac{2(n-1)}{n-2} .
$$

Assim, escolhendo $r, q, p \sigma_{g}$ desta forma, a demonstração segue análogo a Proposição 3.2.3. Logo, existe uma constante $K_{10}=K_{10}(|\partial \Omega|, R)>0$, independente de $\epsilon$, tal que

$$
\left\|G^{\epsilon}(u)-G^{\epsilon}(v)\right\|_{H^{-\alpha}(\Omega)} \leq K_{10}\|u-v\|_{H^{1}(\Omega)},
$$

$\operatorname{com} \frac{1}{2}<\alpha \leq 1$.

Para $\epsilon=0$ a demonstração e as contas são exatamente iguais, pois note que as restrições sobre os expoentes da desigualdade de Hölder no Lema 2.7.1 e para que o traço seja um operador contínuo de $H^{\alpha}(\Omega) \rightarrow L^{r}(\partial \Omega)$, para algum $r$ devem ser as mesmas, isto é,

$$
\alpha-\frac{n}{2} \geq-\frac{n-1}{r}
$$


e $\frac{1}{2}<\alpha \leq 1$. Assim, desde que o traço seja um operador contínuo de

$$
\gamma: H^{1}(\Omega) \rightarrow L^{r}(\partial \Omega), \quad \gamma: H^{1}(\Omega) \rightarrow L^{p \sigma_{g}}(\partial \Omega) \quad \text { e } \quad \gamma: H^{1}(\Omega) \rightarrow L^{q}(\partial \Omega),
$$

as demonstrações seguem análogas as demonstrações na Seção 3.2.

Entretanto, como comentamos anteriormente, não encontramos a hipótese (H1*) da forma como colocamos aqui. Na prática, quando já sabemos que as soluções dos problemas estudados são limitadas em $L^{\infty}(\Omega)$, o que é comum é supor que as funções $f$ e $g$ são limitadas com derivadas limitadas. 
APÊNDICE A 


\section{Referências Bibliográficas}

Adams(1975) Robert Adams. Sobolev space. Academic Press - Columbia University, New York. Citado na pág. $8,12,13,14$

Amann(1995) Herbet Amann. Linear and quasilinear parabolic problems. Abstract linear theory. Birkhäuser Verlag, Basel, Boston, Berlin. Citado na pág. 1, 5, 18, 19, 20, 21, 48

Aragao e Bezerra(2017) Gleiciane S. Aragao e Flank D. M. Bezerra. Upper semicontinuity of the pullback attractors of non-autonomous damped wave equations with terms concentrating on the boundary. To appear in Journal of Mathematical Analysis and Applicantions. Citado na pág. 3, 69

Aragao e Bruschi(2015) Gleiciane S. Aragao e Simone M. Bruschi. Limit of a nonlinear elliptic equation with concentrated terms and varying domains: the non uniformly lipschitz case. Eletronic Journal of Differential Equations, 2015:1-14. Citado na pág. 2

Aragao e Bruschi(2016) Gleiciane S. Aragao e Simone M. Bruschi. Concentrated terms and varing domains in elliptic equations: Lipschitz case. Mathematical Methods in the Applied Sciences, 39:3450-3460. Citado na pág. 2

Aragao e Oliva(2011) Gleiciane S. Aragao e Sergio M. Oliva. Asymptotic behavior of a reactiondiffusion problem with delay and reaction term concentrated in the boundary. Journal of Mathematical Sciences, 5:347-376. Citado na pág. 2

Aragao e Oliva(2012) Gleiciane S. Aragao e Sergio M. Oliva. Delay nonlinear boundary condition as limit of reactions concentrating in the boundary. Journal of Mathematical Sciences, 253:25732592. Citado na pág. 2

Aragao et al.(2012) Gleiciane S. Aragao, Antônio L. Pereira e Marcone C. Pereira. A nonlinear elliptic problem with terms concentrating in the boundary. Mathematical Methods in the Applied Sciences, 35:1110-1116. Citado na pág. 2

Aragao et al.(2014) Gleiciane S. Aragao, Antonio L. Pereira e Marcone C. Pereira. Attractors for a nonlinear parabolic problem with terms concentrating on the boundary. Journal of Dynamics and Differential Equations, 26:871-888. Citado na pág. 2

Arrieta e Carvalho(1999) José M. Arrieta e Alexandre N. Carvalho. Parabolic problems with nonlinear boundary conditions and critical nonlinearities. Journal of Differential Equations, 156: 376-506. Citado na pág. 2, 18, 31, 32, 33, 36

Arrieta et al.(2000) José M. Arrieta, Alexandre N. Carvalho e Aníbal Rodríguez-Bernal. Attractors of parabolic problems with nonlinear boundary conditions. uniform bounds. Communications in Partial Differential Equations, 25:1-37. Citado na pág. 2, 48

Arrieta et al.(2008) José M. Arrieta, Aníbal Rodríguez-Bernal e Angela Jiménes-Casas. Flux terms and robin boundary conditions as limit of reaction and potentials concentrating at the boundary. Revista Matemática Iberoamericana, 24:183-211. Citado na pág. 2, 3, 6, 24, 25, 26, 28, 29, 48, 58 
Brezis(2010) Haim Brezis. Functional analisys, sobolev spaces and partial differential equations. Springer Verlag, New York, Dordrecht, Heidelberg, London. Citado na pág. 5, 7, 8, 15, 18

Carvalho(2012) Alexandre N. Carvalho. Sistemas dinâmicos não lineares, 2012. Citado na pág. 1, 2, $5,22,23,24$

Carvalho(2001) Alexandre N. Carvalho. Equações semilineares parabólicas, 2001. Citado na pág. 1, $5,15,16,17,18,21,22$

Carvalho et al.(1997) Alexandre N. Carvalho, Sérgio M. Oliva, Antônio L. Pereira e Aníbal Rodríguez-Bernal. Attractors for parabolic problems with nonlinear boundary conditions. Journal of Mathematical Analysis and Applications, 207:409-461. Citado na pág. 2, 5, 20, 21, 31, 33, 37, 69

Czaja(2002) Radoslaw Czaja. Differential equations with sectorial operator. Springer-Verlag. Citado na pág. $1,5,20,33$

Hale(1988) Jack K. Hale. Asymptotic behavior of dissipative systems. American Mathematical Society, Rhode Island. Citado na pág. 1, 5, 22, 23

Haroske e Triebel(2008) Dorothee D. Haroske e Hans Triebel. Distribuitions, sobolev spaces, elliptic equations. EMS, Germany. Citado na pág. 1, 8, 11

Henry(1981) Daniel Henry. Geometric theory of semilinear parabolic equations. Springer-Verlag, Berlin, Heidelberg, New York. Citado na pág. 1, 5, 15, 18, 20, 21, 31, 33

Jiménes-Casas e Rodríguez-Bernal(2009) Ángela Jiménes-Casas e Aníbal Rodríguez-Bernal. Asymptotic behavior of a parabolic problem with terms concentrated in the boundary. Nonlinear Analysis Theory, Methods and Applications, 71:2377-2383. Citado na pág. 2, 3, 31, 36, 58, 67

Jiménes-Casas e Rodríguez-Bernal(2011) Ángela Jiménes-Casas e Aníbal Rodríguez-Bernal. Singular limit for a nonlinear parabolic equation with terms concentrating on the boundary. Journal of Mathematical Analysis and Applications, 379:567-588. Citado na pág. 2, 3, 24, 27, 31, $33,34,43,44,50,53,58,67$

Nogueira(2017) Ariadne Nogueira. Integrais concentradas na fronteira e aplicaçôes para problemas elipticos semilineares. Tese de Doutorado, Instituto de Matemática e Estátistica da Universidade de São Paulo. Citado na pág. 2

Oliva e Pereira(2002) Sergio M. Oliva e Antônio L. Pereira. Attractors for parabolic problems with nonlinear boundary conditions in fractional power space. Dynamics of Continuous, Discrete and Impulsive System 9. Citado na pág. 2, 5, 31

Rodríguez-Bernal(2011) Aníbal Rodríguez-Bernal. A singular pertubation in a linear parabolic equation with terms concentrating on the boundary. Revista Matemática Complutense, 25:165197. Citado na pág. $2,33,43,44,50$

Temam(1988) Roger Temam. Infinite dimensional dynamical systems in mechanics and physics. Springer-Verlag, New York. Citado na pág. 1, 5, 22

Triebel(1978) Hans Triebel. Interpolation theory, function spaces, differential operators. NH Publishing Company, Amsterdan, New York, Oxford. Citado na pág. 1, 5, 8, 14, 15, 18, 19

Trudinger(1967) Neil S. Trudinger. On imbeddings into orlicz spaces and some applications. Journal of Mathematics and Mechanics, 17. Citado na pág. 37

Yagi(2010) Atsushi Yagi. Abstract parabolic evolution equations and their applications. Springer Verlag, Heidelberg, Dordrecht, London, New York. Citado na pág. 1, 5, 8, 12, 14

Yosida(1980) Kosaku Yosida. Functional analisys. Springe Verlag, Berlin, Heidelberg, New York. Citado na pág. $1,5,7,15$ 